\title{
Interactions of bromine, chlorine, and iodine photochemistry during ozone depletions in Barrow, Alaska
}

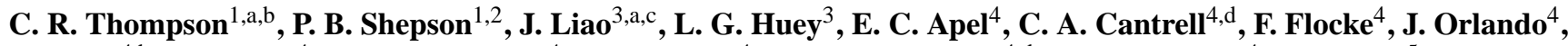 \\ A. Fried ${ }^{4, b}$, S. R. Hall ${ }^{4}$, R. S. Hornbrook ${ }^{4}$, D. J. Knapp ${ }^{4}$, R. L. Mauldin III ${ }^{4, d}$, D. D. Montzka ${ }^{4}$, B. C. Sive ${ }^{5, \mathrm{e}}$, \\ K. Ullmann ${ }^{4}$, P. Weibring ${ }^{4}$, and A. Weinheimer ${ }^{4}$ \\ ${ }^{1}$ Department of Chemistry, Purdue University, West Lafayette, Indiana, USA \\ ${ }^{2}$ Department of Earth, Atmospheric, and Planetary Sciences and Purdue Climate Change Research Center, \\ Purdue University, West Lafayette, Indiana, USA \\ ${ }^{3}$ School of Earth and Atmospheric Sciences, Georgia Institute of Technology, Atlanta, Georgia, USA \\ ${ }^{4}$ National Center for Atmospheric Research, Boulder, Colorado, USA \\ ${ }^{5}$ Institute for the Study of Earth, Oceans, and Space, University of New Hampshire, Durham, New Hampshire, USA \\ anow at: Chemical Sciences Division, Earth System Research Laboratory, NOAA, Boulder, Colorado, USA \\ ${ }^{b}$ now at: Institute of Arctic and Alpine Research, University of Colorado Boulder, Boulder, Colorado, USA \\ ${ }^{c}$ now at: Cooperative Institute for Research in Environmental Sciences (CIRES), University of Colorado Boulder, \\ Colorado, USA \\ ${ }^{\mathrm{d}}$ now at: Atmospheric and Ocean Sciences, University of Colorado Boulder, Boulder, Colorado, USA \\ ${ }^{e}$ now at: National Park Service, Lakewood, Colorado, USA
}

Correspondence to: C. R. Thompson (chelsea.thompson@noaa.gov)

Received: 30 July 2014 - Published in Atmos. Chem. Phys. Discuss.: 19 November 2014

Revised: 7 July 2015 - Accepted: 21 July 2015 - Published: 28 August 2015

\begin{abstract}
The springtime depletion of tropospheric ozone in the Arctic is known to be caused by active halogen photochemistry resulting from halogen atom precursors emitted from snow, ice, or aerosol surfaces. The role of bromine in driving ozone depletion events (ODEs) has been generally accepted, but much less is known about the role of chlorine radicals in ozone depletion chemistry. While the potential impact of iodine in the High Arctic is more uncertain, there have been indications of active iodine chemistry through observed enhancements in filterable iodide, probable detection of tropospheric IO, and recently, observation of snowpack photochemical production of $\mathrm{I}_{2}$. Despite decades of research, significant uncertainty remains regarding the chemical mechanisms associated with the bromine-catalyzed depletion of ozone, as well as the complex interactions that occur in the polar boundary layer due to halogen chemistry. To investigate this, we developed a zero-dimensional photochemical model, constrained with measurements from the 2009 OASIS field campaign in Barrow, Alaska. We simulated a 7-day period during late March that included a full ozone depletion
\end{abstract}

event lasting 3 days and subsequent ozone recovery to study the interactions of halogen radicals under these different conditions. In addition, the effects of iodine added to our Base Model were investigated. While bromine atoms were primarily responsible for ODEs, chlorine and iodine were found to enhance the depletion rates and iodine was found to be more efficient per atom at depleting ozone than $\mathrm{Br}$. The interaction between chlorine and bromine is complex, as the presence of chlorine can increase the recycling and production of $\mathrm{Br}$ atoms, while also increasing reactive bromine sinks under certain conditions. Chlorine chemistry was also found to have significant impacts on both $\mathrm{HO}_{2}$ and $\mathrm{RO}_{2}$, with organic compounds serving as the primary reaction partner for $\mathrm{Cl}$ atoms. The results of this work highlight the need for future studies on the production mechanisms of $\mathrm{Br}_{2}$ and $\mathrm{Cl}_{2}$, as well as on the potential impact of iodine in the High Arctic. 


\section{Introduction}

The importance of halogen chemistry in polar regions has been well established over the past few decades since the observation of near-surface boundary layer ozone depletion in the 1980s (Oltmans and Komhyr, 1986; Barrie et al., 1988; Bottenheim et al., 1990). Since that time, ozone depletion events (ODEs) have been observed at numerous Arctic and Antarctic locations (e.g., Bottenheim et al., 2002; Saiz-Lopez et al., 2007b; Simpson et al., 2007; Oltmans et al., 2012). ODEs are characterized by episodic depletions of tropospheric ozone from background mole ratios of approximately 35 parts per billion by volume (ppbv) to less than 2 ppbv over periods of hours to days. These events are known to occur following the onset of polar sunrise, and continue through polar spring, when temperatures are low and snow and sea ice are still present, with a stable atmospheric boundary layer (Simpson et al., 2007).

Photochemical reactions involving halogen radicals, notably bromine, are thought to be the primary cause of ODEs (see Simpson et al., 2007, for a review). The chemical destruction of $\mathrm{O}_{3}$ by $\mathrm{Br}$ can be described by Reactions (R1)(R3) (Platt and Hönninger, 2003).

$$
\begin{array}{r}
\mathrm{Br}_{2}+h v \rightarrow 2 \mathrm{Br} \\
\mathrm{Br}+\mathrm{O}_{3} \rightarrow \mathrm{BrO}+\mathrm{O}_{2} \\
\mathrm{BrO}+\mathrm{BrO} \rightarrow \mathrm{Br}_{2}+\mathrm{O}_{2}
\end{array}
$$

The efficiency of $\mathrm{Br}$ atoms in destroying ozone is due primarily to its relative lack of atmospheric sinks, and thus its relatively high gas-phase concentration, as well as its ability to recycle and regenerate from temporary sink species. Bromine atoms do not react appreciably with methane or other saturated hydrocarbons; thus, its primary sinks (other than $\mathrm{O}_{3}$ ) consist of a few oxygenated volatile organic compounds (OVOCs) (e.g., aldehydes) or unsaturated hydrocarbons, which result in production of $\mathrm{HBr}$, and $\mathrm{HO}_{x}$ species. $\mathrm{BrO}$ can react to regenerate a $\mathrm{Br}$ atom (via Reaction $\mathrm{R} 3$, followed by Reaction R1), or react with $\mathrm{HO}_{2}$ to produce $\mathrm{HOBr}$, which can in turn lead to the production of two $\mathrm{Br}$ atoms through a heterogeneous reaction mechanism termed the bromine explosion (Tang and McConnell, 1996; Vogt et al., 1996), as shown below.

$$
\begin{array}{r}
\mathrm{BrO}+\mathrm{HO}_{2} \rightarrow \mathrm{HOBr}+\mathrm{O}_{2} \\
\mathrm{HOBr}_{(\mathrm{g})} \rightarrow \mathrm{HOBr}_{(\mathrm{aq})} \\
\mathrm{HOBr}_{(\mathrm{aq})}+\mathrm{Br}_{(\mathrm{aq})}^{-}+\mathrm{H}_{(\mathrm{aq})}^{+} \rightarrow \mathrm{Br}_{2(\mathrm{aq})}+\mathrm{H}_{2} \mathrm{O} \\
\mathrm{Br}_{2(\mathrm{aq})} \rightarrow \mathrm{Br}_{2(\mathrm{~g})}
\end{array}
$$

The production of $\mathrm{Br}_{2}$ can thus be sustained on saline snow, ice, and aerosol surfaces, as has been confirmed in laboratory studies that have observed production of $\mathrm{Br}_{2}$ and $\mathrm{BrCl}$ from aqueous and frozen halide surfaces exposed to HOBr (Fickert et al., 1999; Adams et al., 2002; Huff and Abbatt, 2002), as well as in a recent field-based study that observed $\mathrm{Br}_{2}$ production from sunlit snowpacks in Barrow, Alaska (Pratt et al., 2013).

The presence of chlorine chemistry in the Arctic has been well recognized through indirect measurements of hydrocarbons (Jobson et al., 1994; Ariya et al., 1998; Keil and Shepson, 2006; Tackett et al., 2007) and through detection of photolyzable chlorine species (defined as $\left.\left[\mathrm{Cl}_{2}+\mathrm{HOCl}\right]\right)(\mathrm{Im}-$ pey et al., 1997); however, few direct measurements of chlorine species have been reported. The only currently reported measurements of ClO were by Tuckermann et al. (1997), who detected $\mathrm{ClO}$ at Spitsbergen. Unlike bromine, chlorine radicals efficiently oxidize a wide range of pollutants and volatile organic compounds (VOCs), often with faster rate coefficients than analogous reactions by the hydroxyl radical $(\mathrm{OH})$; thus, chlorine has an abundance of atmospheric sinks. Estimates of polar region $\mathrm{Cl}$ atom concentrations using hydrocarbon decay methods are typically in the range of $10^{4}$ $10^{5}$ molecules $\mathrm{cm}^{-3}$ (Jobson et al., 1994; Ariya et al., 1998; Boudries and Bottenheim, 2000), approximately 2-3 orders of magnitude lower than analogous estimates of $\mathrm{Br}$ (Cavender et al., 2008).

Like bromine, chlorine can react directly with $\mathrm{O}_{3}$, generating a $\mathrm{ClO}$ radical via Reaction (R8). The presence of $\mathrm{ClO}$ may also promote bromine-induced depletion of $\mathrm{O}_{3}$ through the fast cross-reaction of $\mathrm{BrO}$ and $\mathrm{ClO}$ that serves to regenerate $\mathrm{Br}$ atoms (Reaction R9) (Le Bras and Platt, 1995; Platt and Hönninger, 2003).

$$
\begin{array}{r}
\mathrm{Cl}+\mathrm{O}_{3} \rightarrow \mathrm{ClO}+\mathrm{O}_{2} \\
\mathrm{BrO}+\mathrm{ClO} \rightarrow \mathrm{Br}+\mathrm{OClO}\left(\text { or } \mathrm{Cl}+\mathrm{O}_{2}\right)
\end{array}
$$

Due to analytical challenges, few tropospheric observations of $\mathrm{Cl}_{2}$ and $\mathrm{ClO}$ exist; therefore, the role of $\mathrm{Cl}$ in ozone depletion events remains uncertain and has been much debated. Typical estimated $\mathrm{Cl}$ concentrations are likely too low for chlorine to be a significant direct contributor to ozone depletion. However, elevated levels of $\mathrm{Cl}_{2}$ (exceeding $100 \mathrm{pptv}$ ) were recently observed during the Ocean-Atmosphere-Sea Ice-Snowpack (OASIS) 2009 campaign in Barrow, Alaska, using chemical ionization mass spectrometry (CIMS) (Liao et al., 2014). The impact that such high levels of $\mathrm{Cl}_{2}$ could have on ODEs or on the oxidation chemistry of the Arctic troposphere has not been fully investigated. Although Reaction (R9) can act to enhance the rate of ozone depletion by propagating the $\mathrm{Br}$ cycle, formaldehyde, propanal, acetaldehyde and $\mathrm{HO}_{2}$ are all produced as by-products of VOC oxidation by $\mathrm{Cl}$ and are efficient sinks for $\mathrm{Br}$ or $\mathrm{BrO}$ radicals (Shepson et al., 1996; Sumner et al., 2002). Therefore, it is likely that the interaction between chlorine and bromine is complex. Modeling studies simulating ozone depletion events often use $\mathrm{BrCl}$ as the primary source of $\mathrm{Cl}$ atoms (Calvert and Lindberg, 2003; Piot and Von Glasow, 2008), and thus $[\mathrm{Cl}]$ is quite low and often insignificant. Few have added significant $\mathrm{Cl}_{2}$ sources (Sander et al., 1997; Piot and von Glasow, 2009). As a result, our understanding of chlorine chemistry in the Arctic is limited. 
Iodine may play a significant role in the photochemical reactions leading to ODEs (Sander et al., 1997; SaizLopez et al., 2007b, 2008; Mahajan et al., 2010) based on recent ground-based and satellite observations of IO of up to 20-50 pptv over snow- and ice-covered surfaces in coastal Antarctica (Saiz-Lopez et al., 2007a, b; Schönhardt et al., 2008; Frieß et al., 2010). Although IO has been routinely detected at high levels in Antarctica, there has been no successful set of IO measurements in the High Arctic to date. However, this is possibly due to limitations of current analytical methods rather than an absolute absence of iodine chemistry. Several studies in the Arctic have indicated the presence of iodine species through measurements of springtime peaks in filterable iodine (Sturges and Barrie, 1988; Barrie et al., 1994; Sirois and Barrie, 1999) and total gaseous iodine (Martinez et al., 1999). Hönninger (2002) was able to detect IO during only one instance above the detection limit at Alert in 2000 using long path DOAS, corresponding to $0.7 \mathrm{pptv}$ of IO if averaged over the $10 \mathrm{~km}$ light path. Mahajan et al. (2010) also observed up to $3.4 \mathrm{pptv}$ IO at the sub-Arctic location of Kujjuarapik, Canada. Most recently, $\mathrm{I}_{2}$ at levels $\leq 0.5 \mathrm{pptv}$ has been observed by our research group at Barrow, Alaska, by CIMS, lending direct evidence supporting the presence of at least low levels of iodine chemistry in the Arctic (Raso et al., 2015).

Due to the lack of iodine observations in the Arctic, models often omit iodine chemistry when simulating ODEs. However, this may significantly underestimate the rate of ozone depletion because the $\mathrm{BrO}+\mathrm{IO}$ cross-reaction (Reaction $\mathrm{R} 10$ ), which propagates the $\mathrm{Br}$ chain, has a rate constant that is approximately 2 orders of magnitude faster than the BrO self-reaction.

$\mathrm{BrO}+\mathrm{IO} \rightarrow \mathrm{Br}+\mathrm{I}+\mathrm{O}_{2}$

Indeed, previous modeling studies have found that significant enhancements in ozone depletion result from even small concentrations of reactive iodine (Calvert and Lindberg, 2004a; Saiz-Lopez et al., 2008; Mahajan et al., 2010).

Besides causing tropospheric ODEs, halogens can also impact $\mathrm{HO}_{x}$ and $\mathrm{NO}_{x}$ chemical cycles. The presence of reactive halogen species has the general effect of shifting the $\left[\mathrm{HO}_{2}\right] /[\mathrm{OH}]$ ratio towards $\mathrm{OH}$ (Platt and Hönninger, 2003; Thomas et al., 2012), primarily through Reactions (R11) and (R12), where $X$ can be $\mathrm{Br}, \mathrm{Cl}$, or I.

$$
\begin{array}{r}
X \mathrm{O}+\mathrm{HO}_{2} \rightarrow \mathrm{HO} X+\mathrm{O}_{2} \\
\mathrm{HO} X+h v \rightarrow X+\mathrm{OH}
\end{array}
$$

Bromine and chlorine atoms can react with formaldehyde (and higher molecular-weight aldehydes), leading to production of hydrogen halides and $\mathrm{HO}_{x}$. Alternatively, halogen atom reaction with $\mathrm{HO}_{2}$ can act as a $\mathrm{HO}_{x}$ sink. The inclusion of chlorine chemistry further impacts the $\mathrm{HO}_{x}$ chemistry because $\mathrm{RO}_{2}, \mathrm{HO}_{2}$, and carbonyl compounds are produced as side-products of $\mathrm{Cl}$ oxidation of VOCs (e.g., Reactions 13 $15)$.

$$
\begin{array}{r}
\mathrm{Cl}+\mathrm{CH}_{4}\left(+\mathrm{O}_{2}\right) \rightarrow \mathrm{CH}_{3} \mathrm{OO}^{\bullet}+\mathrm{HCl} \\
\mathrm{CH}_{3} \mathrm{OO}^{\bullet}+\mathrm{NO} \rightarrow \mathrm{CH}_{3} \mathrm{O}^{\bullet}+\mathrm{NO}_{2} \\
\mathrm{CH}_{3} \mathrm{O}^{\bullet}+\mathrm{O}_{2} \rightarrow \mathrm{HCHO}+\mathrm{HO}_{2}
\end{array}
$$

Halogen chemistry also generally increases the Leighton ratio $\left(\left[\mathrm{NO}_{2}\right] /[\mathrm{NO}]\right)$ through conversion of $\mathrm{NO}$ to $\mathrm{NO}_{2}$ via $\mathrm{Re}$ actions (R14) and (R16) (Platt and Hönninger, 2003), where $X$ is $\mathrm{Br}, \mathrm{Cl}$, or $\mathrm{I}$. $X$ or $X \mathrm{O}$ can also react with $\mathrm{NO}_{2}$ to form $X \mathrm{NO}_{2}$ or $X \mathrm{ONO}_{2}$, respectively (Reactions R17-R18); however, the net effect of halogen chemistry is an increase in the $\left[\mathrm{NO}_{2}\right] /[\mathrm{NO}]$ ratio.

$$
\begin{array}{r}
X \mathrm{O}+\mathrm{NO} \rightarrow X+\mathrm{NO}_{2} \\
X+\mathrm{NO}_{2} \rightarrow X \mathrm{NO}_{2} \\
X \mathrm{O}+\mathrm{NO}_{2} \rightarrow X \mathrm{ONO}_{2}
\end{array}
$$

In this work, we utilize a zero-dimensional model to further investigate the chemical interactions occurring in the Arctic related to the chemistry of halogen radicals and the interactions between bromine, chlorine, and iodine. The OASIS 2009 campaign, conducted in March and April in Barrow, Alaska, provides a valuable opportunity to perform a unique study of halogen chemistry using direct observations of a variety of atmospheric species, including $\mathrm{HO}_{x}$ and $X_{\mathrm{O}}$ radicals, and molecular halogens, with high time resolution measured concurrently and from a single location. During OASIS, measurements of a large suite of saturated, unsaturated, and oxygenated VOCs, numerous halogenated species (e.g., $\mathrm{Br}_{2}, \mathrm{Cl}_{2}, \mathrm{BrCl}, \mathrm{HOBr}, \mathrm{BrO}, \mathrm{ClO}$ ), $\mathrm{OH}, \mathrm{HO}_{2}, \mathrm{NO}, \mathrm{NO}_{2}$, $\mathrm{HONO}, \mathrm{O}_{3}$, and actinic radiation were conducted. We constrain our model with time-resolved data from OASIS, in order to investigate the following questions.

- What is the fraction of ozone depleted by each halogen atom, and how does each of the halogens impact the rate and timescale of ozone depletion?

- How do chlorine and iodine impact bromine chemistry relating to ODEs?

- What is the effect of halogen species on $\mathrm{HO}_{x}$ and $\mathrm{NO}_{x}$ chemistry, and, conversely, what is the effect of $\mathrm{HO}_{x}$ and $\mathrm{NO}_{x}$ on halogen chemistry?

- What is the importance of chlorine chemistry (e.g., is it only the $\mathrm{BrO}+\mathrm{ClO}$ cross-reaction that makes chlorine chemistry important)?

Several modeling scenarios were constructed in an effort to address these questions and to dissect the complex chemistry occurring during ODEs. The results of this work have been organized in the following manner.

- Section 3.1: comparison of modeled vs. observed mole ratios for pertinent species 
- Section 3.2: contribution of $\mathrm{Br}, \mathrm{Cl}$, and I to ozone depletion and the rate and timescale of ozone loss

- Section 3.3: impact of chlorine on bromine chemistry and oxidation capacity

- Section 3.4: impact of iodine and bromine-iodine interactions

\section{Methods}

\subsection{Field campaign and measurements description}

The OASIS (Ocean-Atmosphere-Sea Ice-Snowpack) 2009 campaign occurred during the months of February through April as part of the most recent International Polar Year (2007-2009). The motivation for OASIS was to investigate the chemical and physical processes leading to ozone and mercury depletion events in polar spring. The OASIS campaign represents the largest suite of simultaneous and colocated atmospheric trace gas measurements conducted in the Arctic to date, and as such, has provided a rich data set for understanding chemical processes in the Arctic troposphere.

The atmospheric measurements employed in this work were conducted from mobile instrument trailers located near the Barrow Arctic Research Consortium (BARC) facility on the Naval Arctic Research Laboratory (NARL) campus. Winds arriving at the measurement site are primarily northeasterly (i.e., from the sea ice), and, thus, should represent background conditions with influence from only natural processes and snow-ice interactions. Occasional winds from the west (i.e., from the town of Barrow) are easily identifiable by coincident enhancements in both $\mathrm{NO}_{x}$ and $\mathrm{CO}$.

Table 5 lists the measurements conducted at OASIS that were used in this modeling study. This is not an exhaustive list of all measurements performed during the campaign. As the methods have all been described previously, they will only be described very briefly here. Ozone, NO, and $\mathrm{NO}_{2}$ were measured using the well-established chemiluminescence technique as described in Ridley et al. (1992) and Ryerson et al. (2000). $\mathrm{O}_{3}$ and $\mathrm{NO}_{x}$ were measured at three heights $(2,6$, and $18 \mathrm{ft})$ from a fixed tower. The data from the $6 \mathrm{ft}$ height were used in this analysis. Carbon monoxide $(\mathrm{CO})$ concentrations were measured using a standard commercial CO analyzer (Thermo Scientific) with infrared absorption detection. The inorganic halogen species $\mathrm{Br}_{2}, \mathrm{Cl}_{2}, \mathrm{BrO}$, and $\mathrm{HOBr}$ were measured in situ at a height of $\sim 1.5 \mathrm{~m}$ above the snowpack with a chemical ionization mass spectrometer (CIMS) employing $\mathrm{I}^{-}$ion chemistry. This method, as used in Barrow, and these observations have been published in Liao et al. (2011, 2012, 2014). ClO was measured at a height of $\sim 3 \mathrm{~m}$ above the snowpack via chemical conversion to a stable, halogenated product followed by online separation and detection by gas chromatography with electron capture detection (Stephens et al., 2012). Formalde-
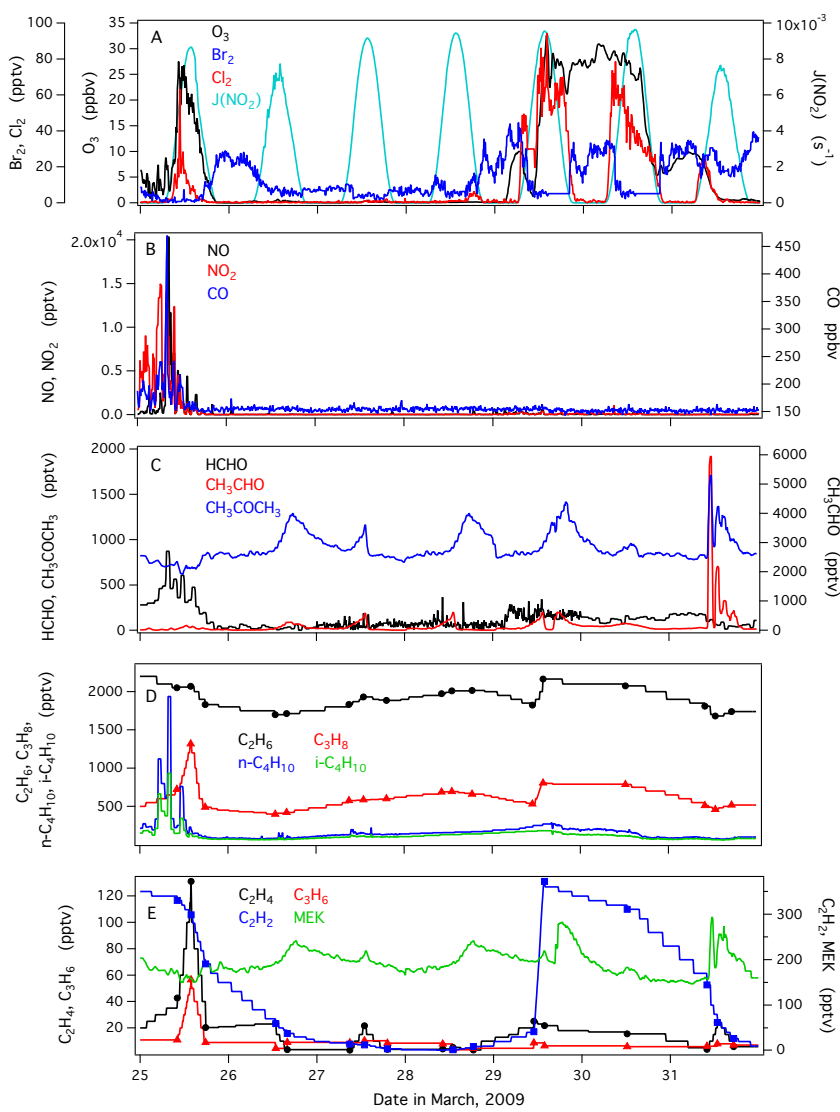

Figure 1. Ambient measurements of $\mathrm{O}_{3}, \mathrm{Cl}_{2}, \mathrm{Br}_{2}, \mathrm{NO}_{2}, \mathrm{NO}, \mathrm{CO}$, VOCs, and OVOCs that are incorporated into the model. Mixing ratios for the NMHCs that were collected as canister samples were interpolated between samples. Available canister sample data points are indicated on their respective plot. The photolysis rate coefficient $(J)$ is shown for $\mathrm{NO}_{2}$ as an example in Panel (a). Time is expressed in Alaska Standard Time.

hyde $(\mathrm{HCHO})$ concentrations were measured at $1 \mathrm{~Hz}$ frequency from the fixed tower using a tunable diode laser absorption spectrometer as described in Fried et al. (1997) and Lancaster et al. (2000). As with the $\mathrm{O}_{3}$ and $\mathrm{NO}_{x}$ measurements, only data at $6 \mathrm{ft}$ were used in this analysis. HONO was measured using the Long-Path Absorption Photometer (LOPAP) technique described in Villena et al. (2011) with a reported $10 \%$ measurement uncertainty. Finally, a large suite of organic compounds was measured both in situ by fast GCMS (Apel et al., 2010) and via whole air canister samples with offline GC-MS (Russo et al., 2010). Those species that were directly used in this analysis are listed in Table 5.

\subsection{Model description}

The model used for this study is a zero-dimensional, photochemical model solved using the FACSIMILE commercial software. It incorporates much of the known gas-phase chemistry that occurs in the Arctic in 220 gas-phase reactions and 
42 photolysis reactions (Tables 1 and 2). The model also includes deposition/uptake to snow/aerosol surfaces for certain species, aqueous-phase halide reactions (Tables 3 and 4), and a simple dilution rate for long-lived species (e.g., PAN).

The model was developed to investigate, as a particularly useful example case study, the period 25 through 31 March 2009, which included a 3-day ozone depletion event during which $\mathrm{O}_{3}$ remained below 1 ppbv. This time period was chosen to investigate the changes in halogen interactions under different ozone regimes, i.e., background ozone ( $\sim 30-35 \mathrm{ppbv}$ ), ozone depleting periods, full ozone depletion $\left(\mathrm{O}_{3}<5 \mathrm{ppbv}\right)$, and ozone recovery, during which winds were consistently (with the exception of the first half of 25 March) arriving at the measurement site directly from the frozen Arctic Ocean. To enable focused pursuit of specific questions about gas-phase free radical chemistry, the model was constrained to observed values of $\mathrm{O}_{3}, \mathrm{C}_{2} \mathrm{H}_{2}, \mathrm{C}_{2} \mathrm{H}_{4}$, $\mathrm{C}_{2} \mathrm{H}_{6}, \mathrm{C}_{3} \mathrm{H}_{8}, \mathrm{C}_{3} \mathrm{H}_{6}, n-\mathrm{C}_{4} \mathrm{H}_{10}, i-\mathrm{C}_{4} \mathrm{H}_{10}, \mathrm{HCHO}, \mathrm{CH}_{3} \mathrm{CHO}$, $\mathrm{CH}_{3} \mathrm{COCH}_{3}$, methyl ethyl ketone, $\mathrm{Cl}_{2}, \mathrm{Br}_{2}, \mathrm{NO}, \mathrm{NO}_{2}$, and $\mathrm{CO}$, as well as calculated time-varying photolysis rate coefficients $(J)$ for $\mathrm{O}_{3}$ and $\mathrm{NO}_{2}$. The in situ measurements that were used to constrain the model are presented in Fig. 1. $\mathrm{NO}_{2}$ mole ratios in Barrow are often quite variable and relatively high compared to other polar measurement locations (Beine et al., 2002). At times, winds travel from the southwesterly direction, bringing air influenced by Barrow emissions over the measurement site. This is the case for the high $\mathrm{NO}_{2} \mathrm{ob}-$ served at the beginning of 25 March, which correlates with enhanced CO. Observed values were incorporated at $10 \mathrm{~min}$ time steps, calculated as $10 \mathrm{~min}$ averages of the reported data, over the entire 7-day period. Constraining the model with these time-varying observations precludes the need for parameterization of atmospheric transport due to advection, and thus justifies the use of a zero-dimensional model for this study. It is important to note that, because our objective was to investigate the halogen chemistry occurring during this time period rather than to attempt to simulate an ozone depletion event, we have constrained our model with the observed ozone mole ratios in order to fully study the fast chemical interactions occurring under these observed conditions, which are strong functions of $\mathrm{O}_{3}$.

Mole ratios of $\mathrm{CH}_{4}$ were held constant at an average value for this time of $1.89 \mathrm{ppmv}$ as reported by the NOAA-ESRL Barrow Observatory. [ $\left.\mathrm{H}_{2} \mathrm{O}\right]$ was calculated for 25 March from observed meteorological conditions of $78 \%$ relative humidity and an ambient temperature of $-19.5^{\circ} \mathrm{C}$ (NOAA; Barrow airport data), corresponding to a water vapor concentration of $2.23 \times 10^{16}$ molecules $\mathrm{cm}^{-3}$. This was held constant throughout the simulation. Temperature was not varied in the model.

The gas-phase chemical reactions and corresponding rate constants used in the model are shown in Table 1. Unless otherwise noted, all rate constants were calculated for a temperature of $248 \mathrm{~K}$, consistent with average ambient conditions in Barrow for this time (NOAA Barrow Observatory).
This mechanism includes halogen, $\mathrm{HO}_{x}, \mathrm{NO}_{x}$, and VOC chemistry associated with ozone depletions in the Arctic spring. The inorganic iodine reaction scheme used here is adapted from McFiggans et al. (2000, 2002), Calvert and Lindberg (2004a, b), and Saiz-Lopez et al. (2008). Organic iodine compounds are not included. Although some organic iodine compounds have been observed in coastal and marine locations (Carpenter et al., 1999; Jones et al., 2010), $\mathrm{I}_{2}$ is likely the major source of atmospheric iodine (Saiz-Lopez and Plane, 2004) and, thus, was assumed to be the reactive I source in this model. It should also be noted that while I does not react appreciably with VOCs, it is likely that I and IO do react with $\mathrm{RO}_{2}$ radicals (Sommariva et al., 2012). However, these reactions are not included here. Thus, though the iodine reaction scheme agrees with previous studies, it is likely incomplete; therefore, modeled iodine chemistry should be taken only as potential impacts to help direct future research efforts.

Photodissociation reactions included in this model are listed in Table 2. For many of the species, time-varying $J$ coefficients were calculated using a modified version of the Tropospheric Ultraviolet and Visible (TUV) radiation model (Madronich and Flocke, 1999) based on in situ $0.1 \mathrm{~Hz}$ measurements of downwelling actinic flux conducted throughout the duration of the OASIS campaign and a surface albedo of 0.8. Upwelling flux was estimated as a function of solar zenith angle assuming clear sky conditions. The sum of upwelling and downwelling radiation was used by the TUV model to calculate the total photolysis frequencies. $J$ coefficients for solar noon on 25 March (listed as $J_{\max }$ ) are provided in Table 2 as an example. $J$ coefficients were input into the model at $10 \mathrm{~min}$ time steps for $\mathrm{O}_{3}$ and $\mathrm{NO}_{2}$. All other $J$ values were scaled to $J_{\mathrm{NO} 2}$ in the modeling code.

$J$ coefficients from OASIS were not available for $\mathrm{OClO}$, $\mathrm{HOCl}$, or iodine compounds. An estimate for $J_{\max }$ for $\mathrm{OClO}$ was taken from Pöhler et al. (2010) and that for $\mathrm{HOCl}$ was taken from an Arctic model study by Lehrer et al. (2004), which were then scaled to $J_{\mathrm{NO} 2}$. $J$ coefficients for iodine compounds were calculated according to the work of Calvert and Lindberg (2004a, b), who also simulated conditions for late March in Barrow, although we note that there is a larger uncertainty for the photolysis coefficients for the iodine species, with the exception of $\mathrm{I}_{2}$. Time-varying $J$ coefficients for the iodine species were calculated using a fourthorder polynomial and varying the solar zenith angle from 98.4 to $72.3^{\circ}$.

Deposition of species to the snowpack is estimated based on measured dry deposition velocities and applied using Eq. (1) to calculate transfer coefficients $\left(k_{\mathrm{t}}\right)$ from the gas to aqueous phase.

$k_{\mathrm{t}}=\frac{V_{\mathrm{d}}}{h}$

$V_{\mathrm{d}}$ is the dry deposition velocity (in $\mathrm{cm} \mathrm{s}^{-1}$ ) and $h$ is the boundary layer height. Most previous estimates of ozone de- 
Table 1. Gas-phase chemical reactions used in the model. All rate constants are calculated for a temperature of $248 \mathrm{~K}$ unless otherwise noted and are expressed in units of $\mathrm{cm}^{3}$ molecule ${ }^{-1} \mathrm{~s}^{-1}$.

\begin{tabular}{|c|c|c|}
\hline Reaction & Rate constant & Reference \\
\hline $\mathrm{O}\left({ }^{1} D\right)+\mathrm{M} \rightarrow \mathrm{O}\left({ }^{3} P\right)$ & $7.22 \times 10^{-11}$ & Dunlea and Ravishankara (2004) \\
\hline $\mathrm{O}\left({ }^{3} P\right)+\mathrm{O}_{2} \rightarrow \mathrm{O}_{3}$ & $2.12 \times 10^{-14}$ & Atkinson et al. (2004) \\
\hline $\mathrm{O}\left({ }^{1} D\right)+\mathrm{H}_{2} \mathrm{O} \rightarrow 2 \mathrm{OH}$ & $2.2 \times 10^{-10}$ & Atkinson et al. (2004) \\
\hline $\mathrm{OH}+\mathrm{O}_{3} \rightarrow \mathrm{HO}_{2}$ & $3.84 \times 10^{-14}$ & Atkinson et al. (2004) \\
\hline $\mathrm{OH}+\mathrm{HO}_{2} \rightarrow \mathrm{H}_{2} \mathrm{O}$ & $1.34 \times 10^{-10}$ & Atkinson et al. (2004) \\
\hline $\mathrm{OH}+\mathrm{H}_{2} \mathrm{O}_{2} \rightarrow \mathrm{HO}_{2}+\mathrm{H}_{2} \mathrm{O}$ & $1.52 \times 10^{-12}$ & Atkinson et al. (2004) \\
\hline $\left.\mathrm{OH}+\mathrm{O}^{3} P\right) \rightarrow \mathrm{O}_{2}$ & $3.74 \times 10^{-11}$ & Atkinson et al. (2004) \\
\hline $\mathrm{OH}+\mathrm{OH} \rightarrow \mathrm{H}_{2} \mathrm{O}+\mathrm{O}\left({ }^{3} P\right)$ & $1.74 \times 10^{-12}$ & Atkinson et al. (2004) \\
\hline $\mathrm{OH}+\mathrm{OH} \rightarrow \mathrm{H}_{2} \mathrm{O}_{2}$ & $1.86 \times 10^{-11}$ & Atkinson et al. (2004) \\
\hline $\mathrm{OH}+\mathrm{NO}_{3} \rightarrow \mathrm{HO}_{2}+\mathrm{NO}_{2}$ & $2.0 \times 10^{-11}$ & Atkinson et al. (2004) \\
\hline $\mathrm{HO}_{2}+\mathrm{NO}_{3} \rightarrow \mathrm{HNO}_{3}$ & $4.0 \times 10^{-12}$ & Atkinson et al. (2004) \\
\hline $\mathrm{HO}_{2}+\mathrm{O}_{3} \rightarrow \mathrm{OH}+2 \mathrm{O}_{2}$ & $1.39 \times 10^{-15}$ & Atkinson et al. (2004) \\
\hline $\mathrm{HO}_{2}+\mathrm{HO}_{2} \rightarrow \mathrm{H}_{2} \mathrm{O}_{2}+\mathrm{O}_{2}$ & $2.58 \times 10^{-12}$ & Atkinson et al. (2004) \\
\hline $\mathrm{NO}+\mathrm{OH} \rightarrow \mathrm{HONO}$ & $3.49 \times 10^{-11}$ & Atkinson et al. (2004) \\
\hline $\mathrm{NO}+\mathrm{HO}_{2} \rightarrow \mathrm{NO}_{2}+\mathrm{OH}$ & $9.59 \times 10^{-12}$ & Atkinson et al. (2004) \\
\hline $\mathrm{NO}+\mathrm{O}_{3} \rightarrow \mathrm{NO}_{2}$ & $7.09 \times 10^{-15}$ & Sander et al. (2006) \\
\hline $\mathrm{NO}+\mathrm{NO}_{3} \rightarrow \mathrm{NO}_{2}+\mathrm{NO}_{2}$ & $2.98 \times 10^{-11}$ & Sander et al. (2006) \\
\hline $\mathrm{NO}_{2}+\mathrm{OH} \rightarrow \mathrm{HNO}_{3}$ & $1.2 \times 10^{-10}$ & Atkinson et al. (2004) \\
\hline $\mathrm{NO}_{2}+\mathrm{HO}_{2} \longleftrightarrow \rightarrow \mathrm{HNO}_{4}$ & f: $8.6 \times 10^{-12}$ r: $1.32 \times 10^{-4}$ & Atkinson et al. (2004) \\
\hline $\mathrm{NO}_{2}+\mathrm{O}_{3} \rightarrow \mathrm{NO}_{3}$ & $6.15 \times 10^{-18}$ & Sander et al. (2006) \\
\hline $\mathrm{NO}_{2}+\mathrm{NO}_{3} \leftarrow \mathrm{N}_{2} \mathrm{O}_{5}$ & f: $1.83 \times 10^{-12}$ r: $3.76 \times 10^{-5}$ & Atkinson et al. (2004) \\
\hline $\mathrm{NO}_{2}+\mathrm{CH}_{3} \mathrm{COOO} \leftarrow \mathrm{PAN}$ & f: $1.4 \times 10^{-11}$ r: $3.1 \times 10^{-8}$ & Atkinson et al. (2004) \\
\hline $\mathrm{NO}_{3}+\mathrm{NO}_{3} \rightarrow \mathrm{NO}_{2}+\mathrm{NO}_{2}$ & $4.36 \times 10^{-17}$ & Sander et al. (2006) \\
\hline $\mathrm{N}_{2} \mathrm{O}_{5}+\mathrm{H}_{2} \mathrm{O} \rightarrow \mathrm{HNO}_{3}+\mathrm{HNO}_{3}$ & $2.6 \times 10^{-22}$ & Atkinson et al. (2004) \\
\hline $\mathrm{HONO}+\mathrm{OH} \rightarrow \mathrm{NO}_{2}+\mathrm{H}_{2} \mathrm{O}$ & $3.74 \times 10^{-12}$ & Sander et al. (2006) \\
\hline $\mathrm{HNO}_{3}+\mathrm{OH} \rightarrow \mathrm{NO}_{3}+\mathrm{H}_{2} \mathrm{O}$ & $1.5 \times 10^{-13}$ & Atkinson et al. (2004) \\
\hline $\mathrm{HNO}_{4}+\mathrm{OH} \rightarrow \mathrm{NO}_{2}+\mathrm{H}_{2} \mathrm{O}$ & $6.2 \times 10^{-12}$ & Atkinson et al. (2004) \\
\hline $\mathrm{CO}+\mathrm{OH} \rightarrow \mathrm{HO}_{2}+\mathrm{CO}_{2}$ & $2.4 \times 10^{-13}$ & Atkinson et al. (2004) \\
\hline $\mathrm{CH}_{4}+\mathrm{OH} \rightarrow \mathrm{CH}_{3} \mathrm{OO}+\mathrm{H}_{2} \mathrm{O}$ & $1.87 \times 10^{-15}$ & Sander et al. (2006) \\
\hline $\mathrm{C}_{2} \mathrm{H}_{2}+\mathrm{OH} \rightarrow \mathrm{C}_{2} \mathrm{H}_{2} \mathrm{OH}$ & $7.8 \times 10^{-13}$ & Atkinson et al. (2004) \\
\hline $\mathrm{C}_{2} \mathrm{H}_{6}+\mathrm{OH} \rightarrow \mathrm{C}_{2} \mathrm{H}_{5} \mathrm{OO}$ & $1.18 \times 10^{-13}$ & Lurmann et al. (1986) \\
\hline $\mathrm{C}_{2} \mathrm{H}_{4}+\mathrm{OH} \rightarrow \mathrm{C}_{2} \mathrm{H}_{4} \mathrm{OH}$ & $1.02 \times 10^{-11}$ & Vakhtin et al. (2003) \\
\hline $\mathrm{C}_{3} \mathrm{H}_{8}+\mathrm{OH} \rightarrow n \mathrm{C}_{3} \mathrm{H}_{7} \mathrm{O}_{2}$ & $1.56 \times 10^{-13}$ & Harris and Kerr (1988) \\
\hline $\mathrm{C}_{3} \mathrm{H}_{8}+\mathrm{OH} \rightarrow i \mathrm{C}_{3} \mathrm{H}_{7} \mathrm{O}_{2}$ & $6.64 \times 10^{-13}$ & Harris and Kerr (1988) \\
\hline $\mathrm{C}_{3} \mathrm{H}_{6}+\mathrm{OH} \rightarrow \mathrm{C}_{3} \mathrm{H}_{6} \mathrm{OH}$ & $3.63 \times 10^{-11}$ & Atkinson et al. (2004) \\
\hline $\mathrm{C}_{3} \mathrm{H}_{6} \mathrm{O}+\mathrm{OH} \rightarrow$ products & $2.51 \times 10^{-11}$ & Atkinson et al. (2004) \\
\hline$n \mathrm{C}_{3} \mathrm{H}_{7} \mathrm{O}_{2}+\mathrm{NO} \rightarrow \mathrm{NO}_{2}+\mathrm{C}_{3} \mathrm{H}_{6} \mathrm{O}+\mathrm{HO}_{2}$ & $5.4 \times 10^{-11}$ & Eberhard et al. (1996) \\
\hline$i \mathrm{C}_{3} \mathrm{H}_{7} \mathrm{O}_{2}+\mathrm{NO} \rightarrow \mathrm{NO}_{2}+\mathrm{CH}_{3} \mathrm{COCH}_{3}+\mathrm{HO}_{2}$ & $1.2 \times 10^{-11}$ & Eberhard and Howard (1996) \\
\hline$n \mathrm{C}_{4} \mathrm{H}_{10}+\mathrm{OH} \rightarrow n \mathrm{C}_{4} \mathrm{H}_{9} \mathrm{OO}$ & $1.64 \times 10^{-12}$ & Donahue et al. (1998) \\
\hline$i \mathrm{C}_{4} \mathrm{H}_{10}+\mathrm{OH} \rightarrow \mathrm{CH}_{3} \mathrm{COCH}_{3}+\mathrm{CH}_{3} \mathrm{OO}$ & $1.65 \times 10^{-12}$ & Donahue et al. (1998) \\
\hline$n \mathrm{C}_{4} \mathrm{H}_{9} \mathrm{OO}+\mathrm{NO} \rightarrow$ n-Butanal $+\mathrm{NO}_{2}+\mathrm{HO}_{2}$ & $5.4 \times 10^{-11}$ & Michalowski et al. (2000) \\
\hline$n \mathrm{C}_{4} \mathrm{H}_{9} \mathrm{OO}+\mathrm{CH}_{3} \mathrm{OO} \rightarrow$ n-Butanal $+\mathrm{HCHO}+\mathrm{HO}_{2}+\mathrm{HO}_{2}$ & $6.7 \times 10^{-13}$ & Michalowski et al. (2000) \\
\hline$n \mathrm{C}_{4} \mathrm{H}_{9} \mathrm{OO}+\mathrm{CH}_{3} \mathrm{OO} \rightarrow$ n-Butanal $+\mathrm{CH}_{3} \mathrm{OH}$ & $2.3 \times 10^{-13}$ & Michalowski et al. (2000) \\
\hline$n \mathrm{C}_{4} \mathrm{H}_{9} \mathrm{OO}+\mathrm{CH}_{3} \mathrm{OO} \rightarrow n \mathrm{C}_{4} \mathrm{H}_{9} \mathrm{OH}+\mathrm{HCHO}$ & $2.3 \times 10^{-13}$ & Michalowski et al. (2000) \\
\hline $\mathrm{CH}_{3} \mathrm{OH}+\mathrm{OH} \rightarrow \mathrm{CH}_{3} \mathrm{O}$ & $7.09 \times 10^{-13}$ & Atkinson et al. (2004) \\
\hline $\mathrm{n}$-Butanal $+\mathrm{OH} \rightarrow$ products & $2.0 \times 10^{-11}$ & Michalowski et al. (2000) \\
\hline $\mathrm{CH}_{3} \mathrm{OO}+\mathrm{HO}_{2} \rightarrow \mathrm{CH}_{3} \mathrm{OOH}$ & $8.82 \times 10^{-12}$ & Atkinson et al. (2004) \\
\hline $\mathrm{C}_{2} \mathrm{H}_{5} \mathrm{OO}+\mathrm{HO}_{2} \rightarrow \mathrm{C}_{2} \mathrm{H}_{5} \mathrm{OOH}$ & $1.12 \times 10^{-11}$ & Atkinson et al. (2004) \\
\hline $\mathrm{CH}_{3} \mathrm{COOO}+\mathrm{HO}_{2} \rightarrow \mathrm{CH}_{3} \mathrm{COOOH}$ & $2.54 \times 10^{-11}$ & DeMore et al. (1997) \\
\hline $\mathrm{C}_{2} \mathrm{H}_{5} \mathrm{OOH}+\mathrm{OH} \rightarrow \mathrm{C}_{2} \mathrm{H}_{5} \mathrm{OO}$ & $6.0 \times 10^{-12}$ & Atkinson et al. (2004) \\
\hline
\end{tabular}


Table 1. Continued.

\begin{tabular}{|c|c|c|}
\hline Reaction & Rate constant & Reference \\
\hline $\mathrm{CH}_{3} \mathrm{OO}+\mathrm{CH}_{3} \mathrm{OO} \rightarrow \mathrm{HCHO}+\mathrm{HO}_{2}$ & $3.64 \times 10^{-13}$ & Lurmann et al. (1986) \\
\hline $\mathrm{CH}_{3} \mathrm{OOH}+\mathrm{OH} \rightarrow \mathrm{HCHO}+\mathrm{H}_{2} \mathrm{O}+\mathrm{OH}$ & $2.54 \times 10^{-12}$ & Sander and Crutzen (1996) \\
\hline $\mathrm{CH}_{3} \mathrm{OOH}+\mathrm{OH} \rightarrow \mathrm{CH}_{3} \mathrm{OO}+\mathrm{H}_{2} \mathrm{O}$ & $6.01 \times 10^{-12}$ & Sander and Crutzen (1996) \\
\hline $\mathrm{CH}_{3} \mathrm{OO}+\mathrm{HO}_{2} \rightarrow \mathrm{CH}_{3} \mathrm{OOH}$ & $1.01 \times 10^{-11}$ & Atkinson et al. (2004) \\
\hline $\mathrm{CH}_{3} \mathrm{OO}+\mathrm{NO} \rightarrow \mathrm{HCHO}+\mathrm{HO}_{2}+\mathrm{NO}_{2}$ & $8.76 \times 10^{-12}$ & Atkinson et al. (2004) \\
\hline $\mathrm{CH}_{3} \mathrm{OO}+n \mathrm{C}_{3} \mathrm{H}_{7} \mathrm{O}_{2} \rightarrow \mathrm{HCHO}+\mathrm{C}_{3} \mathrm{H}_{6} \mathrm{O}+\mathrm{HO}_{2}+\mathrm{HO}_{2}$ & $6.70 \times 10^{-13}$ & Lightfoot et al. (1992) \\
\hline $\mathrm{CH}_{3} \mathrm{OO}+n \mathrm{C}_{3} \mathrm{H}_{7} \mathrm{O}_{2} \rightarrow \mathrm{C}_{3} \mathrm{H}_{6} \mathrm{O}+\mathrm{CH}_{3} \mathrm{OH}$ & $2.3 \times 10^{-13}$ & Lightfoot et al. (1992) \\
\hline $\mathrm{CH}_{3} \mathrm{OO}+n \mathrm{C}_{3} \mathrm{H}_{7} \mathrm{O}_{2} \rightarrow \mathrm{HCHO}+n \mathrm{C}_{3} \mathrm{H}_{7} \mathrm{OH}$ & $2.3 \times 10^{-13}$ & Lightfoot et al. (1992) \\
\hline $\mathrm{CH}_{3} \mathrm{OO}+i \mathrm{C}_{3} \mathrm{H}_{7} \mathrm{O}_{2} \rightarrow \mathrm{HCHO}+\mathrm{CH}_{3} \mathrm{COCH}_{3}+\mathrm{HO}_{2}+\mathrm{HO}_{2}$ & $1.2 \times 10^{-14}$ & Lightfoot et al. (1992) \\
\hline $\mathrm{CH}_{3} \mathrm{OO}+i \mathrm{C}_{3} \mathrm{H}_{7} \mathrm{O}_{2} \rightarrow \mathrm{CH}_{3} \mathrm{COCH}_{3}+\mathrm{CH}_{3} \mathrm{OH}$ & $4.1 \times 10^{-15}$ & Lightfoot et al. (1992) \\
\hline $\mathrm{CH}_{3} \mathrm{OO}+i \mathrm{C}_{3} \mathrm{H}_{7} \mathrm{O}_{2} \rightarrow \mathrm{HCHO}+i \mathrm{C}_{3} \mathrm{H}_{7} \mathrm{OH}$ & $4.1 \times 10^{-15}$ & Lightfoot et al. (1992) \\
\hline $\mathrm{CH}_{3} \mathrm{OO}+\mathrm{C}_{2} \mathrm{H}_{5} \mathrm{OO} \rightarrow \mathrm{CH}_{3} \mathrm{CHO}+\mathrm{HCHO}+\mathrm{HO}_{2}+\mathrm{HO}_{2}$ & $2.0 \times 10^{-13}$ & Kirchner and Stockwell (1996) \\
\hline $\mathrm{CH}_{3} \mathrm{OO}+\mathrm{CH}_{3} \mathrm{COOO} \rightarrow \mathrm{HCHO}+\mathrm{CH}_{3} \mathrm{OO}+\mathrm{HO}_{2}$ & $1.58 \times 10^{-11}$ & Kirchner and Stockwell (1996) \\
\hline $\mathrm{C}_{2} \mathrm{H}_{5} \mathrm{OO}+\mathrm{NO} \rightarrow \mathrm{CH}_{3} \mathrm{CHO}+\mathrm{HO}_{2}+\mathrm{NO}_{2}$ & $8.68 \times 10^{-12}$ & Lurmann et al. (1986) \\
\hline $\mathrm{C}_{2} \mathrm{H}_{5} \mathrm{OO}+\mathrm{HO}_{2} \rightarrow \mathrm{C}_{2} \mathrm{H}_{5} \mathrm{OOH}$ & $9.23 \times 10^{-12}$ & Atkinson et al. (2004) \\
\hline $\mathrm{C}_{2} \mathrm{H}_{5} \mathrm{OO}+\mathrm{CH}_{3} \mathrm{COOO} \rightarrow \mathrm{CH}_{3} \mathrm{CHO}+\mathrm{CH}_{3} \mathrm{COO}+\mathrm{HO}_{2}$ & $4.0 \times 10^{-12}$ & Michalowski et al. (2000) \\
\hline$i \mathrm{C}_{3} \mathrm{H}_{7} \mathrm{O}_{2}+\mathrm{HO}_{2} \rightarrow i$ Perox & $9.23 \times 10^{-12}$ & Michalowski et al. (2000) \\
\hline$n \mathrm{C}_{3} \mathrm{H}_{7} \mathrm{O}_{2}+\mathrm{HO}_{2} \rightarrow n$ Perox & $9.23 \times 10^{-12}$ & Michalowski et al. (2000) \\
\hline $\mathrm{HCHO}+\mathrm{OH} \rightarrow \mathrm{HO}_{2}+\mathrm{CO}$ & $9.3 \times 10^{-12}$ & Atkinson et al. (2004) \\
\hline $\mathrm{HCHO}+\mathrm{HO}_{2} \rightarrow \mathrm{HOCH}_{2} \mathrm{O}_{2}$ & $7.53 \times 10^{-14}$ & Sander et al. (2006) \\
\hline $\mathrm{HCHO}+\mathrm{NO}_{3} \rightarrow \mathrm{HNO}_{3}+\mathrm{HO}_{2}+\mathrm{CO}$ & $5.8 \times 10^{-16}$ & DeMore et al. (1997) \\
\hline $\mathrm{CH}_{3} \mathrm{CHO}+\mathrm{OH} \rightarrow \mathrm{CH}_{3} \mathrm{COOO}+\mathrm{H}_{2} \mathrm{O}$ & $1.98 \times 10^{-11}$ & Atkinson et al. (2004) \\
\hline $\mathrm{CH}_{3} \mathrm{CHO}+\mathrm{NO}_{3} \rightarrow \mathrm{HNO}_{3}+\mathrm{CH}_{3} \mathrm{COOO}$ & $1.4 \times 10^{-15}$ & DeMore et al. (1997) \\
\hline $\mathrm{CH}_{3} \mathrm{COCH}_{3}+\mathrm{OH} \rightarrow \mathrm{H}_{2} \mathrm{O}+\mathrm{CH}_{3} \mathrm{COCH}_{2}$ & $1.37 \times 10^{-13}$ & Atkinson et al. (2004) \\
\hline $\mathrm{HOCH}_{2} \mathrm{O}_{2}+\mathrm{NO} \rightarrow \mathrm{HCOOH}+\mathrm{HO}_{2}+\mathrm{NO}_{2}$ & $8.68 \times 10^{-12}$ & Lurmann et al. (1986) \\
\hline $\mathrm{HOCH}_{2} \mathrm{O}_{2}+\mathrm{HO}_{2} \rightarrow \mathrm{HCOOH}+\mathrm{H}_{2} \mathrm{O}$ & $2.0 \times 10^{-12}$ & Lurmann et al. (1986) \\
\hline $\mathrm{HOCH}_{2} \mathrm{O}_{2}+\mathrm{HOCH}_{2} \mathrm{O}_{2} \rightarrow \mathrm{HCOOH}+\mathrm{HCOOH}+\mathrm{HO}_{2}+\mathrm{HO}_{2}$ & $1.0 \times 10^{-13}$ & Lurmann et al. (1986) \\
\hline $\mathrm{HCOOH}+\mathrm{OH} \rightarrow \mathrm{HO}_{2}+\mathrm{H}_{2} \mathrm{O}+\mathrm{CO}_{2}$ & $4.0 \times 10^{-13}$ & DeMore et al. (1997) \\
\hline $\mathrm{CH}_{3} \mathrm{COOO}+\mathrm{NO} \rightarrow \mathrm{CH}_{3} \mathrm{OO}+\mathrm{NO}_{2}+\mathrm{CO}_{2}$ & $2.4 \times 10^{-11}$ & Atkinson et al. (2004) \\
\hline $\mathrm{CH}_{3} \mathrm{COOO}+\mathrm{HO}_{2} \rightarrow \mathrm{CH}_{3} \mathrm{COOH}+\mathrm{O}_{3}$ & $1.87 \times 10^{-11}$ & Kirchner and Stockwell (1996) \\
\hline $\mathrm{CH}_{3} \mathrm{COOO}+\mathrm{CH}_{3} \mathrm{COOO} \rightarrow \mathrm{CH}_{3} \mathrm{COO}+\mathrm{CH}_{3} \mathrm{COO}$ & $2.5 \times 10^{-11}$ & Kirchner and Stockwell (1996) \\
\hline $\mathrm{Cl}_{2}+\mathrm{OH} \rightarrow \mathrm{HOCl}+\mathrm{Cl}$ & $2.85 \times 10^{-14}$ & Atkinson et al. (2004) \\
\hline $\mathrm{Cl}+\mathrm{O}_{3} \rightarrow \mathrm{ClO}$ & $1.02 \times 10^{-11}$ & Atkinson et al. (2004) \\
\hline $\mathrm{Cl}+\mathrm{H}_{2} \rightarrow \mathrm{HCl}$ & $3.5 \times 10^{-15}$ & Atkinson et al. (2004) \\
\hline $\mathrm{Cl}+\mathrm{HO}_{2} \rightarrow \mathrm{HCl}$ & $3.57 \times 10^{-11}$ & Sander et al. (2006) \\
\hline $\mathrm{Cl}+\mathrm{HO}_{2} \rightarrow \mathrm{ClO}+\mathrm{OH}$ & $6.68 \times 10^{-12}$ & Sander et al. (2006) \\
\hline $\mathrm{Cl}+\mathrm{H}_{2} \mathrm{O}_{2} \rightarrow \mathrm{HCl}+\mathrm{HO}_{2}$ & $2.11 \times 10^{-13}$ & Atkinson et al. (2004) \\
\hline $\mathrm{Cl}+\mathrm{NO}_{3} \rightarrow \mathrm{ClO}+\mathrm{NO}_{2}$ & $2.4 \times 10^{-11}$ & Atkinson et al. (2004) \\
\hline $\mathrm{Cl}+\mathrm{CH}_{4} \rightarrow \mathrm{HCl}+\mathrm{CH}_{3} \mathrm{OO}$ & $3.99 \times 10^{-14}$ & Sander et al. (2006) \\
\hline $\mathrm{Cl}+\mathrm{C}_{2} \mathrm{H}_{6} \rightarrow \mathrm{HCl}+\mathrm{C}_{2} \mathrm{H}_{5} \mathrm{OO}$ & $5.36 \times 10^{-11}$ & Sander et al. (2006) \\
\hline $\mathrm{Cl}+\mathrm{C}_{2} \mathrm{H}_{4} \rightarrow \mathrm{HCl}+\mathrm{C}_{2} \mathrm{H}_{5} \mathrm{OO}$ & $1.0 \times 10^{-10}$ & Atkinson et al. (2004) \\
\hline $\mathrm{Cl}+\mathrm{MEK} \rightarrow \mathrm{HCl}$ & $4.21 \times 10^{-11}$ & Atkinson et al. (2004) \\
\hline $\mathrm{Cl}+\mathrm{C}_{2} \mathrm{H}_{2} \rightarrow \mathrm{ClC}_{2} \mathrm{CHO}$ & $2.5 \times 10^{-10}$ & Atkinson et al. (2004) \\
\hline $\mathrm{Cl}+\mathrm{C}_{3} \mathrm{H}_{6} \rightarrow \mathrm{HCl}+\mathrm{C}_{3} \mathrm{H}_{6} \mathrm{Cl}$ & $2.7 \times 10^{-10}$ & Keil and Shepson (2006) \\
\hline $\mathrm{Cl}+\mathrm{C}_{3} \mathrm{H}_{8} \rightarrow \mathrm{HCl}+i \mathrm{C}_{3} \mathrm{H}_{7} \mathrm{O}_{2}$ & $1.65 \times 10^{-10}$ & DeMore et al. (1997) \\
\hline $\mathrm{Cl}+\mathrm{C}_{3} \mathrm{H}_{8} \rightarrow \mathrm{HCl}+n \mathrm{C}_{3} \mathrm{H}_{7} \mathrm{O}_{2}$ & $1.65 \times 10^{-10}$ & DeMore et al. (1997) \\
\hline $\mathrm{Cl}+\mathrm{C}_{3} \mathrm{H}_{6} \mathrm{O} \rightarrow \mathrm{HCl}$ & $1.1 \times 10^{-10}$ & Wallington et al. (1988) \\
\hline $\mathrm{Cl}+i \mathrm{C}_{4} \mathrm{H}_{10} \rightarrow \mathrm{HCl}+\mathrm{C}_{4} \mathrm{H}_{9}$ & $1.3 \times 10^{-10}$ & Hooshiyar and Niki (1995) \\
\hline $\mathrm{Cl}+n \mathrm{C}_{4} \mathrm{H}_{10} \rightarrow \mathrm{HCl}+\mathrm{C}_{4} \mathrm{H}_{9}$ & $2.15 \times 10^{-10}$ & Tyndall et al. (1997) \\
\hline $\mathrm{Cl}+\mathrm{n}$-Butanal $\rightarrow \mathrm{HCl}+$ products & $1.1 \times 10^{-10}$ & Michalowski et al. (2000) \\
\hline $\mathrm{Cl}+\mathrm{HCHO} \rightarrow \mathrm{HCl}+\mathrm{HO}_{2}+\mathrm{CO}$ & $7.18 \times 10^{-11}$ & Sander et al. (2006) \\
\hline $\mathrm{Cl}+\mathrm{CH}_{3} \mathrm{CHO} \rightarrow \mathrm{HCl}+\mathrm{CH}_{3} \mathrm{COOO}$ & $8.08 \times 10^{-11}$ & Atkinson et al. (2004) \\
\hline
\end{tabular}


Table 1. Continued.

\begin{tabular}{|c|c|c|}
\hline Reaction & Rate constant & Reference \\
\hline $\mathrm{Cl}+\mathrm{CH}_{3} \mathrm{COCH}_{3} \rightarrow \mathrm{HCl}+\mathrm{CH}_{3} \mathrm{COCH}_{2}$ & $1.39 \times 10^{-12}$ & Atkinson et al. (2004) \\
\hline $\mathrm{Cl}+\mathrm{CH}_{3} \mathrm{OOH} \rightarrow \mathrm{CH}_{3} \mathrm{OO}+\mathrm{HCl}$ & $2.36 \times 10^{-11}$ & Atkinson et al. (2004) \\
\hline $\mathrm{Cl}+\mathrm{CH}_{3} \mathrm{OOH} \rightarrow \mathrm{CH}_{2} \mathrm{OOH}+\mathrm{HCl}$ & $3.54 \times 10^{-11}$ & Atkinson et al. (2004) \\
\hline $\mathrm{Cl}+\mathrm{CHBr}_{3} \rightarrow \mathrm{HCl}+\mathrm{Br}+\mathrm{CBr}_{2} \mathrm{O}$ & $2.9 \times 10^{-13}($ at $298 \mathrm{~K})$ & Kamboures et al. (2002) \\
\hline $\mathrm{Cl}+\mathrm{OClO} \rightarrow \mathrm{ClO}+\mathrm{ClO}$ & $6.35 \times 10^{-11}$ & Atkinson et al. (2004) \\
\hline $\mathrm{Cl}+\mathrm{ClNO}_{3} \rightarrow \mathrm{Cl}_{2}+\mathrm{NO}_{3}$ & $1.12 \times 10^{-11}$ & Sander et al. (2006) \\
\hline $\mathrm{Cl}+\mathrm{PAN} \rightarrow \mathrm{HCl}+\mathrm{HCHO}+\mathrm{NO}_{3}$ & $1.0 \times 10^{-14}$ & Tsalkani et al. (1988) \\
\hline $\mathrm{Cl}+\mathrm{HNO}_{3} \rightarrow \mathrm{HCl}+\mathrm{NO}_{3}$ & $1.0 \times 10^{-16}$ & Wine et al. (1988) \\
\hline $\mathrm{Cl}+\mathrm{NO}_{2} \rightarrow \mathrm{ClNO}_{2}$ & $1.43 \times 10^{-12}($ at $298 \mathrm{~K})$ & Ravishankara et al. (1988) \\
\hline $\mathrm{Cl}+\mathrm{HBr} \rightarrow \mathrm{HCl}+\mathrm{Br}$ & $4.48 \times 10^{-12}$ & Nicovich and Wine (1990) \\
\hline $\mathrm{ClO}+\mathrm{O}\left({ }^{3} P\right) \rightarrow \mathrm{Cl}+\mathrm{O}_{2}$ & $1.6 \times 10^{-11}$ & Atkinson et al. (2004) \\
\hline $\mathrm{ClO}+\mathrm{OH} \rightarrow \mathrm{Cl}+\mathrm{HO}_{2}$ & $2.45 \times 10^{-11}$ & Atkinson et al. (2004) \\
\hline $\mathrm{ClO}+\mathrm{OH} \rightarrow \mathrm{HCl}$ & $2.37 \times 10^{-13}$ & Sander et al. (2006) \\
\hline $\mathrm{ClO}+\mathrm{HO}_{2} \rightarrow \mathrm{HOCl}$ & $8.67 \times 10^{-12}$ & Atkinson et al. (2004) \\
\hline $\mathrm{ClO}+\mathrm{CH}_{3} \mathrm{OO} \rightarrow \mathrm{Cl}+\mathrm{HCHO}+\mathrm{HO}_{2}$ & $2.08 \times 10^{-12}$ & Sander et al. (2006) \\
\hline $\mathrm{ClO}+\mathrm{CH}_{3} \mathrm{COOO} \rightarrow \mathrm{Cl}+\mathrm{CH}_{3} \mathrm{OO}+\mathrm{CO}_{2}$ & $2.03 \times 10^{-12}$ & Michalowski et al. (2000) \\
\hline $\mathrm{ClO}+\mathrm{NO} \rightarrow \mathrm{Cl}+\mathrm{NO}_{2}$ & $2.04 \times 10^{-11}$ & Atkinson et al. (2004) \\
\hline $\mathrm{ClO}+\mathrm{NO}_{2} \rightarrow \mathrm{ClNO}_{3}$ & $7.1 \times 10^{-12}$ & Atkinson et al. (2004) \\
\hline $\mathrm{ClO}+\mathrm{ClO} \rightarrow \mathrm{Cl}_{2}$ & $1.64 \times 10^{-15}$ & Atkinson et al. (2004) \\
\hline $\mathrm{ClO}+\mathrm{ClO} \rightarrow \mathrm{Cl}+\mathrm{Cl}$ & $1.54 \times 10^{-15}$ & Atkinson et al. (2004) \\
\hline $\mathrm{ClO}+\mathrm{ClO} \rightarrow \mathrm{Cl}+\mathrm{OClO}$ & $1.40 \times 10^{-15}$ & Atkinson et al. (2004) \\
\hline $\mathrm{OClO}+\mathrm{OH} \rightarrow \mathrm{HOCl}$ & $1.13 \times 10^{-11}$ & Atkinson et al. (2004) \\
\hline $\mathrm{OClO}+\mathrm{NO} \rightarrow \mathrm{ClO}+\mathrm{H}_{2} \mathrm{O}$ & $1.51 \times 10^{-13}$ & Atkinson et al. (2004) \\
\hline $\mathrm{HOCl}+\mathrm{OH} \rightarrow \mathrm{ClO}+\mathrm{H}_{2} \mathrm{O}$ & $4.0 \times 10^{-13}$ & Sander et al. (2006) \\
\hline $\mathrm{HCl}+\mathrm{OH} \rightarrow \mathrm{Cl}+\mathrm{H}_{2} \mathrm{O}$ & $6.84 \times 10^{-13}$ & Atkinson et al. (2004) \\
\hline $\mathrm{ClNO}_{3}+\mathrm{OH} \rightarrow \mathrm{HOCl}+\mathrm{NO}_{3}$ & $3.17 \times 10^{-13}$ & Atkinson et al. (2004) \\
\hline $\mathrm{HOCl}+\mathrm{O}\left({ }^{3} P\right) \rightarrow \mathrm{ClO}+\mathrm{OH}$ & $1.7 \times 10^{-13}$ & Atkinson et al. (2004) \\
\hline $\mathrm{Br}+\mathrm{O}_{3} \rightarrow \mathrm{BrO}$ & $6.75 \times 10^{-13}$ & Atkinson et al. (2004) \\
\hline $\mathrm{Br}_{2}+\mathrm{OH} \rightarrow \mathrm{HOBr}$ & $5.0 \times 10^{-11}$ & Atkinson et al. (2004) \\
\hline $\mathrm{Br}+\mathrm{HO}_{2} \rightarrow \mathrm{HBr}$ & $1.25 \times 10^{-12}$ & Atkinson et al. (2004) \\
\hline $\mathrm{Br}+\mathrm{C}_{2} \mathrm{H}_{2} \rightarrow \mathrm{BrCH}_{2} \mathrm{CHO}$ & $3.7 \times 10^{-14}$ & Atkinson et al. (2004) \\
\hline $\mathrm{Br}+\mathrm{C}_{2} \mathrm{H}_{4} \rightarrow \mathrm{HBr}+\mathrm{C}_{2} \mathrm{H}_{5} \mathrm{OO}$ & $1.3 \times 10^{-13}$ & Atkinson et al. (2004) \\
\hline $\mathrm{Br}+\mathrm{C}_{3} \mathrm{H}_{6} \rightarrow \mathrm{HBr}+\mathrm{C}_{3} \mathrm{H}_{5}$ & $1.60 \times 10^{-12}$ & Atkinson et al. (2004) \\
\hline $\mathrm{Br}+\mathrm{HCHO} \rightarrow \mathrm{HBr}+\mathrm{CO}+\mathrm{HO}_{2}$ & $6.75 \times 10^{-13}$ & Sander et al. (2006) \\
\hline $\mathrm{Br}+\mathrm{CH}_{3} \mathrm{CHO} \rightarrow \mathrm{HBr}+\mathrm{CH}_{3} \mathrm{COOO}$ & $2.8 \times 10^{-12}$ & Atkinson et al. (2004) \\
\hline $\mathrm{Br}+\mathrm{C}_{3} \mathrm{H}_{6} \mathrm{O} \rightarrow \mathrm{HBr}$ & $9.7 \times 10^{-12}$ & Wallington et al. (1989) \\
\hline $\mathrm{Br}+\mathrm{n}-\mathrm{Butanal} \rightarrow \mathrm{HBr}$ & $9.7 \times 10^{-12}$ & Michalowski et al. (2000) \\
\hline $\mathrm{Br}+\mathrm{CH}_{3} \mathrm{OOH} \rightarrow \mathrm{HBr}+\mathrm{CH}_{3} \mathrm{OO}$ & $4.03 \times 10^{-15}$ & Mallard et al. (1993) \\
\hline $\mathrm{Br}+\mathrm{NO}_{2} \rightarrow \mathrm{BrNO}_{2}$ & $9.41 \times 10^{-13}$ & Atkinson et al. (2004) \\
\hline $\mathrm{Br}+\mathrm{NO}_{2} \rightarrow \mathrm{BrONO}$ & $5.33 \times 10^{-12}$ & Atkinson et al. (2004) \\
\hline $\mathrm{Br}+\mathrm{BrNO}_{3} \rightarrow \mathrm{Br}_{2}+\mathrm{NO}_{3}$ & $4.9 \times 10^{-11}$ & Orlando and Tyndall (1997) \\
\hline $\mathrm{Br}+\mathrm{BrNO}_{2} \rightarrow \mathrm{Br}_{2}+\mathrm{NO}_{2}$ & $5 \times 10^{-11}$ & Orlando and Burkholder (2000) \\
\hline $\mathrm{Br}+\mathrm{BrONO} \rightarrow \mathrm{Br}_{2}+\mathrm{NO}_{2}$ & $1 \times 10^{-12}$ & Orlando et al. (2000) \\
\hline $\mathrm{BrONO} \rightarrow \mathrm{Br}+\mathrm{NO}_{2}$ & 0.02 & Orlando et al. (2000) \\
\hline $\mathrm{BrONO} \rightarrow \mathrm{BrNO}_{2}$ & 0.014 & Orlando et al. (2000) \\
\hline $\mathrm{Br}+\mathrm{OClO} \rightarrow \mathrm{BrO}+\mathrm{ClO}$ & $1.43 \times 10^{-13}$ & Atkinson et al. (2004) \\
\hline $\mathrm{BrO}+\mathrm{O}\left({ }^{3} P\right) \rightarrow \mathrm{Br}$ & $4.8 \times 10^{-11}$ & Atkinson et al. (2004) \\
\hline $\mathrm{BrO}+\mathrm{OH} \rightarrow \mathrm{Br}+\mathrm{HO}_{2}$ & $4.93 \times 10^{-11}$ & Atkinson et al. (2004) \\
\hline $\mathrm{BrO}+\mathrm{HO}_{2} \rightarrow \mathrm{HOBr}$ & $3.38 \times 10^{-11}$ & Atkinson et al. (2004) \\
\hline $\mathrm{BrO}+\mathrm{CH}_{3} \mathrm{OO} \rightarrow \mathrm{HOBr}+\mathrm{CH}_{2} \mathrm{OO}$ & $4.1 \times 10^{-12}$ & Aranda et al. (1997) \\
\hline $\mathrm{BrO}+\mathrm{CH}_{3} \mathrm{OO} \rightarrow \mathrm{Br}+\mathrm{HCHO}+\mathrm{HO}_{2}$ & $1.6 \times 10^{-12}$ & Aranda et al. (1997) \\
\hline $\mathrm{BrO}+\mathrm{CH}_{3} \mathrm{COOO} \rightarrow \mathrm{Br}+\mathrm{CH}_{3} \mathrm{COO}$ & $1.7 \times 10^{-12}$ & Michalowski et al. (2000) \\
\hline $\mathrm{BrO}+\mathrm{C}_{3} \mathrm{H}_{6} \mathrm{O} \rightarrow \mathrm{HOBr}$ & $1.5 \times 10^{-14}$ & Michalowski et al. (2000) \\
\hline
\end{tabular}


Table 1. Continued.

\begin{tabular}{|c|c|c|}
\hline Reaction & Rate constant & Reference \\
\hline $\mathrm{BrO}+\mathrm{NO} \rightarrow \mathrm{Br}+\mathrm{NO}_{2}$ & $2.48 \times 10^{-11}$ & Atkinson et al. (2004) \\
\hline $\mathrm{BrO}+\mathrm{NO}_{2} \rightarrow \mathrm{BrNO}_{3}$ & $1.53 \times 10^{-11}$ & Atkinson et al. (2004) \\
\hline $\mathrm{BrO}+\mathrm{BrO} \rightarrow \mathrm{Br}+\mathrm{Br}$ & $2.82 \times 10^{-12}$ & Sander et al. (2006) \\
\hline $\mathrm{BrO}+\mathrm{BrO} \rightarrow \mathrm{Br}_{2}$ & $9.3 \times 10^{-13}$ & Sander et al. (2006) \\
\hline $\mathrm{BrO}+\mathrm{HBr} \rightarrow \mathrm{HOBr}+\mathrm{Br}$ & $2.1 \times 10^{-14}$ & Hansen et al. (1999) \\
\hline $\mathrm{HBr}+\mathrm{OH} \rightarrow \mathrm{Br}+\mathrm{H}_{2} \mathrm{O}$ & $1.26 \times 10^{-11}$ & Sander et al. (2006) \\
\hline $\mathrm{CH}_{3} \mathrm{Br}+\mathrm{OH} \rightarrow \mathrm{H}_{2} \mathrm{O}+\mathrm{Br}$ & $1.27 \times 10^{-14}$ & Atkinson et al. (2004) \\
\hline $\mathrm{CHBr}_{3}+\mathrm{OH} \rightarrow \mathrm{H}_{2} \mathrm{O}+\mathrm{Br}$ & $1.2 \times 10^{-13}$ & Atkinson et al. (2004) \\
\hline $\mathrm{Cl}+\mathrm{BrCl} \leftarrow \rightarrow \mathrm{Br}+\mathrm{Cl}_{2}$ & $\mathrm{f}: 1.5 \times 10^{-11}$ r: $1.1 \times 10^{-15}$ & Clyne and Cruse (1972) \\
\hline $\mathrm{Cl}+\mathrm{Br}_{2} \longleftrightarrow \mathrm{BrCl}+\mathrm{Br}$ & $\mathrm{f:}: 1.2 \times 10^{-10} \mathrm{r}: 3.3 \times 10^{-1}$ & Clyne and Cruse (1972) \\
\hline $\mathrm{BrO}+\mathrm{ClO} \rightarrow \mathrm{Br}+\mathrm{Cl}$ & $7.04 \times 10^{-12}$ & Atkinson et al. (2004) \\
\hline $\mathrm{BrO}+\mathrm{ClO} \rightarrow \mathrm{BrCl}$ & $1.15 \times 10^{-12}$ & Atkinson et al. (2004) \\
\hline $\mathrm{BrO}+\mathrm{ClO} \rightarrow \mathrm{Br}+\mathrm{OClO}$ & $9.06 \times 10^{-12}$ & Atkinson et al. (2004) \\
\hline $\mathrm{HOBr}+\mathrm{OH} \rightarrow \mathrm{BrO}+\mathrm{H}_{2} \mathrm{O}$ & $5.0 \times 10^{-13}$ & Kukui et al. (1996) \\
\hline $\mathrm{HOBr}+\mathrm{Cl} \rightarrow \mathrm{BrCl}+\mathrm{OH}$ & $8.0 \times 10^{-11}$ & Kukui et al. (1996) \\
\hline $\mathrm{HOBr}+\mathrm{O}\left({ }^{3} P\right) \rightarrow \mathrm{BrO}+\mathrm{OH}$ & $2.12 \times 10^{-11}$ & Atkinson et al. (2004) \\
\hline $\mathrm{I}_{2}+\mathrm{O}\left({ }^{3} P\right) \rightarrow \mathrm{IO}+\mathrm{I}$ & $1.25 \times 10^{-10}$ (at $\left.298 \mathrm{~K}\right)$ & Atkinson et al. (2004) \\
\hline $\mathrm{IO}+\mathrm{O}\left({ }^{3} P\right) \rightarrow \mathrm{I}$ & $1.4 \times 10^{-10}($ at $298 \mathrm{~K})$ & Atkinson et al. (2004) \\
\hline $\mathrm{I}+\mathrm{HO}_{2} \rightarrow \mathrm{HI}$ & $1.85 \times 10^{-13}$ & Atkinson et al. (2004) \\
\hline $\mathrm{I}+\mathrm{O}_{3} \rightarrow \mathrm{IO}$ & $7.39 \times 10^{-13}$ & Atkinson et al. (2004) \\
\hline $\mathrm{I}+\mathrm{NO} \rightarrow \mathrm{INO}$ & $3.48 \times 10^{-13}($ at $298 \mathrm{~K})$ & Atkinson et al. (2004) \\
\hline $\mathrm{I}+\mathrm{NO}_{2} \rightarrow \mathrm{INO}_{2}$ & $5.76 \times 10^{-12}($ at $298 \mathrm{~K})$ & Atkinson et al. (2004) \\
\hline $\mathrm{I}+\mathrm{NO}_{3} \rightarrow \mathrm{IO}+\mathrm{NO}_{3}$ & $1.0 \times 10^{-10}($ at $298 \mathrm{~K})$ & Atkinson et al. (2004) \\
\hline $\mathrm{I}_{2}+\mathrm{NO}_{3} \rightarrow \mathrm{I}+\mathrm{IONO}_{2}$ & $1.5 \times 10^{-12}($ at $298 \mathrm{~K})$ & Atkinson et al. (2004) \\
\hline $\mathrm{HI}+\mathrm{OH} \rightarrow \mathrm{I}+\mathrm{H}_{2} \mathrm{O}$ & $9.43 \times 10^{-11}$ & Atkinson et al. (2004) \\
\hline $\mathrm{I}_{2}+\mathrm{OH} \rightarrow \mathrm{HOI}+\mathrm{I}$ & $2.1 \times 10^{-10}$ & Atkinson et al. (2004) \\
\hline $\mathrm{IO}+\mathrm{NO}_{3} \rightarrow \mathrm{OIO}+\mathrm{NO}_{2}$ & $9.0 \times 10^{-12}($ at $298 \mathrm{~K})$ & Atkinson et al. (2004) \\
\hline $\mathrm{IO}+\mathrm{HO}_{2} \rightarrow \mathrm{HOI}$ & $8.4 \times 10^{-11}$ (at $\left.298 \mathrm{~K}\right)$ & Atkinson et al. (2004) \\
\hline $\mathrm{IO}+\mathrm{ClO} \rightarrow \mathrm{ICl}$ & $3.16 \times 10^{-12}$ & Turnipseed et al. (1997) \\
\hline $\mathrm{IO}+\mathrm{ClO} \rightarrow \mathrm{I}+\mathrm{Cl}$ & $3.95 \times 10^{-12}$ & Turnipseed et al. (1997) \\
\hline $\mathrm{IO}+\mathrm{ClO} \rightarrow \mathrm{I}+\mathrm{OClO}$ & $8.69 \times 10^{-12}$ & Turnipseed et al. 1997) \\
\hline $\mathrm{IO}+\mathrm{BrO} \rightarrow \mathrm{Br}+\mathrm{OIO}$ & $9.36 \times 10^{-11}$ & Rowley et al. (2001) \\
\hline $\mathrm{IO}+\mathrm{BrO} \rightarrow \mathrm{IBr}$ & $4.32 \times 10^{-11}$ & Rowley et al. (2001) \\
\hline $\mathrm{IO}+\mathrm{BrO} \rightarrow \mathrm{Br}+\mathrm{I}$ & $7.2 \times 10^{-12}$ & Rowley et al. (2001) \\
\hline $\mathrm{IO}+\mathrm{IO} \rightarrow \mathrm{I}+\mathrm{OIO}$ & $4.41 \times 10^{-11}$ & Atkinson et al. (2004) \\
\hline $\mathrm{IO}+\mathrm{IO} \rightarrow \mathrm{I}+\mathrm{I}$ & $1.84 \times 10^{-11}$ & Atkinson et al. (2004) \\
\hline $\mathrm{IO}+\mathrm{IO} \leftarrow \rightarrow \mathrm{IOOI}$ & $\mathrm{f}: 5.34 \times 10^{-11} \mathrm{r}: 1.3 \times 10^{-4}$ & Atkinson et al. (2004) \\
\hline $\mathrm{IOOI} \rightarrow \mathrm{OIO}+\mathrm{I}$ & 0.21 & Saiz-Lopez et al. (2008) \\
\hline $\mathrm{IO}+\mathrm{NO} \rightarrow \mathrm{I}+\mathrm{NO}_{2}$ & $1.96 \times 10^{-11}$ & Atkinson et al. (2004) \\
\hline $\mathrm{IO}+\mathrm{NO}_{2} \leftarrow \rightarrow \mathrm{IONO}_{2}$ & f: $4.61 \times 10^{-11}$ r: $8.36 \times 10^{-7}$ & Atkinson et al. (2004) \\
\hline $\mathrm{OIO}+\mathrm{NO} \rightarrow \mathrm{IO}+\mathrm{NO}_{2}$ & $9.78 \times 10^{-12}$ & Atkinson et al. (2004) \\
\hline $\mathrm{OIO}+\mathrm{OH} \rightarrow \mathrm{HOI}$ & $6.0 \times 10^{-12}$ & McFiggans et al. (2002) \\
\hline $\mathrm{HOI}+\mathrm{OH} \rightarrow \mathrm{IO}$ & $2.0 \times 10^{-13}$ & McFiggans et al. (2002) \\
\hline $\mathrm{IO}+\mathrm{OIO} \rightarrow \mathrm{I}_{2} \mathrm{O}_{3}$ & $1.5 \times 10^{-10}$ & Gomez Martin et al. (2005) \\
\hline $\mathrm{OIO}+\mathrm{OIO} \leftarrow \mathrm{I}_{2} \mathrm{O}_{4}$ & $\mathrm{f}: 1.0 \times 10^{-10} \mathrm{r}: 4.4 \times 10^{-4}$ & Sander et al. (2006) \\
\hline $\mathrm{IOOI}+\mathrm{O}_{3} \rightarrow \mathrm{I}_{2} \mathrm{O}_{3}$ & $1.0 \times 10^{-12}$ & Saunders and Plane (2006) \\
\hline $\mathrm{I}_{2} \mathrm{O}_{3}+\mathrm{O}_{3} \rightarrow \mathrm{I}_{2} \mathrm{O}_{4}$ & $1.0 \times 10^{-12}$ & Saunders and Plane (2006) \\
\hline $\mathrm{I}_{2} \mathrm{O}_{4}+\mathrm{O}_{3} \rightarrow \mathrm{I}_{2} \mathrm{O}_{5}$ & $1.0 \times 10^{-12}$ & Saunders and Plane (2006) \\
\hline
\end{tabular}


Table 2. Photochemical reactions. $J_{\max }$ values for 25 March are shown as an example. $J$ coefficients are expressed in units of s ${ }^{-1}$.

\begin{tabular}{|c|c|c|c|}
\hline Reaction & $J_{\max } 25 \mathrm{March}$ & Lifetime & Source \\
\hline $\mathrm{O}_{3} \rightarrow \mathrm{O}_{2}+\mathrm{O}\left({ }^{1} D\right)$ & $3.9 \times 10^{-6}$ & 3.0 days & Calculated from OASIS data \\
\hline $\mathrm{NO}_{2} \rightarrow \mathrm{NO}+\mathrm{O}\left({ }^{3} P\right)$ & $8.6 \times 10^{-3}$ & $1.9 \mathrm{~min}$ & Calculated from OASIS data \\
\hline $\mathrm{H}_{2} \mathrm{O}_{2} \rightarrow \mathrm{OH}+\mathrm{OH}$ & $3.4 \times 10^{-6}$ & 3.4 days & Calculated from OASIS data \\
\hline $\mathrm{NO}_{3} \rightarrow \mathrm{NO}+\mathrm{O}_{2}$ & $4.5 \times 10^{-2}$ & $22 \mathrm{~s}$ & Michalowski et al. (2000) \\
\hline $\mathrm{N}_{2} \mathrm{O}_{5} \rightarrow \mathrm{NO}_{2}+\mathrm{NO}_{3}$ & $1.5 \times 10^{-5}$ & $18 \mathrm{~h}$ & Calculated from OASIS data \\
\hline $\mathrm{HONO} \rightarrow \mathrm{OH}+\mathrm{NO}$ & $1.8 \times 10^{-3}$ & $9.2 \mathrm{~min}$ & Calculated from OASIS data \\
\hline $\mathrm{HNO}_{3} \rightarrow \mathrm{NO}_{2}+\mathrm{OH}$ & $1.5 \times 10^{-7}$ & 79 days & Calculated from OASIS data \\
\hline $\mathrm{HNO}_{4} \rightarrow \mathrm{NO}_{2}+\mathrm{HO}_{2}$ & $7.3 \times 10^{-7}$ & 16 days & Calculated from OASIS data \\
\hline $\mathrm{HCHO} \rightarrow \mathrm{HO}_{2}+\mathrm{HO}_{2}+\mathrm{CO}$ & $1.5 \times 10^{-5}$ & $19 \mathrm{~h}$ & Calculated from OASIS data \\
\hline $\mathrm{HCHO} \rightarrow \mathrm{CO}+\mathrm{H}_{2}$ & $3.1 \times 10^{-5}$ & $8.8 \mathrm{~h}$ & Calculated from OASIS data \\
\hline $\mathrm{CH}_{3} \mathrm{CHO} \rightarrow \mathrm{CH}_{3} \mathrm{OO}+\mathrm{HO}_{2}+\mathrm{CO}$ & $1.1 \times 10^{-6}$ & 11 days & Calculated from OASIS data \\
\hline $\mathrm{CH}_{3} \mathrm{OOH} \rightarrow \mathrm{HCHO}+\mathrm{HO}_{2}+\mathrm{OH}$ & $3.2 \times 10^{-6}$ & 3.7 days & Calculated from OASIS data \\
\hline $\mathrm{C} 3 \mathrm{H} 6 \mathrm{O} \rightarrow \mathrm{HO} 2+\mathrm{C} 2 \mathrm{H} 5 \mathrm{OO}+\mathrm{CO}$ & $1.4 \times 10^{-6}$ & 8.3 days & Calculated from OASIS data \\
\hline $\mathrm{PAN} \rightarrow \mathrm{CH}_{3} \mathrm{COOO}+\mathrm{NO}_{2}$ & $1.7 \times 10^{-7}$ & 66 days & Calculated from OASIS data \\
\hline $\mathrm{OClO} \rightarrow \mathrm{O}\left({ }^{3} P\right)+\mathrm{ClO}$ & 0.12 & $8.1 \mathrm{~s}$ & Estimate from Pöhler et al. (2010) \\
\hline $\mathrm{Cl}_{2} \rightarrow \mathrm{Cl}+\mathrm{Cl}$ & $2.1 \times 10^{-3}$ & $8.1 \mathrm{~min}$ & Calculated from OASIS data \\
\hline $\mathrm{ClO} \rightarrow \mathrm{Cl}+\mathrm{O}\left({ }^{3} P\right)$ & $2.4 \times 10^{-5}$ & $11 \mathrm{~h}$ & Calculated from OASIS data \\
\hline $\mathrm{HOCl} \rightarrow \mathrm{OH}+\mathrm{Cl}$ & $1.4 \times 10^{-4}$ & $2 \mathrm{~h}$ & Estimate from Lehrer et al. (2004) \\
\hline $\mathrm{ClNO}_{3} \rightarrow \mathrm{Cl}+\mathrm{NO}_{3}$ & $2.9 \times 10^{-5}$ & $9.5 \mathrm{~h}$ & Calculated from OASIS data \\
\hline $\mathrm{ClNO}_{3} \rightarrow \mathrm{ClO}+\mathrm{NO}_{2}$ & $3.4 \times 10^{-6}$ & 3.4 days & Calculated from OASIS data \\
\hline $\mathrm{BrNO}_{3} \rightarrow \mathrm{Br}+\mathrm{NO}_{3}$ & $2.1 \times 10^{-4}$ & $1.3 \mathrm{~h}$ & Calculated from OASIS data \\
\hline $\mathrm{BrNO}_{3} \rightarrow \mathrm{BrO}+\mathrm{NO}_{2}$ & $1.2 \times 10^{-3}$ & $14.2 \mathrm{~min}$ & Calculated from OASIS data \\
\hline $\mathrm{BrO} \rightarrow \mathrm{Br}+\mathrm{O}\left({ }^{3} P\right)$ & $3.0 \times 10^{-2}$ & $33 \mathrm{~s}$ & Calculated from OASIS data \\
\hline $\mathrm{Br}_{2} \rightarrow \mathrm{Br}+\mathrm{Br}$ & $4.4 \times 10^{-2}$ & $23 \mathrm{~s}$ & Calculated from OASIS data \\
\hline $\mathrm{HOBr} \rightarrow \mathrm{Br}+\mathrm{OH}$ & $2.3 \times 10^{-3}$ & $7.2 \mathrm{~min}$ & Calculated from OASIS data \\
\hline $\mathrm{BrNO}_{2} \rightarrow \mathrm{Br}+\mathrm{NO}_{2}$ & $1.5 \times 10^{-4}$ & $1.8 \mathrm{~h}$ & Estimate from Lehrer et al. (2004) \\
\hline $\mathrm{ClNO}_{2} \rightarrow \mathrm{Cl}+\mathrm{NO}_{2}$ & $4.4 \times 10^{-5}$ & $6.3 \mathrm{~h}$ & Estimate from Ganske et al. (1992) \\
\hline $\mathrm{BrCl} \rightarrow \mathrm{Br}+\mathrm{Cl}$ & $1.26 \times 10^{-2}$ & $1.3 \mathrm{~min}$ & Calculated from OASIS data \\
\hline $\mathrm{I}_{2} \rightarrow \mathrm{I}+\mathrm{I}$ & 0.15 & $6.7 \mathrm{~s}$ & Calculated from Calvert and Lindberg (2004b) \\
\hline $\mathrm{ICl} \rightarrow \mathrm{I}+\mathrm{Cl}$ & $2.21 \times 10^{-2}$ & $45 \mathrm{~s}$ & Calculated from Calvert and Lindberg (2004b) \\
\hline $\mathrm{IBr} \rightarrow \mathrm{I}+\mathrm{Br}$ & $6.83 \times 10^{-2}$ & $14.6 \mathrm{~s}$ & Calculated from Calvert and Lindberg (2004b) \\
\hline $\mathrm{INO}_{2} \rightarrow \mathrm{I}+\mathrm{NO}_{2}$ & $2.23 \times 10^{-3}$ & $7.5 \mathrm{~min}$ & Calculated from Calvert and Lindberg (2004b) \\
\hline $\mathrm{INO} \rightarrow \mathrm{I}+\mathrm{NO}$ & $8.34 \times 10^{-2}$ & $12 \mathrm{~s}$ & Calculated from Calvert and Lindberg (2004b) \\
\hline $\mathrm{IONO}_{2} \rightarrow \mathrm{IO}+\mathrm{NO}_{2}$ & $7.13 \times 10^{-4}$ & $23.4 \mathrm{~min}$ & Calculated from Calvert and Lindberg (2004b) \\
\hline $\mathrm{IONO}_{2} \rightarrow \mathrm{I}+\mathrm{NO}_{3}$ & $2.91 \times 10^{-4}$ & $57.3 \mathrm{~min}$ & Calculated from Calvert and Lindberg (2004b) \\
\hline $\mathrm{IOOI} \rightarrow \mathrm{I}+\mathrm{I}$ & $1.50 \times 10^{-2}$ & $66.7 \mathrm{~s}$ & Calculated from Calvert and Lindberg (2004b) \\
\hline $\mathrm{IOOI} \rightarrow \mathrm{IO}+\mathrm{IO}$ & $1.50 \times 10^{-2}$ & $66.7 \mathrm{~s}$ & Calculated from Calvert and Lindberg (2004b) \\
\hline $\mathrm{HOI} \rightarrow \mathrm{I}+\mathrm{OH}$ & $5.09 \times 10^{-3}$ & $3.3 \mathrm{~min}$ & Calculated from Calvert and Lindberg (2004b) \\
\hline $\mathrm{IO} \rightarrow \mathrm{I}+\mathrm{O}\left({ }^{3} P\right)$ & 0.18 & $5.6 \mathrm{~s}$ & Calculated from Calvert and Lindberg (2004b) \\
\hline $\mathrm{OIO} \rightarrow \mathrm{IO}+\mathrm{O}\left({ }^{3} P\right)$ & $1.52 \times 10^{-3}$ & $11 \mathrm{~min}$ & Calculated from Calvert and Lindberg (2004b) \\
\hline $\mathrm{OIO} \rightarrow \mathrm{I}$ & $3.26 \times 10^{-2}$ & $30.7 \mathrm{~s}$ & Calculated from Calvert and Lindberg (2004b) \\
\hline
\end{tabular}

position velocities to snow in the Arctic range from 0 to $0.2 \mathrm{~cm} \mathrm{~s}^{-1}$ (Gong et al., 1997; Helmig et al., 2007). Here, we use an average value of $0.05 \mathrm{~cm} \mathrm{~s}^{-1}$ for ozone, similar to the modeling studies of Cavender et al. (2008) and Michalowski et al. (2000). However, there is large uncertainty in this parameter and often-contradictory observations from field measurements (Helmig et al., 2007, 2012). For 25 March, the boundary layer height in Barrow was estimated at $300 \mathrm{~m}$ based on radiosonde data and model simulations (R. Stae- bler, Environment Canada, personal communication, 2010). This corresponds to a $k_{\mathrm{t}}$ for ozone of $1.67 \times 10^{-6} \mathrm{~s}^{-1}$. Because dry deposition velocities to snow have not been determined for the halogen acids, we use the estimation method of Michalowski et al. (2000) and assume a transfer coefficient 10 times greater than that for ozone. Thus, for $\mathrm{HBr}, \mathrm{HCl}$, $\mathrm{HOBr}, \mathrm{HOCl}$, and HOI, $k_{\mathrm{t}}=1.67 \times 10^{-5} \mathrm{~s}^{-1}$ (Table 3). Similarly, we assume an equivalent deposition velocity for the oxidized acidic nitrogen compounds (i.e., $\mathrm{HNO}_{3}, \mathrm{HO}_{2} \mathrm{NO}_{2}$, 
Table 3. Mass transfer reactions. All rate constants are expressed in units of $\mathrm{s}^{-1}$.

\begin{tabular}{|c|c|c|}
\hline Reaction & $k$ (forward) & $k$ (reverse) \\
\hline \multicolumn{3}{|l|}{ Particles } \\
\hline $\begin{array}{l}\mathrm{HCl}_{(\mathrm{g})} \rightarrow \mathrm{H}_{(\mathrm{p})}^{+}+\mathrm{Cl}_{(\mathrm{p})}^{-} \\
\mathrm{HBr}_{(\mathrm{g})} \rightarrow \mathrm{H}_{(\mathrm{p})}^{+}+\mathrm{Br}_{(\mathrm{p})}^{-} \\
\mathrm{HOCl}_{(\mathrm{g})} \rightarrow \mathrm{HOCl}_{(\mathrm{p})} \\
\mathrm{HOBr}_{(\mathrm{g})} \rightarrow \mathrm{HOBr}_{(\mathrm{p})} \\
\mathrm{HOI}_{(\mathrm{g})} \rightarrow \mathrm{HOI}_{(\mathrm{p})} \\
\mathrm{OH}_{(\mathrm{g})} \rightarrow \mathrm{OH}_{(\mathrm{p})} \\
\mathrm{O}_{3(\mathrm{~g})} \leftarrow \rightarrow \mathrm{O}_{3(\mathrm{p})} \\
\mathrm{Cl}_{2(\mathrm{~g})} \leftarrow \rightarrow \mathrm{Cl}_{2(\mathrm{p})} \\
\mathrm{Br}_{2(\mathrm{~g})} \leftarrow \rightarrow \mathrm{Br}_{2(\mathrm{p})} \\
\mathrm{BrCl}_{(\mathrm{g})} \leftarrow \mathrm{BrCl}_{(\mathrm{p})} \\
\mathrm{ICl}_{(\mathrm{p})} \rightarrow \mathrm{ICl}_{(\mathrm{g})} \\
\mathrm{IBr}_{(\mathrm{p})} \rightarrow \mathrm{IBr}_{(\mathrm{g})} \\
\mathrm{HNO}_{3(\mathrm{~g})} \rightarrow \mathrm{HNO}_{3(\mathrm{p})} \\
\mathrm{N}_{2} \mathrm{O}_{5(\mathrm{~g})} \rightarrow \mathrm{N}_{2} \mathrm{O}_{5(\mathrm{p})} \\
\mathrm{HONO}_{(\mathrm{g})} \rightarrow \mathrm{HONO}_{(\mathrm{p})} \\
\mathrm{PAN}_{(\mathrm{g})} \rightarrow \mathrm{PAN}_{(\mathrm{p})} \\
\mathrm{HNO}_{4(\mathrm{~g})} \rightarrow \mathrm{HNO}_{4(\mathrm{p})} \\
\mathrm{ClNO}_{2(\mathrm{p})} \rightarrow \mathrm{ClNO}_{2(\mathrm{~g})} \\
\mathrm{BrNO}_{2(\mathrm{p})} \rightarrow \mathrm{BrNO}_{2(\mathrm{~g})}\end{array}$ & $\begin{array}{r}2.58 \times 10^{-3} \\
1.80 \times 10^{-3} \\
2.16 \times 10^{-3} \\
1.26 \times 10^{-3} \\
5.42 \times 10^{-4} \\
3.26 \times 10^{-5} \\
6.54 \times 10^{-6} \\
2.69 \times 10^{-5} \\
1.78 \times 10^{-5} \\
6.60 \times 10^{-4} \\
2.83 \times 10^{10} \\
5.53 \times 10^{9} \\
5.50 \times 10^{-4} \\
1.08 \times 10^{-4} \\
1.63 \times 10^{-4} \\
2.05 \times 10^{-5} \\
4.89 \times 10^{-4} \\
9.44 \times 10^{3} \\
4.94 \times 10^{4}\end{array}$ & $\begin{array}{r}8.76 \times 10^{5} \\
2.96 \times 10^{7} \\
2.97 \times 10^{8} \\
1.91 \times 10^{10}\end{array}$ \\
\hline \multicolumn{3}{|l|}{ Snow } \\
\hline $\begin{array}{l}\mathrm{HBr}_{(\mathrm{g})} \rightarrow \mathrm{H}_{(\mathrm{s})}^{+}+\mathrm{Br}_{(\mathrm{s})}^{-} \\
\mathrm{HCl}_{(\mathrm{g})} \rightarrow \mathrm{H}_{(\mathrm{s})}^{+}+\mathrm{Cl}_{(\mathrm{s})}^{-} \\
\mathrm{HOBr}_{(\mathrm{g})} \rightarrow \mathrm{HOBr}_{(\mathrm{s})} \\
\mathrm{HOCl}_{(\mathrm{g})} \rightarrow \mathrm{HOCl}_{(\mathrm{s})} \\
\mathrm{HOI}_{(\mathrm{g})} \rightarrow \mathrm{HOI}_{(\mathrm{s})} \\
\mathrm{OH}_{(\mathrm{g})} \rightarrow \mathrm{OH}_{(\mathrm{s})} \\
\mathrm{O}_{3(\mathrm{~g})} \rightarrow \mathrm{O}_{3(\mathrm{~s})} \\
\mathrm{Cl}_{2(\mathrm{~g})} \leftarrow \rightarrow \mathrm{Cl}_{2(\mathrm{~s})} \\
\mathrm{Br}_{2(\mathrm{~g})} \leftarrow \rightarrow \mathrm{Br}_{2(\mathrm{~s})} \\
\mathrm{BrCl}_{(\mathrm{g})} \leftarrow \mathrm{BrCl}_{(\mathrm{s})} \\
\mathrm{ICl}_{(\mathrm{s})} \rightarrow \mathrm{ICl}_{(\mathrm{g})} \\
\mathrm{IBr}_{(\mathrm{s})} \rightarrow \mathrm{IBr}_{(\mathrm{g})} \\
\mathrm{HNO}_{3(\mathrm{~g})} \rightarrow \mathrm{HNO}_{3(\mathrm{~s})} \\
\mathrm{N}_{2} \mathrm{O}_{5(\mathrm{~g})} \rightarrow \mathrm{N}_{2} \mathrm{O}_{5(\mathrm{~s})} \\
\mathrm{HONO}_{(\mathrm{g})} \rightarrow \mathrm{HONO}_{(\mathrm{s})} \\
\mathrm{PAN}_{(\mathrm{g})} \rightarrow \mathrm{PAN}_{(\mathrm{s})} \\
\mathrm{HNO}_{4(\mathrm{~g})} \rightarrow \mathrm{HNO}_{4(\mathrm{~s})} \\
\mathrm{ClNO}_{2(\mathrm{~s})} \rightarrow \mathrm{ClNO}_{2(\mathrm{~g})} \\
\mathrm{BrNO}_{2(\mathrm{~s})} \rightarrow \mathrm{BrNO}_{2(\mathrm{~g})}\end{array}$ & $\begin{array}{r}1.67 \times 10^{-5} \\
1.67 \times 10^{-5} \\
1.67 \times 10^{-5} \\
1.67 \times 10^{-5} \\
1.67 \times 10^{-5} \\
1.67 \times 10^{-6} \\
1.67 \times 10^{-6} \\
8.0 \times 10^{-6} \\
1.0 \times 10^{-5} \\
1.25 \times 10^{-5} \\
7.71 \times 10^{-2} \\
7.71 \times 10^{-2} \\
1.67 \times 10^{-5} \\
1.67 \times 10^{-5} \\
1.67 \times 10^{-5} \\
1.67 \times 10^{-5} \\
1.67 \times 10^{-5} \\
7.71 \times 10^{-2} \\
7.71 \times 10^{-2}\end{array}$ & $\begin{array}{l}7.71 \times 10^{-2} \\
7.71 \times 10^{-2} \\
7.71 \times 10^{-2}\end{array}$ \\
\hline
\end{tabular}

$\mathrm{HONO}, \mathrm{N}_{2} \mathrm{O}_{5}$ ), though a full mechanism of aqueous-phase nitrate chemistry is not included in this model (see Custard et al., 2015, for a more detailed investigation of nitrate chemistry).

As mentioned above, in the final version of our model we used the observed values of $\mathrm{Br}_{2}$ and $\mathrm{Cl}_{2}$ to constrain the halo- gen precursors; however, our model was initially developed to utilize only multiphase chemistry to produce $\mathrm{Br}_{2}\left(\right.$ and $\mathrm{Cl}_{2}$ ) with a mechanism adapted from Michalowski et al. (2000). Transfer coefficients into and out of the particle phase were calculated as described in Jacob (2000), and the snowpack volume available for heterogeneous reaction was limited to a column $10 \mathrm{~cm}$ deep, based on the effective UV extinction depth for Arctic snow (King and Simpson, 2001). Constant concentrations of chloride and bromide ions in the aerosol and snowpack were used throughout the duration of the simulation, as it is likely that these represent an inexhaustible source of chloride and bromide (Lehrer et al., 2004). Concentrations of $\mathrm{Br}^{-}$and $\mathrm{Cl}^{-}$in the snowpack were calculated by assuming all bromide and chloride from bulk snow measurements over "thick first-year ice" near Barrow $(\mathrm{Kr}$ navek et al., 2011) were contained within the quasi-liquid layer (QLL) (Cho et al., 2002). We used a $\mathrm{pH}$ of 4 for the QLL as an estimate, which is in line with previous modeling studies (Thomas et al., 2011, 2012). Aerosol halide concentrations were used as reported for the bulk aerosol chloride and bromide measurements from Barrow, Alaska $(\mathrm{Li}$ and Winchester, 1989), and we used an estimate of $\mathrm{H}^{+}$for Arctic aerosols from the ARCPAC flight campaign (Fisher et al., 2011). Additionally, upward mixing of chemical species emitted from the snowpack heterogeneous reactions was simplified such that it was assumed to be limited only by vertical mixing from the surface and did not include diffusion through the snowpack interstitial air, which is dependent mostly on wind pumping (a parameter not included in this zero-dimensional model). The aqueous-phase reactions included in the model are listed in Table 4.

Using this multiphase mechanism, we were able to produce sufficient gas-phase $\mathrm{Br}_{2}$ in the model solely from the snowpack-aerosol heterogeneous reactions with aqueous bromide; however, we could not reproduce the day-to-day variability in $\left[\mathrm{Br}_{2}\right]$ from only the heterogeneous chemistry; thus, since our main objective was to examine interactions between halogen radical species, we chose to constrain the model to observed $\mathrm{Br}_{2}$. This was necessary to ensure that $[\mathrm{Br}]$ and $[\mathrm{BrO}]$ were accurately represented, so that we could properly examine interactions with other radicals, e.g., $\mathrm{HO}_{2}$, $\mathrm{ClO}$, and $\mathrm{IO}$. There was a period of missing $\mathrm{Br}_{2}$ data during 29 and 30 March. For these days, we used an average $\mathrm{Br}_{2}$ diurnal cycle from the previous days, which was then scaled until the modeled $\mathrm{BrO}$ was in agreement with observations. It should be noted, also, that the uncertainty in the $\mathrm{BrO}$ measurements is high during ODEs as the observed values are very near the detection limit (LOD of $\sim 2$ pptv with an uncertainty of $-3 /+1$ pptv near the LOD), and thus the uncertainty in modeled $[\mathrm{Br}]$ would be greatest during these periods. Only daytime $\mathrm{BrCl}$ was used as produced in the model multiphase mechanism. $\mathrm{BrCl}$ measurements from OASIS are sparse; however, the daytime simulated $\mathrm{BrCl}$ mole ratios of $0-10$ pptv are in agreement with the available observations for the campaign. 
Table 4. Aqueous-phase reactions in the model. All aqueous reaction rate constants are converted to units consistent with the gas-phase reactions to be read by the modeling program.

\begin{tabular}{|c|c|c|c|c|}
\hline Reaction & $k$ (actual) & $k$ (particle) & $k$ (snow) & Reference \\
\hline $\mathrm{Cl}^{-}+\mathrm{HOBr}+\mathrm{H}^{+} \rightarrow \mathrm{BrCl}^{\mathrm{a}}$ & $1.55 \times 10^{-32}$ & $5.17 \times 10^{-21}$ & $9.30 \times 10^{-26}$ & Wang et al. (1994) \\
\hline $\mathrm{Br}^{-}+\mathrm{HOCl}+\mathrm{H}^{+} \rightarrow \mathrm{BrCl}^{\mathrm{a}}$ & $3.59 \times 10^{-36}$ & $1.2 \times 10^{-24}$ & $2.15 \times 10^{-29}$ & Sander et al. (1997) \\
\hline $\mathrm{Br}^{-}+\mathrm{HOBr}+\mathrm{H}^{+} \rightarrow \mathrm{Br}_{2}^{\mathrm{a}}$ & $4.41 \times 10^{-32}$ & $1.47 \times 10^{-20}$ & $2.64 \times 10^{-25}$ & Beckwith et al. (1996) \\
\hline $\mathrm{Cl}^{-}+\mathrm{HOCl}+\mathrm{H}^{+} \rightarrow \mathrm{Cl}_{2}^{\mathrm{a}}$ & $6.07 \times 10^{-38}$ & $2.02 \times 10^{-26}$ & $3.63 \times 10^{-31}$ & Wang and Margerum (1994) \\
\hline $\mathrm{Cl}^{-}+\mathrm{HOI}+\mathrm{H}^{+} \rightarrow \mathrm{ICl}^{\mathrm{a}}$ & $8.01 \times 10^{-32}$ & $2.67 \times 10^{-20}$ & $4.80 \times 10^{-25}$ & Wang et al. (1989) \\
\hline $\mathrm{Br}^{-}+\mathrm{HOI}+\mathrm{H}^{+} \rightarrow \mathrm{IBr}^{\mathrm{a}}$ & $9.12 \times 10^{-30}$ & $3.04 \times 10^{-18}$ & $5.46 \times 10^{-23}$ & Troy et al. (1991) \\
\hline $\mathrm{BrCl}+\mathrm{Cl}^{-} \rightarrow \mathrm{BrCl}_{2}^{-\mathrm{b}}$ & $1 \times 10^{-11}$ & 3.3 & $5.99 \times 10^{-5}$ & Michalowski et al. (2000) \\
\hline $\mathrm{BrCl}_{2}^{-} \rightarrow \mathrm{BrCl}+\mathrm{Cl}_{2}^{-\mathrm{c}}$ & $1.58 \times 10^{9}$ & $1.58 \times 10^{9}$ & $1.58 \times 10^{9}$ & Michalowski et al. (2000) \\
\hline $\mathrm{BrCl}+\mathrm{Br}^{-} \rightarrow \mathrm{Br}_{2} \mathrm{Cl}^{-\mathrm{b}}$ & $1 \times 10^{-11}$ & 3.3 & $5.99 \times 10^{-5}$ & Michalowski et al. (2000) \\
\hline $\mathrm{Br}_{2} \mathrm{Cl}^{-} \rightarrow \mathrm{BrCl}+\mathrm{Br}^{-\mathrm{c}}$ & $3.34 \times 10^{5}$ & $3.34 \times 10^{5}$ & $3.34 \times 10^{5}$ & Wang et al. (1994); Michalowski et al. (2000) \\
\hline $\mathrm{Cl}_{2}+\mathrm{Br}^{-} \rightarrow \mathrm{BrCl}_{2}^{-\mathrm{b}}$ & $1.28 \times 10^{-11}$ & 4.27 & $7.66 \times 10^{-5}$ & Wang et al. (1994); Michalowski et al. (2000) \\
\hline $\mathrm{BrCl}_{2}^{-} \rightarrow \mathrm{Cl}_{2}+\mathrm{Br}^{-} \mathrm{c}$ & $6.94 \times 10^{2}$ & $6.94 \times 10^{2}$ & $6.94 \times 10^{2}$ & Wang et al. (1994); Michalowski et al. (2000) \\
\hline $\mathrm{O}_{3}+\mathrm{Br}^{-} \rightarrow \mathrm{HOBr}^{\mathrm{b}}$ & $1.35 \times 10^{-20}$ & $4.5 \times 10^{-9}$ & $8.08 \times 10^{-14}$ & Oum et al. (1998b); Michalowski et al. (2000) \\
\hline $\mathrm{OH}+\mathrm{Cl}^{-} \rightarrow \mathrm{HOCl}^{\mathrm{b}}$ & $1.35 \times 10^{-20}$ & $4.5 \times 10^{-9}$ & $8.08 \times 10^{-14}$ & Assumed same as $\mathrm{O}_{3}+\mathrm{Br}^{-}$ \\
\hline $\mathrm{N}_{2} \mathrm{O}_{5}+\mathrm{Cl}^{-} \rightarrow \mathrm{ClNO}_{2}^{\mathrm{b}}$ & $1.66 \times 10^{-12}$ & $5.5 \times 10^{-1}$ & $9.94 \times 10^{-5}$ & Assume diffusion limited \\
\hline $\mathrm{ClNO}_{2}+\mathrm{H}^{+}+\mathrm{Cl}^{-} \rightarrow \mathrm{Cl}_{2}^{\mathrm{b}}$ & $1.66 \times 10^{-14}$ & $5.5 \times 10^{-3}$ & $9.94 \times 10^{-8}$ & Estimated from Roberts et al. (2008) \\
\hline $\mathrm{N}_{2} \mathrm{O}_{5}+\mathrm{Br}^{-} \rightarrow \mathrm{BrNO}_{2}^{\mathrm{b}}$ & $1.66 \times 10^{-12}$ & $5.5 \times 10^{-1}$ & $9.94 \times 10^{-5}$ & Assume diffusion limited \\
\hline $\mathrm{BrNO}_{2}+\mathrm{H}^{+}+\mathrm{Br}^{-} \rightarrow \mathrm{Br}_{2}^{\mathrm{b}}$ & $7.31 \times 10^{-17}$ & $2.44 \times 10^{-5}$ & $4.38 \times 10^{-10}$ & Estimated from Schweitzer et al. (1998) \\
\hline
\end{tabular}

${ }^{a}$ Third-order rate constant, expressed in units of $\mathrm{cm}^{6}$ molecule ${ }^{-2} \mathrm{~s}^{-1}$.

b Second-order rate constant, expressed in units of $\mathrm{cm}^{3}$ molecule ${ }^{-1} \mathrm{~s}^{-1}$.

${ }^{\mathrm{c}}$ First-order rate constant, expressed in units of $\mathrm{s}^{-1}$.

Table 5. Summary of the ambient measurements from OASIS that were used to constrain the model and the instrumental method used.

\begin{tabular}{lll}
\hline Measured species & Method & Reference \\
\hline $\mathrm{O}_{3}, \mathrm{NO}, \mathrm{NO}_{2}$ & Chemiluminescence & $\begin{array}{l}\text { Ridley et al. (1992), } \\
\text { Ryerson et al. (2000) }\end{array}$ \\
$\mathrm{CO}$ & IR absorption CO analyzer \\
$\mathrm{Cl}_{2}, \mathrm{Br}_{2}, \mathrm{BrO}, \mathrm{HOBr}$ & Chemical ionization mass \\
& spectrometry (CIMS) & Liao et al. (2011, 2012, 2014) \\
$\mathrm{ClO}$ & Chemical reaction GC-ECD & \\
$\mathrm{HCHO}$ & Tunable diode laser absorption & Stephens et al. (2012) \\
$\mathrm{HONO}$ & spectroscopy & Fried et al. (1997), \\
$\mathrm{CH}_{3} \mathrm{CHO}, \mathrm{CH}_{3} \mathrm{COCH}_{3}, \mathrm{MEK}$, & Long-path absorption photometer & Lancaster et al. (2000) \\
$n-\mathrm{C}_{4} \mathrm{H}_{10}, i-\mathrm{C}_{4} \mathrm{H}_{10}$ & Online fast GC-MS & Villena et al. (2011) \\
$\mathrm{C}_{2} \mathrm{H}_{2}, \mathrm{C}_{2} \mathrm{H}_{4}, \mathrm{C}_{2} \mathrm{H}_{6}, \mathrm{C}_{3} \mathrm{H}_{8}, \mathrm{C}_{3} \mathrm{H}_{6}$, & Canister samples, offline GC-MS & Apel et al. (2010) \\
$n-\mathrm{C}_{4} \mathrm{H}_{10}, i-\mathrm{C}_{4} \mathrm{H}_{10}$ & & Russo et al. (2010) \\
\hline
\end{tabular}

Volumetric fluxes were also introduced into the model for $\mathrm{HONO}$ and $\mathrm{I}_{2}$. The fluxes of $\mathrm{HONO}$ and $\mathrm{I}_{2}$ were scaled to $\mathrm{J}_{\mathrm{NO} 2}$ since $\mathrm{HONO}$, and likely $\mathrm{I}_{2}$, are photochemically produced (Honrath et al., 1999; Zhou et al., 2001; Saiz-Lopez et al., 2011). The HONO flux was adjusted to agree with observed gas-phase concentrations (Villena et al., 2011), with daytime averages near 10 pptv and spikes in excess of 20 pptv, as shown in Fig. 3a. Although the flux is included, and is necessary for measured HONO to agree with observations across all 7 days of the simulation, the contribution of the flux is small in comparison to gas-phase production through $\mathrm{NO}_{x}$ chemistry, with the exception of 26-28 March, when gas-phase $\mathrm{NO}_{x}$ is especially low. Figure $3 \mathrm{~b}$ shows a time series of the $\mathrm{NO}$ and $\mathrm{NO}_{2}$ observations that has been re-scaled to a smaller range on the $y$ axis so that the relatively higher $\mathrm{NO}_{x}$ on 29 and 30 March can be discerned. A recent paper by $\mathrm{Li}$ et al. (2014) speculates a possible additional gas-phase source of $\mathrm{HONO}$ from the reaction of $\mathrm{NO}_{2}$ with an $\mathrm{HO}_{2} \times \mathrm{H}_{2} \mathrm{O}$ complex. However, as laboratory studies are still required to quantify the efficiency of this mecha- 
nism, the rate constant of this reaction, and the product yield of HONO, this mechanism was not included in our model.

To investigate the impacts that different levels of iodine chemistry could have on ozone depletions, two iodine scenarios were used, which will be referred to as "Low Iodine" and "High Iodine." For the Low Iodine case, the $\mathrm{I}_{2}$ flux was chosen such that average daytime gas-phase mole ratios of IO remain near 1 pptv (i.e., near or below the detection limit of DOAS and in line with previous indications of [IO] in the Arctic) for the majority of days (as shown in Fig. 4). Only during the ozone depletion period did simulated IO reach close to 2 pptv, for the chosen $\mathrm{I}_{2}$ flux. The higher [IO] during ozone-depleted periods is a result of the constant $\mathrm{I}_{2}$ flux that is utilized for iodine, in contrast to bromine and chlorine species, which use actual observations. Because the $\mathrm{I}_{2}$ flux is constant during the $\mathrm{ODE}$, while $\mathrm{Br}_{2}$ and especially $\mathrm{Cl}_{2}$ are decreased, iodine becomes more dominant for reaction with the remaining $\mathrm{O}_{3}(\sim 1-2$ ppbv) and [IO] increases. $\mathrm{I}_{2}$ averages $\sim 0.025$ pptv throughout the Low Iodine simulation. The High Iodine scenario uses a flux that is adjusted so that $\mathrm{I}_{2}$ averages $0.5 \mathrm{pptv}$ across the simulated period. This results in IO mole ratios ranging from 5 to 10 pptv (see Fig. 3).

\section{Results and discussion}

\subsection{Comparison of modeled and observed mole ratios for select species}

Those species that are not specifically prescribed in the model (e.g., all radical species and many inorganic halogen compounds) by inputting time-varying observations or by introducing a flux are allowed to freely evolve. Here we compare modeled vs. observed mole ratios for only those species that are most important for this analysis in halogen interactions.

Since the contribution of each halogen atom to ozone destruction is a function of its concentration, it is important that the model simulates halogen radicals at levels that are consistent with observations. Comparisons of the multi-day model output with observed $\mathrm{BrO}$ and $\mathrm{ClO}$ are shown in Fig. $2 \mathrm{~b}$ and e. $\mathrm{ClO}$ observations are limited, but the model captures the occurrence and general shape of the $\mathrm{ClO}$ peak observed on 29 March. However, the model generally underpredicts $\mathrm{ClO}$ where there are available observations. A question to address is the extent to which there are other sources of $\mathrm{Cl}$ atoms during this time, such as $\mathrm{HOCl}$ or chlorinated organic compounds that are not included here. In Fig. 2b, the simulated $\mathrm{BrO}$ output generated through bromine chemistry constrained by observed $\mathrm{Br}_{2}$ is compared with $\mathrm{BrO}$ observations. The model represents the overall temporal profile and magnitude of [BrO] throughout this period. This is important for the analysis of the interactions between $\mathrm{BrO}, \mathrm{ClO}$, and $\mathrm{IO}$, which is the focus of this work. It has been suggested that daytime $\mathrm{Br}_{2}$ observed by CIMS contains a contribution from

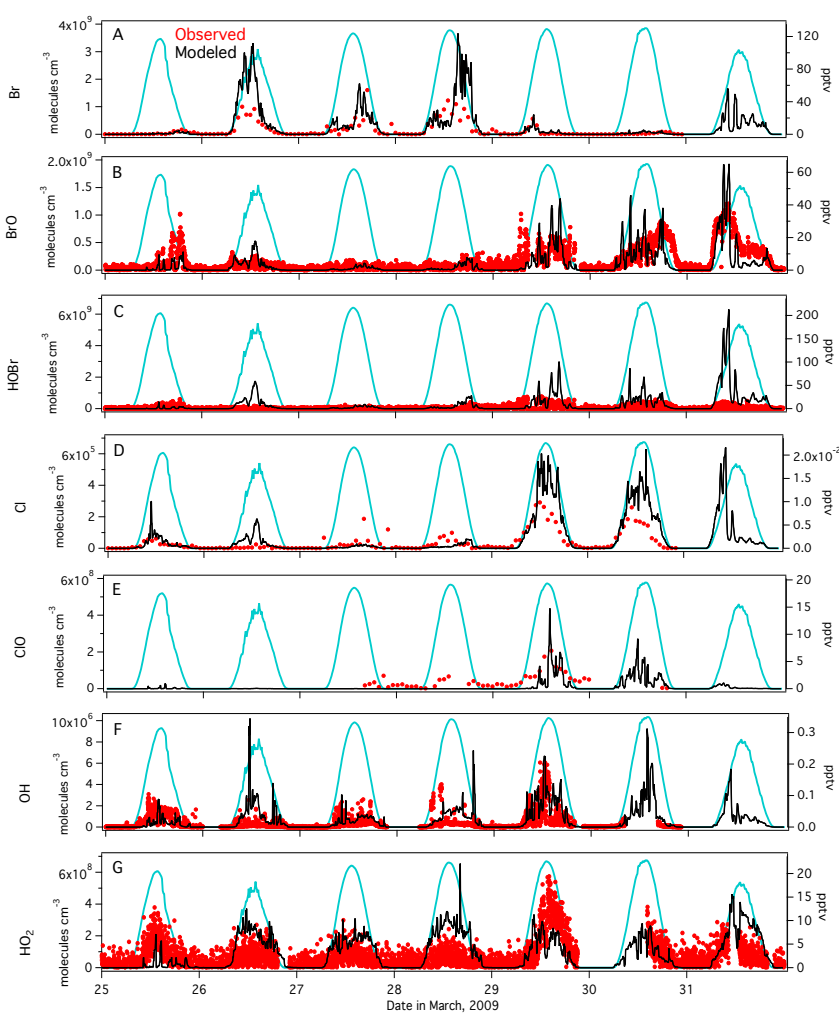

Figure 2. Modeled (black) compared to observed (red) time series for $\mathrm{BrO}, \mathrm{HOBr}, \mathrm{ClO}, \mathrm{OH}$, and $\mathrm{HO}_{2}$. Modeled $\mathrm{Br}$ and $\mathrm{Cl}$ (black) are compared to steady-state approximations as calculated in Stephens et al. (2012) (red). All concentrations are shown in molecules $\mathrm{cm}^{-3}$ on the left axis and as mole ratios in pptv on the right axis. Radiation is shown as the cyan trace as a reference. Time is expressed in Alaska Standard Time.

$\mathrm{HOBr}$ conversion to $\mathrm{Br}_{2}$ on the inlet, and that daytime $\mathrm{Br}_{2}$ should be below detection limits on average due its fast photolysis rate (Liao et al., 2012). However, this modeling study suggests that $\mathrm{Br}_{2}$ should indeed be present in the daytime (given the agreement with observed [BrO]), though it is acknowledged that there is some degree of interference from $\mathrm{HOBr}$, as is apparent from the model overprediction of $\mathrm{BrO}$ on 31 March. Considering an $e$-folding photolytic lifetime of $\mathrm{Br}_{2}$ at solar noon of $23 \mathrm{~s}\left(J_{\max }=0.044 \mathrm{~s}^{-1}\right)$, and using the method of Guimbaud et al. (2002), the effective daytime mixing height $\left(Z^{*}\right)$ of $\mathrm{Br}_{2}$ in the stable air typical of the Arctic $\left(K_{c}=95 \mathrm{~cm}^{2} \mathrm{~s}^{-1}\right)$ is $\sim 0.5 \mathrm{~m}$. Assuming simple first-order kinetics, the $\left[\mathrm{Br}_{2}\right]$ remaining after mixing up from the surface to the intake of the CIMS ( $\sim 1 \mathrm{~m}$ or 2 lifetimes) is $10 \%$ that at the surface. A recent study examining $\mathrm{Br}_{2}$ production from surface snow in Barrow demonstrates that enhanced $\mathrm{Br}_{2}$ production is observed in the presence of solar radiation (Pratt et al., 2013). Given that the $\mathrm{Br}_{2}$ concentrations in the snowpack interstitial air should be elevated due to heterogeneous production mechanisms (e.g., the bromine explosion), and that production should be greater during sunlit periods, it seems 


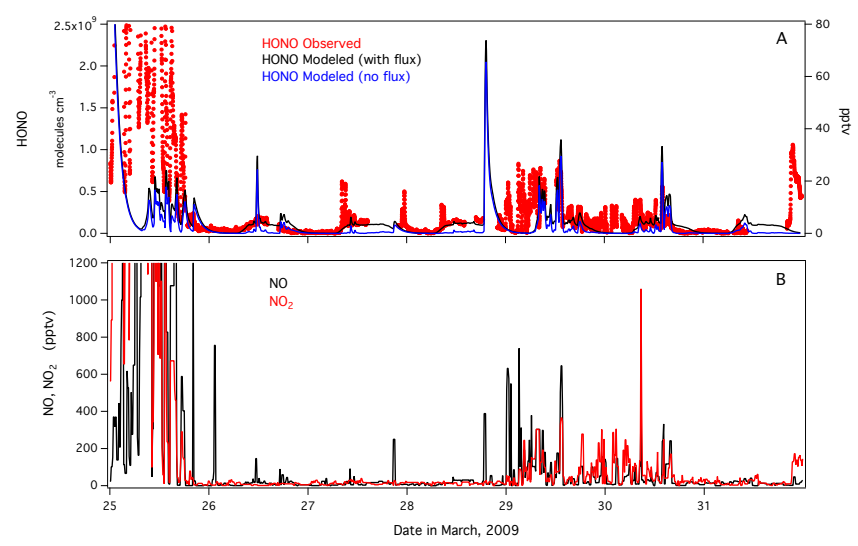

Figure 3. (a) Time series of in situ HONO observations (red data) and modeled HONO including an additional flux (black trace) and without an added flux (blue data). (b) Time series of observed NO (black trace) and $\mathrm{NO}_{2}$ (red trace) that has been re-scaled to a smaller range on the $y$ axis to show the relatively higher $\mathrm{NO}_{x}$ mole ratios on 29 and 30 March.

reasonable to conclude that $\mathrm{Br}_{2}$ should sometimes be observable during the day. Indeed, $\mathrm{Br}_{2}$ mole ratios needed in the model to reproduce $\mathrm{BrO}$ observations agree with the "uncorrected" $\mathrm{Br}_{2}$ observations (shown as the red data in Fig. 2a), as reported in Liao et al. (2012).

Figure $2 \mathrm{a}$ and $\mathrm{d}$ compare the model output for $\mathrm{Br}$ and $\mathrm{Cl}$ atom concentrations (black trace), with steady-state approximations of $[\mathrm{Br}]$ and $[\mathrm{Cl}]$ (red trace) following the method of Stephens et al. (2012), as direct observations of $\mathrm{Br}$ and $\mathrm{Cl}$ atoms have not yet been achieved. In both cases, the modelsimulated output is greater than the steady-state approximations on a few days, sometimes by as much as $90 \%$, although both methods capture the diurnal trends and fluctuations. A notable feature of Fig. $2 \mathrm{a}$ are the rather high $\mathrm{Br}$ atom concentrations during the 3-day ODE, with concentrations up to $3 \times 10^{9}$ molecules $\mathrm{cm}^{-3}(\sim 100 \mathrm{pptv})$, though as mentioned previously, this should be considered an upper limit due to the uncertainty of the $\mathrm{Br}_{2}$ and $\mathrm{BrO}$ measurements and the potentially positive model bias during this time. Nonetheless, higher $[\mathrm{Br}]$ (and $[\mathrm{I}]$ ) is expected during periods of complete ozone depletion due to the loss of this dominant $\mathrm{Br}$ atom chemical sink. However, these concentrations are on the high end of previous estimates using hydrocarbon measurements, which range from $1 \times 10^{6}$ to $1 \times 10^{9}$ for ODEs (Jobson et al., 1994; Ariya et al., 1998; Rudolph et al., 1999). While [Br] peaks during periods of low $\mathrm{O}_{3},[\mathrm{Cl}]$ is enhanced when $\mathrm{O}_{3}$ is present due to the elevated $\mathrm{Cl}_{2}$ mole ratios that are observed only when $\mathrm{O}_{3}$ is above $\sim 10$ ppbv (Fig. 1a; Liao et al., 2014). The model predicts $\mathrm{Cl}$ atom concentrations of $2 \times 10^{5}$ to $6 \times 10^{5}$ molecules $\mathrm{cm}^{-3}$, which is also higher than previous estimates of $1 \times 10^{3}$ to $1 \times 10^{5}$ determined from hydrocarbon measurements (Jobson et al., 1994; Ariya et al., 1998; Rudolph et al., 1999; Boudries and Bottenheim, 2000; Keil and Shepson, 2006). As discussed in Stephens et al. (2012),

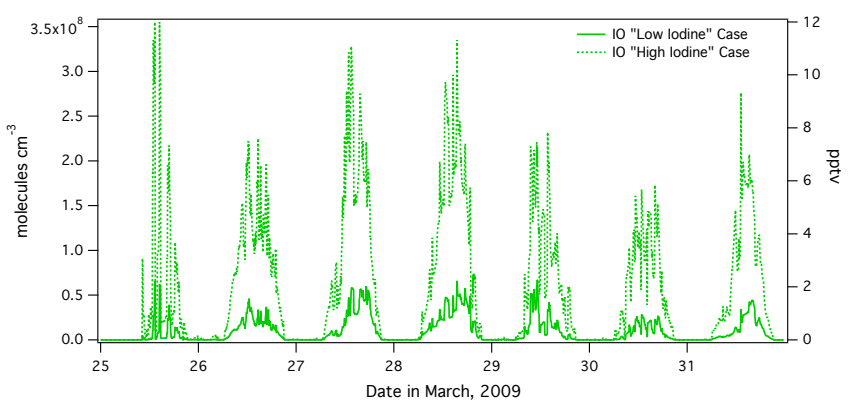

Figure 4. Simulated time series of IO shown for the "Low Iodine" (solid line) and "High Iodine" (dashed line) scenarios. The "Low Iodine" scenario and the "High Iodine" scenario correspond to a daytime average of 0.025 and 0.5 pptv $\mathrm{I}_{2}$, respectively. Time is expressed in Alaska Standard Time.

the hydrocarbon-based methods average over the transport path, which can be aloft and consist of several days, and thus should be lower than that observed near the surface, if the surface is the $\mathrm{Cl}_{2}$ and $\mathrm{Br}_{2}$ source.

Model simulations of $[\mathrm{OH}]$ and $\left[\mathrm{HO}_{2}\right]$ (Fig. $2 \mathrm{f}$ and g) lie within the bounds of measurement uncertainty for the ambient data, with the exception of 29 March, when the model underpredicts $\mathrm{HO}_{2}$ by a factor of $\sim 3$. The accurate simulation of $\mathrm{OH}$ and $\mathrm{HO}_{2}$ is important since halogen chemistry, especially that involving chlorine, can have a significant impact on the $\mathrm{HO}_{x}$ budget. The discrepancy between the modeled and observed $\mathrm{HO}_{2}$ for 29 March may lead to a less than realistic impact of $\mathrm{HO}_{2}$ on oxidation chemistry for this day. The model captures the temporal cycle of $\mathrm{HOBr}$ well; however, it generally overpredicts the daytime peak mole ratios (Fig. 2c). It is unclear why this should be the case, given that both $\mathrm{BrO}$ and $\mathrm{HO}_{2}$ are well represented, with the exception of the $\mathrm{BrO}$ overprediction early on 31 March. This suggests that either our simple parameterization of deposition to snow and particle surfaces is slower than that occurring in nature, or that we are missing some other important HOBr sink.

\subsection{Contribution of $\mathrm{Br}, \mathrm{Cl}$, and I to ozone depletion and the rate and timescale of ozone loss}

An important question regarding ozone depletion events is the contribution of the halogen radicals, $\mathrm{Br}, \mathrm{Cl}$, and $\mathrm{I}$, to the total destruction of ozone and the rate of ozone depletion. Based on field measurements of reactive bromine compounds, primarily $\mathrm{BrO}$, it is generally accepted that bromine is the dominant reactant leading to ozone destruction (Simpson et al., 2007). However, this has not been quantified over highly variable chemical conditions, since prior to OASIS, simultaneous high-time resolution measurements of such a large suite of chemical species had not been performed. Few studies have examined the impact of $\mathrm{Cl}$ on ozone depletion, and these have not had the benefit of in situ observations of $\mathrm{Cl}_{2}$ and $\mathrm{ClO}$ with which to compare their models. This ob- 
servational data set and model thus represent a unique opportunity.

In this study, "Base Model" runs are those that include both bromine and chlorine. "Br Only" simulations are performed by removing $\mathrm{Cl}_{2}$ and chloride; likewise, " $\mathrm{Cl}$ Only" simulations are performed by removing $\mathrm{Br}_{2}$, bromide, and organobromine compounds. Simulations with iodine are conducted by introducing fluxes of $\mathrm{I}_{2}$ of a magnitude necessary to keep the average daytime IO near 1 pptv (Low Iodine) or at 0.5 pptv $\mathrm{I}_{2}$ (High Iodine), as described previously (reference Fig. 4). It is important to note that the model is adjusted to fit observations using the Base Model only. No re-adjustments are made to the model when either bromine or chlorine is turned off, or when iodine is turned on, to observe the effect of a one-variable change.

The time-varying fraction of ozone depleted by bromine, chlorine, and iodine was calculated by considering those reactions that destroy ozone (i.e., Reaction R2 for $\mathrm{Br}$ and the analogous reactions for $\mathrm{Cl}$ and $\mathrm{I}$ ) while correcting for those reactions that result in the regeneration of $\mathrm{O}_{3}$. Here, photolysis of $\mathrm{BrO}, \mathrm{ClO}$, or $\mathrm{IO}$, as well as reaction of $\mathrm{XO}$ with $\mathrm{NO}$, ultimately lead to $\mathrm{O}_{3}$ production. The rate of $\mathrm{O}_{3}$ loss by halogen $X$ is determined by Eq. (2), where $X=\mathrm{Br}, \mathrm{Cl}$, or I, and the total chemical $\mathrm{O}_{3}$ loss rate is calculated using Eq. (3). The fractional contribution for each halogen is then determined by dividing the two quantities.

$$
\text { Rate of } \begin{aligned}
\mathrm{O}_{3} \text { loss by } X & =k[X]\left[\mathrm{O}_{3}\right]-k[X \mathrm{O}][\mathrm{NO}] \\
& -J[X \mathrm{O}]
\end{aligned}
$$

$$
\begin{aligned}
\text { Total } \mathrm{O}_{3} \text { loss rate } & =k[\mathrm{Br}]\left[\mathrm{O}_{3}\right]+k[\mathrm{Cl}]\left[\mathrm{O}_{3}\right]+k[\mathrm{I}]\left[\mathrm{O}_{3}\right] \\
& +k\left[\mathrm{O}\left({ }^{1} D\right)\right]\left[\mathrm{H}_{2} \mathrm{O}\right]+k[\mathrm{OH}]\left[\mathrm{O}_{3}\right] \\
& +k\left[\mathrm{HO}_{2}\right]\left[\mathrm{O}_{3}\right]-k[\mathrm{BrO}][\mathrm{NO}] \\
& -J[\mathrm{BrO}]-k[\mathrm{ClO}][\mathrm{NO}] \\
& -J[\mathrm{ClO}]-k[\mathrm{IO}][\mathrm{NO}]-J[\mathrm{IO}]
\end{aligned}
$$

While we recognize that considering only the sum of the rates of $X \mathrm{O}+X \mathrm{O}$ reactions (where $X=\mathrm{Br}, \mathrm{Cl}$, or $\mathrm{I}$ ) has generally be used in previous studies to estimate the rate of ozone destruction (Le Bras and Platt, 1995; Platt and Janssen, 1995), we have chosen to use Eqs. (2) and (3) as it is likely that these complex interactions are oversimplified by the $X \mathrm{O}+X \mathrm{O}$ method. Indeed, we show in a forthcoming manuscript (Thompson et al., 2015) that the $X \mathrm{O}+X \mathrm{O}$ method underestimates chemical ozone loss by $\mathrm{Br}$ and $\mathrm{Cl}$ atoms when compared to Eq. (2).

Figure 5 shows the fraction of the rate of $\mathrm{O}_{3}$ depletion by $\mathrm{Br}$ and $\mathrm{Cl}$ for the Base, the Base + Low Iodine, and the Base + High Iodine model scenarios as a diurnal average for the daytime hours of only 25 and 29-30 March, for conditions in which $\mathrm{O}_{3}>5 \mathrm{ppbv}$, since this quantity is only meaningful when $\mathrm{O}_{3}$ is present. $\mathrm{Br}$ accounts for the vast majority of $\mathrm{O}_{3}$ depletion in the Base scenario, at about $95 \%$, whereas

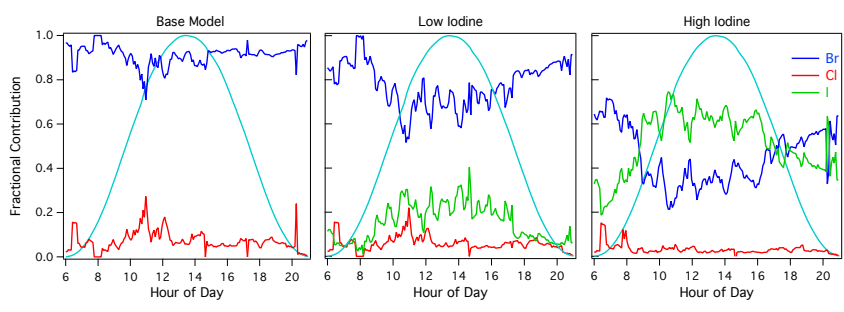

Figure 5. Average diurnal cycles of the fraction of $\mathrm{O}_{3}$ depleted by $\mathrm{Br}, \mathrm{Cl}$ and $\mathrm{I}$ atoms for the Base Model scenario, the Base + Low Iodine scenario and the Base + High Iodine scenario. $\mathrm{Br}$ is shown in the blue trace, $\mathrm{Cl}$ in the red trace, and $\mathrm{I}$ in the green trace; radiation is shown in the cyan trace as a reference. Data shown are only for periods when $\mathrm{O}_{3}>5$ ppbv. Time is expressed in Alaska Standard Time.

$\mathrm{Cl}$ only accounts for $5-10 \%$ of ozone loss on average. In the Low Iodine plot, it can be seen that the levels of iodine considered ( 1 ppt IO) contribute more to $\mathrm{O}_{3}$ destruction than does chlorine on average, with a contribution of $20-40 \%$. This also decreases the Br contribution to $60-80 \%$. When High Iodine is included, the relative importance of $\mathrm{Br}$ and I switches, such that I now contributes $\sim 60 \%$ to ozone loss, whereas $\mathrm{Br}$ is reduced to $30-40 \%$. Thus, it is clear that iodine can have a significant contribution to ozone depletion even at low levels.

To investigate this in further detail, we used a focused version of our model that simulated a $1.5 \mathrm{~h}$ period fixed at mid-day conditions to facilitate instantaneous calculations of ozone depletion. We used chemical mole ratios similar to those of 29 March. This day has $\mathrm{Br}_{2}$ at $6.7 \mathrm{pptv}$ and $\mathrm{Cl}_{2}$ at $80 \mathrm{pptv}$, and ozone at background concentrations near 30 ppbv. We chose not to use 25 March, as this day was impacted by local pollution during the first half of the day (as evidenced by $\mathrm{NO}_{x}$ and $\mathrm{CO}$ in Fig. 1). The mid-day version of our model was run with constant molecular halogen and VOC concentrations; thus, although the conditions are similar to 29 March, the results are not directly comparable.

The fractional contribution of each halogen to ozone destruction was calculated using Eq. (2) for ten different model scenarios with different combinations of halogens present and with no halogens. In Eq. (2), $P, Q, R, S, T, U$, and $V$ represent the total value for model "counters" for each process in parentheses, and the denominator is the sum of chemical ozone loss terms. Because photolysis of $\mathrm{O}_{3}$ can result in fast reformation of $\mathrm{O}_{3}$ through the reaction of $\mathrm{O}\left({ }^{3} \mathrm{P}\right)+\mathrm{O}_{2}$, the $\mathrm{O}\left({ }^{1} \mathrm{D}\right)+\mathrm{H}_{2} \mathrm{O}$ reaction is used to represent the ozone that is destroyed via photolysis.

$$
\begin{aligned}
F_{X}= & \frac{P\left(X+\mathrm{O}_{3}\right)-Q\left(J_{X \mathrm{O}}\right)-R(X \mathrm{O}+\mathrm{NO})}{P\left(X+\mathrm{O}_{3}\right)-Q\left(J_{X \mathrm{O}}\right)-R(X \mathrm{O}+\mathrm{NO})} \\
& +S\left(\mathrm{O}_{3}+\mathrm{OH}\right)+T\left(\mathrm{O}_{3}+\mathrm{HO}_{2}\right) \\
& +U\left(\mathrm{O}_{3}+\mathrm{NO}_{2}\right)+V\left(\mathrm{O}^{1} D+\mathrm{H}_{2} \mathrm{O}\right)
\end{aligned}
$$

Results of this calculation are shown in Table 6 for the scenarios studied. In addition to the fraction of $\mathrm{O}_{3}$ loss by each 
Table 6. Fraction of ozone depleted by each halogen calculated for mid-day of 29 March via Eq. (4). "Br and Cl” is the Base Model.

\begin{tabular}{lrrrrrr}
\hline Model conditions & $\begin{array}{r}\Delta \mathrm{O}_{3} \\
(\mathrm{ppbv})\end{array}$ & $\begin{array}{r}\mathrm{Total} \mathrm{O}_{3} \\
\text { Production } \\
\text { (ppbv) }\end{array}$ & $\begin{array}{r}\mathrm{Sum} \mathrm{O}_{3} \\
\text { Loss terms } \\
(\mathrm{ppbv})\end{array}$ & $\begin{array}{r}\% \text { of } \mathrm{O}_{3} \\
\text { Loss by } \mathrm{Br}\end{array}$ & $\begin{array}{r}\% \text { of } \mathrm{O}_{3} \\
\text { Loss by Cl }\end{array}$ & $\begin{array}{r}\% \text { of } \mathrm{O}_{3} \\
\text { Loss by I }\end{array}$ \\
\hline No Halogens & 0.05 & 0.07 & 0.01 & - & - & - \\
Br Only & -2.57 & 0.46 & 2.24 & 98.6 & - & - \\
Cl Only & 0.39 & 0.89 & 0.36 & - & 81.9 & - \\
Low I Only & -0.12 & 0.07 & 0.16 & - & - & 92.1 \\
High I Only & -2.39 & 0.09 & 1.68 & - & - & 99.1 \\
$\mathrm{Br}$ and Cl (Base) & -4.09 & 0.86 & 4.15 & 87.1 & 11.5 & - \\
$\mathrm{Br}$ and Low I & -3.36 & 0.46 & 2.94 & 86.9 & - & 11.9 \\
$\mathrm{Br}$ and High I & -7.17 & 0.45 & 6.08 & 64.4 & - & 35.0 \\
$\mathrm{Br}, \mathrm{Cl}$, and Low I & -4.91 & 0.85 & 4.95 & 80.2 & 9.72 & 8.94 \\
$\mathrm{Br}, \mathrm{Cl}$, and High I & -8.43 & 0.83 & 7.92 & 64.5 & 5.99 & 28.8 \\
\hline
\end{tabular}

halogen, Table 6 also shows the total change in $\mathrm{O}_{3}$ across the simulated period $\left(\Delta \mathrm{O}_{3}\right)$, the total $\mathrm{O}_{3}$ production via the $\mathrm{O}\left({ }^{3} \mathrm{P}\right)+\mathrm{O}_{2}$ reaction, and the sum of the $\mathrm{O}_{3}$ loss terms (denominator of Eq. 4).

It is clear from Table 6 that $\mathrm{Br}$ is the primary driver of $\mathrm{O}_{3}$ loss, with an at least $64 \%$ contribution in all cases in which it is present, reaching up to $87 \%$ in the " $\mathrm{Br}$ and $\mathrm{Cl}$ " (Base) scenario and $97 \%$ in the "Br Only" simulation. In the "Br and $\mathrm{Cl}$ " run, $\mathrm{Cl}$ accounts for $11.5 \%$ of the loss (at $\mathrm{Cl}_{2}$ levels somewhat enhanced at $80 \mathrm{pptv}$ ), which is a minor, though significant, contribution. On an absolute basis, the addition of chlorine chemistry also enhances the $\mathrm{O}_{3}$ depletion caused by bromine, with the total ozone depleted by $\mathrm{Br}$ increasing from $2.21 \mathrm{ppbv}$ in the Br Only scenario to $3.61 \mathrm{ppbv}$ in the $\mathrm{Br}$ and $\mathrm{Cl}$ scenario (data not shown). This is primarily a result of the efficient cross-reaction between $\mathrm{BrO}$ and $\mathrm{ClO}$ that regenerates $\mathrm{Br}$ and thereby increases the $[\mathrm{Br}] /[\mathrm{BrO}]$ ratio. Comparing "Br Only" with "Br and Low I", it is apparent that iodine chemistry directly contributes a significant amount to ozone depletion, at a fractional contribution of $11.9 \%$ with just 1 ppt IO present, due to both the fast reaction between $\mathrm{I}$ and $\mathrm{O}_{3}$, and $\mathrm{IO}$ and $\mathrm{BrO}$, as well as the lack of known competing sinks for I. When iodine is increased, as in the "Br and High I" scenario, the iodine contribution jumps to $35.0 \%$. From examination of the fractional ozone depletion, it appears that iodine chemistry decreases the $\mathrm{Br}$ destruction of ozone; however, on an absolute basis, there is an increase in the total amount of ozone destroyed by $\mathrm{Br}$ in the " $\mathrm{Br}$ and Low I" simulation (2.55 ppbv $\mathrm{O}_{3}$ destroyed) compared to the "Br Only" simulation (2.21 ppbv $\left.\mathrm{O}_{3}\right)$; this is even greater in the " $\mathrm{Br}$ and High I" scenario, at $3.92 \mathrm{ppbv} \mathrm{O}_{3}$. The $\Delta \mathrm{O}_{3}$ also increases by a factor of 1.3 for the "Br and Low I" case and 2.8 for the "Br and High I" case over the integrated $1.5 \mathrm{~h}$ simulation period. When Low Iodine is added to the Base Model, there is a factor of 1.2 increase in $\Delta \mathrm{O}_{3}$, with iodine contributing $\sim 9 \%$ to the ozone depletion; this increases to a factor of 3.3 increase in $\Delta \mathrm{O}_{3}$ and $28.8 \%$ contribution from I when High Iodine is included. In the " $\mathrm{Br}, \mathrm{Cl}$, and Low I" case, $\mathrm{Cl}$ atoms actually contribute more to the ozone depletion; however, this is a function of the much higher $\mathrm{Cl}_{2}(80 \mathrm{pptv})$ than $\mathrm{I}_{2}(0.025 \mathrm{pptv})$ in this scenario. On a per atom basis, iodine is more efficient than chlorine at depleting ozone. That is partly due to the fact that $\sim 50-100 \%$ of I atoms are lost via reaction with $\mathrm{O}_{3}$, while only $\sim 20 \%$ of $\mathrm{Cl}$ atoms are lost via $\mathrm{O}_{3}$ reaction.

The presence of multiple halogen species leads to synergistic effects, where the total amount of $\mathrm{O}_{3}$ destroyed is greater than the sum of the species run in isolation. This synergism is primarily due to the efficient cross-reactions between $\mathrm{BrO}, \mathrm{ClO}$, and $\mathrm{IO}$ that reform $\mathrm{O}_{3}$-depleting halogen atoms. The absolute ozone destruction by $\mathrm{Br}$ is enhanced more by iodine than by chlorine as a result of the very fast reaction between $\mathrm{BrO}$ and $\mathrm{IO}$. Indeed, the increase of $1.41 \mathrm{ppbv}$ of $\mathrm{O}_{3}$ destroyed by $\mathrm{Br}$ when chlorine is included (" $\mathrm{Br}$ and $\mathrm{Cl}$ " case vs. "Br Only" case) corresponds to an increase of only 0.02 ppbv of $\mathrm{O}_{3}$ destroyed per pptv of $\mathrm{Cl}_{2}$, whereas the enhancement in ozone destruction by $\mathrm{Br}$ provided by low iodine is nearly 7 ppbv of $\mathrm{O}_{3}$ per pptv of $\mathrm{I}_{2}$. Therefore, while $\mathrm{Cl}$ acts primarily to enhance $\mathrm{Br}$ chemistry, I both increases the efficiency of bromine catalyzed ozone destruction and directly depletes ozone.

Chlorine is an especially interesting case because it contributes to both $\mathrm{O}_{3}$ depletion (though relatively minor) and $\mathrm{O}_{3}$ production. Indeed, in the " $\mathrm{Cl}$ Only" run, there is a net increase in $\mathrm{O}_{3}$ that is greater than in the "No Halogens" scenario by a factor of nearly 8 . This increase is due to the efficient reaction of $\mathrm{Cl}$ with nearly all VOCs, and the resultant production of $\mathrm{RO}_{2}$ and $\mathrm{HO}_{2}$. $\mathrm{Br}$, in contrast, does not react appreciably with most VOCs, although its reaction with $\mathrm{HCHO}$ will also lead to $\mathrm{HO}_{2}$ production, as evidenced by the increase in $\mathrm{O}_{3}$ production in the "Br Only" scenario compared to the "No Halogens" case. I does not react to produce $\mathrm{HO}_{2}$; thus, there is no additional $\mathrm{O}_{3}$ production in the "Low I Only" case, and only a slight increase in the "High I Only" case, likely as a result of $\mathrm{IO}$ reaction with $\mathrm{NO}$ to form $\mathrm{NO}_{2}$. It is clear that fully understanding ODE chemistry will re- 
quire a complete understanding of all three halogen radical families.

Platt and Janssen (1995) indicate that $~ 99 \%$ of $\mathrm{Br}$ atoms react with $\mathrm{O}_{3}$ when present at background levels, while only $\sim 50 \%$ of $\mathrm{Cl}$ atoms react with $\mathrm{O}_{3}$ due to efficient reactions with many VOCs. However, this quantity is highly dependent on the mole ratios of $\mathrm{O}_{3}, \mathrm{HCHO}, \mathrm{CH}_{3} \mathrm{CHO}$, and the VOCs, which can fluctuate independently of each other. To further investigate how the three halogens contribute to ozone depletion, we considered the fraction of available $\mathrm{Br}, \mathrm{Cl}$, and I atoms that react with $\mathrm{O}_{3}$ over all other competing pathways across the 7-day simulation with iodine included (Low Iodine) as shown in Fig. 6. The inclusion of iodine does not significantly change the result for $\mathrm{Br}$ and $\mathrm{Cl}$; thus, only the simulation including $\mathrm{I}$ is shown. $\mathrm{Br}$ atoms were simulated to react with $\mathrm{O}_{3} 55-95 \%$ of the time when $\mathrm{O}_{3}$ is not depleted, but this fraction fluctuates significantly and is at times below $25 \%$ when $\mathrm{O}_{3}$ is depleted (i.e., $<5 \mathrm{ppbv}$ ). $\mathrm{Cl}$ atom reaction with $\mathrm{O}_{3}$ varies between 10 and $20 \%$ on average, and is always less than $30 \%$ for this time period. These numbers are lower than those estimated previously, likely because we are using actual measurements of all of the known $\mathrm{Br}$ and $\mathrm{Cl}$ atom sinks that contribute to this quantity, e.g., $\mathrm{HCHO}$, $\mathrm{CH}_{3} \mathrm{CHO}$, numerous VOCs, and $\mathrm{NO}_{x}$. Based on our chemical mechanism, I atoms can react with $\mathrm{O}_{3}$ up to $100 \%$ of the time, and usually up to $90 \%$ (though with high variability during $\mathrm{O}_{3}$-depleted periods), consistent with the estimates of Platt and Janssen (1995), who determined $99 \%$ for I. However, as stated previously, it is likely that our iodine reaction scheme is incomplete, e.g., for IO reaction with peroxy radicals.

Not all halogen atom reactions with $\mathrm{O}_{3}$ result in a net loss of ozone. Though up to $90 \%$ of $\mathrm{Br}$ atoms react with $\mathrm{O}_{3}$, most of these reactions do not ultimately destroy ozone, as it can be reformed via, e.g., $\mathrm{BrO}$ photolysis or $\mathrm{BrO}$ reaction with $\mathrm{NO}$. When considering only $\mathrm{Br}$ and $\mathrm{Cl}$ in our mid-day model, $70 \%$ of the $\mathrm{BrO}$ formed regenerates $\mathrm{O}_{3}$ through photolysis or reaction with $\mathrm{NO}$, whereas this quantity is only $12 \%$ for $\mathrm{ClO}$, which photolyzes much more slowly. While this suggests that $\mathrm{Cl}$ atoms are actually more efficient at destroying ozone on a per atom basis, the lower concentration of $\mathrm{Cl}$ due to the numerous competing $\mathrm{Cl}$-atom sinks and the low fraction of $\mathrm{Cl}$ atoms that react with $\mathrm{O}_{3}$ make it a minor player in ozone depletion. When iodine is included at low levels, thus opening the $\mathrm{BrO}+\mathrm{IO}$ cross-reaction, the percentage of $\mathrm{BrO}$ that reforms $\mathrm{O}_{3}$ drops to $64 \%$ and that for IO is comparable at $65 \%$.

A highly significant finding from the OASIS 2009 campaign was the observation of unexpectedly high levels of $\mathrm{Cl}_{2}$ (Liao et al., 2014). Given the observed $\mathrm{Cl}_{2}$ maxima of 100 $400 \mathrm{pptv}$, a sensitivity study was performed to investigate the impact that such high concentrations could have on ozone depletion chemistry. Table 7 shows the results of modeling runs for the mid-day of 29 March performed by varying $\mathrm{Cl}_{2}$ between 25 and 400 pptv (recall that the Base scenario here

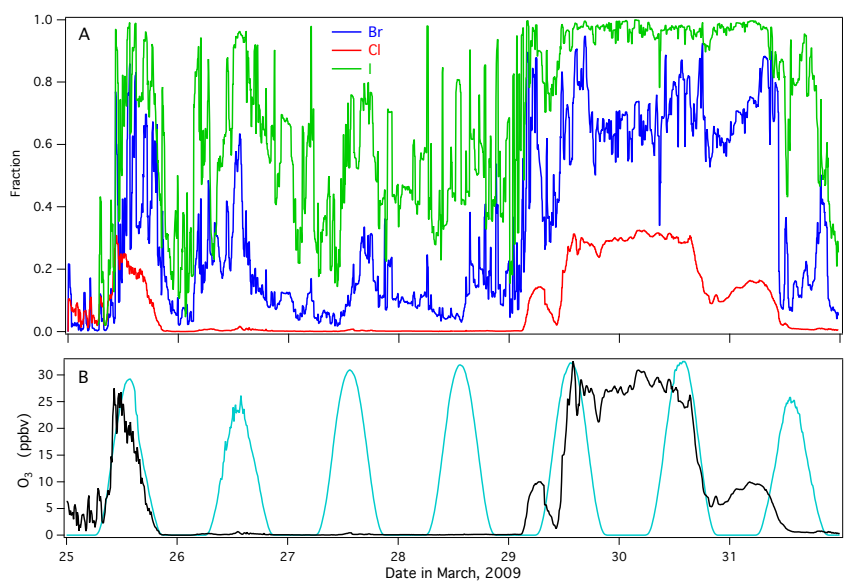

Figure 6. (a) Time-varying fraction of $\mathrm{Br}$ (blue), $\mathrm{Cl}$ (red), and $\mathrm{I}$ (green) atoms that react with $\mathrm{O}_{3}$ across the 7 days of the simulation. (b) Time series of observed ozone mole ratios with radiation is shown as the cyan trace as a reference. Time is expressed in Alaska Standard Time.

for $29 \mathrm{March}$ has $80 \mathrm{pptv} \mathrm{Cl}_{2}$ ). These particular runs included only bromine and chlorine chemistry. Here it can be seen that the high $\mathrm{Cl}_{2}$ levels observed in Barrow can have significant impacts on $\mathrm{O}_{3}$ depletion, with $\mathrm{Cl}$ atoms accounting for over $30 \%$ of $\mathrm{O}_{3}$ loss when present at 400 pptv. At $\mathrm{Cl}_{2}$ levels less than 100 pptv, however, $\mathrm{Cl}$ destruction of $\mathrm{O}_{3}$ is $\leq 11 \%$; thus, chlorine must be relatively elevated to make an appreciable impact on $\mathrm{O}_{3}$ depletion. While the amount of $\mathrm{O}_{3}$ production also increases at higher $\mathrm{Cl}_{2}$ (by an additional $0.58 \mathrm{ppbv} \mathrm{O}_{3}$ at $400 \mathrm{pptv} \mathrm{Cl}_{2}$ compared to $25 \mathrm{pptv} \mathrm{Cl}_{2}$ ), the increase in the $\mathrm{O}_{3}$ destruction by both the additional $\mathrm{Cl}$ atoms $\left(2.8 \mathrm{ppbv} \mathrm{O}_{3}\right)$ and by the enhanced efficiency of $\mathrm{Br}$ atoms through the synergy of the halogen cross-reactions $\left(4.37\right.$ ppbv $\left.\mathrm{O}_{3}\right)$ far outweighs the $\mathrm{O}_{3}$ production. Thus, when both bromine and chlorine are present, our modeling results do not show indications of a "chlorine counter-cycle" hindering ozone depletion as suggested by Piot and von Glasow (2009).

It is useful to also consider the rate of ozone depletion. Often, fast apparent ozone depletions, in which $\mathrm{O}_{3}$ is observed to decrease over timescales of hours, have been attributed to air mass transport of ozone-depleted air, whereas local chemistry is believed to result in a more gradual depletion (Bottenheim and Chan, 2006; Simpson et al., 2007; Halfacre et al., 2014). The ozone depletion rate and the resulting timescale for depletion induced by $\mathrm{Br}, \mathrm{Cl}$, and I, both in isolation (for $\mathrm{Br}$ and $\mathrm{I}$ ) and when allowed to interact, were investigated with model runs conducted for "Br Only" and "I Only", as well as with different combinations of the above, including both Low Iodine and High Iodine conditions. $\mathrm{Cl}$ was not run in isolation here, since as was shown in Table 6, the "Cl Only" simulation did not result in a net loss of $\mathrm{O}_{3}$. Here, we considered only the ozone depletion rate at midday of $29 \mathrm{March}$, determined using the delta $\mathrm{O}_{3}$ for the $1.3 \mathrm{~h}$ 
Table 7. Fraction of ozone depleted by bromine and chlorine calculated for mid-day of 29 March via Eq. (4) for modeling scenarios performed by varying $\mathrm{Cl}_{2}$ mole ratios.

\begin{tabular}{lrrrrr}
\hline Model conditions & $\begin{array}{r}\Delta \mathrm{O}_{3} \\
(\mathrm{ppbv})\end{array}$ & $\begin{array}{r}\mathrm{Total} \mathrm{O}_{3} \\
\text { Production } \\
\text { (ppbv) }\end{array}$ & $\begin{array}{r}\mathrm{Sum} \mathrm{O}_{3} \\
\text { Loss terms } \\
(\mathrm{ppbv})\end{array}$ & $\begin{array}{r}\% \text { of } \mathrm{O}_{3} \\
\text { Loss by } \mathrm{Br}\end{array}$ & $\begin{array}{r}\% \text { of } \mathrm{O}_{3} \\
\text { Loss by } \mathrm{Cl}\end{array}$ \\
\hline 25 pptv $\mathrm{Cl}_{2}$ & -3.15 & 0.62 & 2.87 & 94.1 & 4.30 \\
50 pptv Cl & -3.60 & 0.74 & 3.46 & 90.6 & 7.80 \\
$100 \mathrm{pptv} \mathrm{Cl}_{2}$ & -4.36 & 0.92 & 4.55 & 85.3 & 13.5 \\
200 pptv $\mathrm{Cl}_{2}$ & -5.66 & 1.13 & 6.55 & 78.1 & 21.0 \\
400 pptv $\mathrm{Cl}_{2}$ & -7.96 & 1.20 & 10.05 & 70.4 & 29.1 \\
\hline
\end{tabular}

Table 8. Ozone depletion rates in $\mathrm{ppbvh}^{-1}$ calculated by Eq. (3) and the corresponding timescale for ozone depletion from 35 to 5 ppbv for mid-day of 29 March for eight different modeling scenarios with different combinations of halogen radicals present. " $\mathrm{Br}$ and $\mathrm{Cl}$ " is the Base Model.

\begin{tabular}{lrr}
\hline Model conditions & $\begin{array}{r}\text { Ozone depletion } \\
\text { rate }\left(\mathrm{ppbv} \mathrm{h}^{-1}\right)\end{array}$ & $\begin{array}{r}\text { Depletion timescale } \\
(35 \text { to } 5 \mathrm{ppbv})\end{array}$ \\
\hline Br Only & 1.85 & $16.2 \mathrm{~h}$ \\
Low Iodine Only & 0.09 & 13.9 days \\
High Iodine Only & 1.72 & $17.4 \mathrm{~h}$ \\
Br and Low Iodine & 2.42 & $12.4 \mathrm{~h}$ \\
$\mathrm{Br}$ and High Iodine & 5.16 & $5.81 \mathrm{~h}$ \\
$\mathrm{Br}$ and Cl (Base) & 2.94 & $10.2 \mathrm{~h}$ \\
$\mathrm{Br}, \mathrm{Cl}$, and Low Iodine & 3.54 & $8.47 \mathrm{~h}$ \\
$\mathrm{Br}, \mathrm{Cl}$, and High Iodine & 6.07 & $4.94 \mathrm{~h}$ \\
\hline
\end{tabular}

simulation time (as shown in Table 6), to calculate the depletion timescale. Table 8 shows the resulting ozone depletion rates in $\mathrm{ppbv} \mathrm{h}^{-1}$ for the different permutations of halogen radicals studied, along with the resultant timescale for total ozone depletion from a background of 35 to 5 ppbv, our definition of a major ODE (assuming a constant ozone depletion rate, which represents an upper limit as the rate of ozone depletion decreases as $\left[\mathrm{O}_{3}\right]$ decreases).

For the chemical conditions observed on this day, bromine chemistry alone is capable of depleting ozone on a timescale of $16.2 \mathrm{~h}$ at a rate of $1.85 \mathrm{ppbv} \mathrm{h}^{-1}$, whereas low iodine levels would require 13.9 days at a rate of $0.09 \mathrm{ppbv} \mathrm{h}^{-1}$. High iodine alone depletes ozone at a rate of $1.72 \mathrm{ppbv} \mathrm{h}^{-1}$ in $17.4 \mathrm{~h}$. Again, the synergy that exists between the halogen species is apparent, such that the combination of any halogen species depletes ozone at a faster rate than the sum of the components run in isolation. This is a result that was previously found for the interaction of bromine and iodine in modeling studies by Calvert and Lindberg (2004a, b), Saiz-Lopez et al. (2008) and Mahajan et al. (2010). The greatest ozone depletion rate of $6.07 \mathrm{ppbr}^{-1}$, with a timescale for complete depletion of only $4.9 \mathrm{~h}$, results when all three halogens are present, with $\mathrm{I}_{2}$ set at the High Iodine level of $0.5 \mathrm{pptv}$. Based on these results, it can be concluded that iodine has the potential to have a much greater impact on ozone depletion than chlorine. The combination of $\mathrm{Br}, \mathrm{Cl}$ and low iodine, resulting in an $8.5 \mathrm{~h}$ depletion timescale, is consistent with the fast-end timescales observed over the Beaufort Sea by Halfacre et al. (2014).

It should be noted, however, that the ozone lifetimes discussed above simply represent the calculated ozone lifetime, as determined from measurements conducted within the surface layer. As discussed in detail in Tackett et al. (2007), the current view of the boundary layer is one that is very chemically stratified, with the most important source of $\mathrm{Br}_{2}$ and $\mathrm{Cl}_{2}$ likely from the snowpack surface. Thus, the concept of a "boundary layer" is one that has been defined in terms of the height over which ozone is observed to be depleted, i.e., typically $\sim 400 \mathrm{~m}$ (Bottenheim et al., 2002; Helmig et al., 2012). It is likely, however, that ozone is depleted largely in the very near-surface layer, and the timescale for that depletion is thus determined in significant part by the timescale for downward diffusion of ozone in the $0-400 \mathrm{~m}$ range to the near-surface layer in which $\mathrm{BrO}_{x}$ concentrations are large. In short, when averaged over the entire boundary layer, the actual ozone depletion timescale may potentially be slower than that calculated here using only surface measurements, because of diffusion limitation.

In Table 9, we show the average ozone depletion rate (calculated as discussed for Table 8) for the five different scenarios with varying $\left[\mathrm{Cl}_{2}\right]$. When $\mathrm{Cl}_{2}$ is present at only $25 \mathrm{pptv}$, the timescale for depletion is $13.2 \mathrm{~h}$, which is longer than for the Base Model (with 80 pptv $\mathrm{Cl}_{2}$ ), but still less than 1 day, primarily due to the activity of bromine. $100 \mathrm{pptv}$ $\mathrm{Cl}_{2}$ decreases the timescale for depletion to $9.55 \mathrm{~h}$, while at $400 \mathrm{pptv}$, the timescale is only $5.24 \mathrm{~h}$ (again assuming constant $\mathrm{Cl}_{2}$ mole ratios). For comparison, the Base Model with low iodine as shown in Table 8 has an ozone depletion timescale of $8.47 \mathrm{~h}$, whereas the Base Model with High Iodine has a depletion timescale of $4.94 \mathrm{~h}$. Thus, the presence of only 0.025 pptv of $\mathrm{I}_{2}$ has a greater effect on the ozone depletion rate than does $100 \mathrm{pptv}$ of $\mathrm{Cl}_{2}$, and sustained levels of $\mathrm{Cl}_{2}$ greater than 400 pptv would be required to have a comparable impact on ozone depletion, as does $0.5 \mathrm{pptv} \mathrm{I}_{2}$. 
Table 9. Ozone depletion rates in $\mathrm{ppbv} \mathrm{h}^{-1}$ calculated by Eq. (3) and the corresponding timescale for ozone depletion from 35 to 5 ppbv for mid-day of 29 March for five different modeling scenarios performed by varying $\mathrm{Cl}_{2}$ mole ratios.

\begin{tabular}{lrr}
\hline Model conditions & $\begin{array}{r}\text { Ozone depletion } \\
\text { rate }\left(\mathrm{ppbv} \mathrm{h}^{-1}\right)\end{array}$ & $\begin{array}{r}\text { Depletion timescale } \\
(35 \mathrm{ppbv} \text { to } 5 \mathrm{ppbv})\end{array}$ \\
\hline $25 \mathrm{pptv} \mathrm{Cl}_{2}$ & 2.27 & $13.2 \mathrm{~h}$ \\
$50 \mathrm{pptv} \mathrm{Cl}_{2}$ & 2.59 & $11.6 \mathrm{~h}$ \\
$100 \mathrm{pptv} \mathrm{Cl}_{2}$ & 3.14 & $9.55 \mathrm{~h}$ \\
$200 \mathrm{pptv} \mathrm{Cl}_{2}$ & 4.08 & $7.35 \mathrm{~h}$ \\
$400 \mathrm{pptv} \mathrm{Cl}_{2}$ & 5.73 & $5.24 \mathrm{~h}$ \\
\hline
\end{tabular}

Although the depletion timescales reported here, both for Base Model conditions and for elevated $\left[\mathrm{Cl}_{2}\right]$, represent relatively fast ozone depletion and, as stated, represent an upper limit assuming a constant ozone depletion rate, total ozone depletions are often observed to occur on timescales of a day or less (Tang and McConnell, 1996; Simpson et al., 2007; Halfacre et al., 2014), and a fast ozone depletion of $\sim 7 \mathrm{~h}$ attributed to local chemistry was reported over the Arctic Ocean by Jacobi et al. (2006). Thus, the ozone depletion rates calculated here (including for elevated $\left[\mathrm{Cl}_{2}\right]$ ) show that rapid photochemical ozone depletion events are possible, and thus one should not assume that all fast ODEs represent transport, without appropriate supporting information. Backtrajectories for periods of very high $\left[\mathrm{Cl}_{2}\right]$ during OASIS indicate air mass transport over the surface of the Arctic Ocean (Stephens et al., 2012). Ozone instruments onboard the OBuoy network of sea ice-tethered buoys (Knepp et al., 2010) in the Arctic Ocean have also indicated very fast ozone depletions, with a median timescale of $10.4 \mathrm{~h}$ and numerous individual events much faster than that (Halfacre et al., 2014). Unfortunately, we are limited by a lack of $\mathrm{Cl}_{2}$ measurements from across the frozen Arctic Ocean, and, thus, it is not possible to speculate how widespread this elevated $\mathrm{Cl}_{2}$ may be.

\subsection{The impact of chlorine chemistry on oxidative capacity}

One of the primary objectives of this work was to assess the impact of chlorine on bromine chemical cycles relating to ODEs in light of the recent discovery of unexpectedly high $\left[\mathrm{Cl}_{2}\right]$ in Barrow during OASIS. We discussed the contribution of observed levels of chlorine to ozone depletion in Sect. 3.2 and the impact of varying concentrations of $\mathrm{Cl}_{2}$ on the rate and timescale of ozone depletion. These results show that chlorine itself plays only a minor role in the direct destruction of ozone until $\mathrm{Cl}_{2}$ reaches over $100 \mathrm{pptv}$. That $\mathrm{Cl}$ atoms do not directly contribute significantly to ozone depletions is not surprising, given their lower ambient concentration compared to $\mathrm{Br}$ (see Fig. 2), due to the multitude of $\mathrm{Cl}$ atom sinks. In the model, the rate of $\mathrm{Cl}$ atom production from $\mathrm{Cl}_{2}$ and $\mathrm{BrCl}$ (assumed to be surface emitted species) is also on average only $20 \%$ of the rate of $\mathrm{Br}$ production from $\mathrm{Br}_{2}$ and $\mathrm{BrCl}$. The $\mathrm{Br} / \mathrm{Cl}$ ratio for the Arctic has been estimated to range from 80 to 1200 when ozone is not fully depleted (Jobson et al., 1994; Keil and Shepson, 2006; Cavender et al., 2008), based on observations of halocarbons and hydrocarbon decay. Our model predicts daytime $\mathrm{Br} / \mathrm{Cl}$ ratios for non- $\mathrm{O}_{3}$-depleted days ranging from a low of 18 up to 1300 , consistent with previous estimates. When ozone is fully depleted (i.e., <5 ppbv), [Br] becomes much greater than $[\mathrm{BrO}]$, due to the loss of its primary atmospheric sink and the resultant lack of production of $\mathrm{BrO}$, and the $\mathrm{Br} / \mathrm{Cl}$ ratio increases dramatically to $8 \times 10^{3}-2.5 \times 10^{5}$. During these periods when the ozone concentration is low, $\mathrm{CH}_{3} \mathrm{CHO}$ becomes an important $\mathrm{Br}$ atom sink, with a rate of reaction with $\mathrm{Br}$ comparable to that of $\mathrm{Br}+\mathrm{O}_{3}$ (Fig. 7). This supports the hypothesis by Shepson et al. (1996) that aldehydic compounds, including $\mathrm{CH}_{3} \mathrm{CHO}$, represent important $\mathrm{Br}$ sinks during ODEs. $\mathrm{HCHO}$ is at times a major $\mathrm{Br}$ atom sink, but only when ambient concentrations are high enough to compete with $\mathrm{CH}_{3} \mathrm{CHO}$ (e.g., towards the second half of $28 \mathrm{March}$ where $\left.[\mathrm{HCHO}] /\left[\mathrm{CH}_{3} \mathrm{CHO}\right]=\sim 0.8\right)$. Interestingly, $\mathrm{NO}_{2}$ also represents a major $\mathrm{Br}$ sink during ODEs. In comparison to other Arctic locations, Barrow can have relatively high $\mathrm{NO}_{x}$, and thus $\mathrm{NO}_{2}$ would likely not be as important a $\mathrm{Br}$ sink in more pristine Arctic environments. A detailed study into the impact of these increased $\mathrm{NO}_{x}$ levels in Barrow is the subject of Custard et al. (2015), and thus is not discussed extensively here.

Although $\mathrm{Cl}$ chemistry can generate $\mathrm{HCHO}$ and $\mathrm{CH}_{3} \mathrm{CHO}$ in the gas phase through oxidation of methane and ethane, respectively (e.g., Reactions R13-R15), it is likely not the case that these important $\mathrm{Br}$ sinks are the result of such reactions during ODEs. From the time series of ambient $\mathrm{Cl}_{2}$ observations (Fig. 1a), and as discussed in Liao et al. (2014), it is apparent that substantial mole ratios of $\mathrm{Cl}_{2}$ are only observed when $\mathrm{O}_{3}$ and radiation are present. When $\mathrm{O}_{3}$ is fully depleted during 26-28 $\mathrm{March}, \mathrm{Cl}_{2}$ is nearly absent. Moreover, the efficiency of production of $\mathrm{HCHO}$ and $\mathrm{CH}_{3} \mathrm{CHO}$ is $\mathrm{NO}_{x}$ dependent, with hydroperoxide production more important at low $\mathrm{NO}_{x}$ levels typical of more remote Arctic environments. Thus, while some significant gas-phase production of $\mathrm{HCHO}$ and $\mathrm{CH}_{3} \mathrm{CHO}$ can occur if [Cl] is high (Sumner et al., 2002), it is far more likely that in the Arctic, surface concentrations of these compounds are primarily derived from snowpack emissions (Grannas et al., 2007; Barret et al., 2011). The production of $\mathrm{HCHO}$ and $\mathrm{CH}_{3} \mathrm{CHO}$ from the snowpack has been documented in previous studies (Sumner and Shepson, 1999; Grannas et al., 2002) and strong vertical fluxes of both compounds were observed during OASIS (Barret et al., 2011; Gao et al., 2012).

As discussed previously, and shown in Fig. 6, only a small fraction of available $\mathrm{Cl}$ atoms react with $\mathrm{O}_{3}$. This is due to the very efficient reactions of $\mathrm{Cl}$ with numerous organics, with rate constants much faster than analogous oxidation by $\mathrm{OH}$. In Fig. 8, we investigate the most important $\mathrm{Cl}$ atom sinks. 


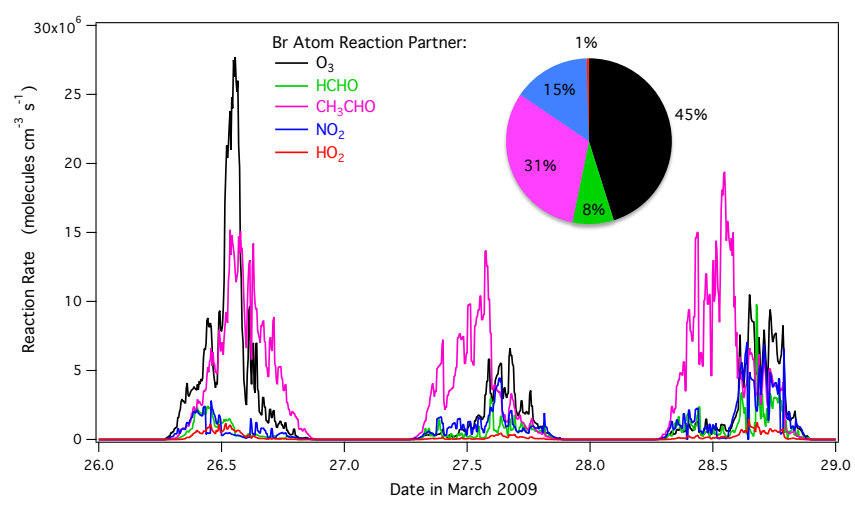

Figure 7. Time-varying rates (in molecules $\mathrm{cm}^{-3} \mathrm{~s}^{-1}$ ) of the important $\mathrm{Br}$ atom sinks during ODEs $\left(\mathrm{O}_{3}<5 \mathrm{ppbv}\right)$. The inset pie chart represents the fractional importance of each sink calculated as the median value across the 3 days shown. Time is expressed in Alaska Standard Time.

Indeed, reaction with organic compounds dominates the $\mathrm{Cl}$ atom reactivity for both non-ODE and ODE days. Reactions with reduced hydrocarbons (red trace) are the primary sink under both circumstances, at 63 and $78 \%$, respectively. When $\mathrm{O}_{3}$ is present, the $\mathrm{O}_{3}$ reaction sink accounts for $\sim 27 \%$ of $\mathrm{Cl}$ atom reaction (calculated as the median across 29 and 30 March), with oxygenated organic compounds (OVOCs) accounting for $9 \%$. When ozone is depleted, the OVOCs make up $22 \%$ of the $\mathrm{Cl}$ atom reactivity. The remaining sinks (i.e., $\mathrm{HO}_{x}, \mathrm{NO}_{y}$, and other inorganic halogen compounds) are insignificant in comparison. Thus, one of the most important impacts of $\mathrm{Cl}$ chemistry in this environment is its direct contribution to the oxidative capacity of the near-surface boundary layer via oxidation of organic compounds.

Based on the reaction scheme shown in Reactions (R13)(R15), and similar VOC oxidation pathways involving $\mathrm{Cl}$ atoms, $\mathrm{Cl}$ chemistry generates $\mathrm{HO}_{2}$ as a by-product, a very significant $\mathrm{BrO}$ sink (though relatively unimportant for $\mathrm{Br}$ atoms) and itself an important atmospheric oxidant; however, this too is dependent on $\mathrm{NO}_{x}$. It has been suggested that the presence of chlorine will significantly increase $\mathrm{HO}_{2}$ (Rudolph et al., 1999; Piot and von Glasow, 2009). Figure 9a presents results of a sensitivity study in which simulations with different combinations of halogens present were performed to investigate the impact on $\mathrm{HO}_{2}$. For days when $\mathrm{O}_{3}$ is not fully depleted (i.e., 25, 29, and 30 March), and when $\mathrm{Cl}_{2}$ is present, the " $\mathrm{Cl}$ Only" simulation results in $\mathrm{HO}_{2}$ concentrations up to $3 \times 10^{8}$ molecules cm $\mathrm{cm}^{-3}(\sim 10 \mathrm{pptv}$ ) for $\mathrm{Cl}_{2}$ nearing 100 pptv (29 and 30 March). Especially for 29 and $30 \mathrm{March}$, it is apparent that the "Br Only" simulations have much lower daytime $\mathrm{HO}_{2}$, generally remaining below $1 \times 10^{8}$ molecules $\mathrm{cm}^{-3}$. When $\mathrm{Cl}_{2}$ is present (i.e., when $\mathrm{O}_{3}$ is not depleted), the " $\mathrm{Cl}$ Only" simulation is nearly indistinguishable from the Base Model, indicating the chlorine is dominating the halogen contribution to $\mathrm{HO}_{2}$. However, in some cases, specifically when $\mathrm{O}_{3}$ is depleted (and thus $\mathrm{Cl}_{2}$ is

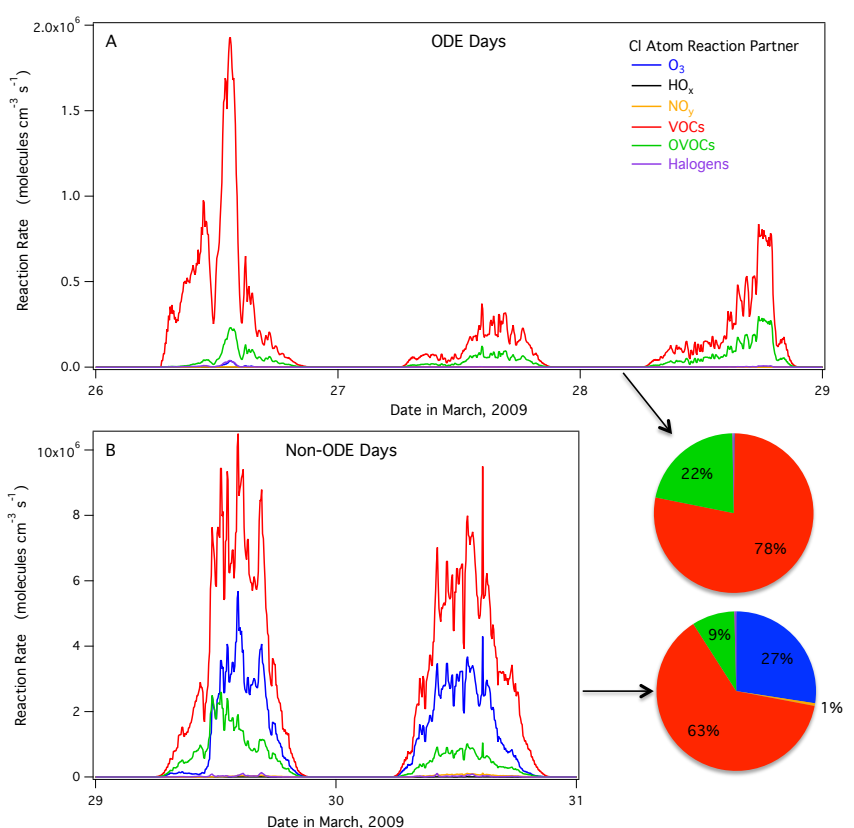

Figure 8. Rates of reaction for the most important $\mathrm{Cl}$ atom reaction partners during ODE days (a; $\mathrm{O}_{3}>5$ ppbv) and non-ODE days (b). The corresponding pie charts represent the fractional importance of each sink, calculated as median values across the days shown. $\mathrm{HO}_{x}$ is defined as the sum of $\mathrm{OH}$ and $\mathrm{HO}_{2}, \mathrm{NO}_{y}$ is the sum of all nitrogen oxide compounds, VOCs include all non-functionalized alkanes, alkenes, and acetylene, OVOCs include all non-radical organic compounds with an oxygen (carbonyls, ketones, carboxylic acids), and halogens include all inorganic halogenated species.

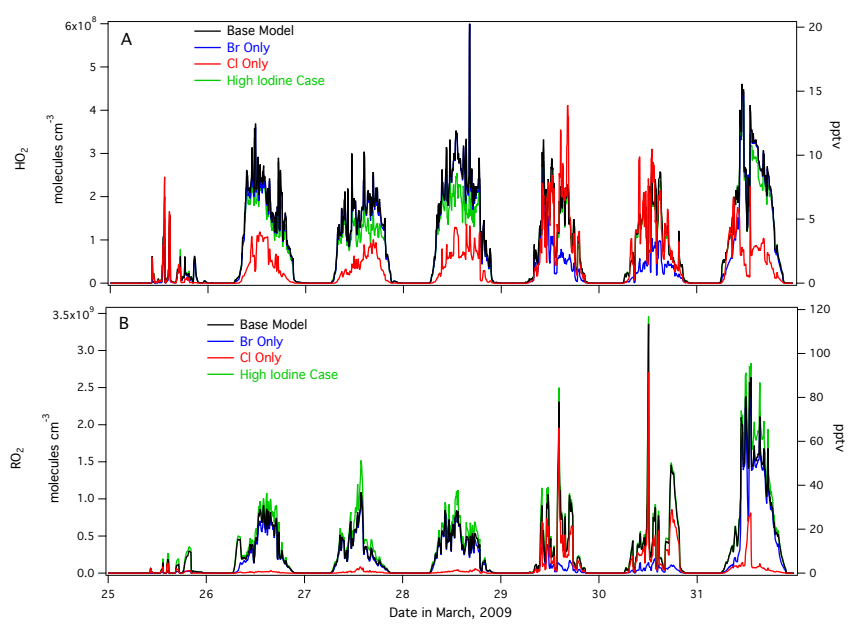

Figure 9. Comparison of modeled $\mathrm{HO}_{2}$ (a) and $\mathrm{RO}_{2}$ (b) from four different modeling scenarios: $\mathrm{Base}(\mathrm{Br}$ and $\mathrm{Cl}$ present; black trace), Br Only (blue trace), $\mathrm{Cl}$ Only (red trace), and Base + High Iodine (green trace). $\mathrm{RO}_{2}$ is defined here as the sum of the methyl through butyl forms of alkyl peroxy radicals. Time is expressed in Alaska Standard Time. 
Table 10. Partitioning of the $\mathrm{HO}_{x}, \mathrm{BrO}_{x}, \mathrm{ClO}_{x}$, and $\mathrm{IO}_{x}$ radical partners for mid-day of 29 March for a variety of modeling scenarios performed by including or excluding different halogen species.

\begin{tabular}{lrrrr}
\hline Model conditions & $\begin{array}{r}{\left[\mathrm{HO}_{2}\right] \backslash[\mathrm{OH}]} \\
\text { ratio }\end{array}$ & $\begin{array}{r}{[\mathrm{BrO}] \backslash[\mathrm{Br}]} \\
\text { ratio }\end{array}$ & $\begin{array}{r}{[\mathrm{ClO}] \backslash[\mathrm{Cl}]} \\
\text { ratio }\end{array}$ & $\begin{array}{r}{[\mathrm{IO}] \backslash[\mathrm{I}]} \\
\text { ratio }\end{array}$ \\
\hline No Halogens & 115 & - & - & - \\
Br Only & 63.0 & 7.65 & - & - \\
Cl Only & 229 & - & 293 & - \\
Low Iodine Only & 99.4 & - & - & 2.60 \\
High Iodine Only & 62.5 & - & - & 2.30 \\
Br and Cl (Base) & 104 & 5.66 & 247 & - \\
Br and Low Iodine & 60.8 & 7.34 & - & 1.96 \\
Br and High Iodine & 53.1 & 6.12 & - & 1.76 \\
$\mathrm{Br}, \mathrm{Cl}$, and Low Iodine & 98.7 & 5.48 & 244 & 1.74 \\
Br, Cl, and High Iodine & 81.0 & 4.68 & 230 & 1.57 \\
\hline
\end{tabular}

absent as discussed above), $\mathrm{HO}_{2}$ is indistinguishable from a "Br Only" case. During the 26-28 March period, there would be little $\mathrm{HO}_{x}$ production from $\mathrm{O}_{3}$ photolysis, and as $\mathrm{Cl}_{2}$ is also nearly absent, bromine chemistry and photolysis of $\mathrm{HOx}$ precursors from the snowpack (e.g., HCHO or HONO) become the primary sources of $\mathrm{HO}_{x}$ production.

The production of $\mathrm{HO}_{2}$ as a result of chlorine chemistry is also apparent when examining the $\left[\mathrm{HO}_{2}\right] /[\mathrm{OH}] \mathrm{ra}-$ tio in simulations performed with and without chlorine. Table 10 presents $\left[\mathrm{HO}_{2}\right] \backslash[\mathrm{OH}],[\mathrm{BrO}] \backslash[\mathrm{Br}],[\mathrm{ClO}] \backslash[\mathrm{Cl}]$, and $[\mathrm{IO}] \backslash[\mathrm{I}]$ ratios, calculated for mid-day of $29 \mathrm{March}$, for a variety of model scenarios with no halogen chemistry and different combinations of halogens present. When halogen chemistry was excluded from the model, the $\left[\mathrm{HO}_{2}\right] \backslash[\mathrm{OH}]$ ratio was 115 ; this nearly doubled to 230 when only chlorine chemistry was added. This effect is in contrast to the " $\mathrm{Br}$ Only", "Low Iodine Only", and "High Iodine Only" simulations, where the addition of bromine or iodine served to decrease the $\left[\mathrm{HO}_{2}\right] /[\mathrm{OH}]$ ratio. Futhermore, the addition of chlorine to any scenario shifted this ratio towards $\mathrm{HO}_{2}$. For example, "Br Only" decreased this ratio from 115 to 63; upon the addition of chlorine for the " $\mathrm{Br}$ and $\mathrm{Cl}$ (Base)" scenario, the ratio was increased to 104. It is important to note that $\mathrm{NO}_{x}$ chemistry has a very significant impact on both $\mathrm{HO}_{x}$ and halogen chemistry. For these calculations, $\mathrm{NO}$ and $\mathrm{NO}_{2}$ were held constant (at 25 and 50 pptv, respectively) so that we could investigate the impacts of just adding or removing halogens without concomitant changes in $\mathrm{NO}_{x}$. If $\mathrm{NO}_{x}$ is not constrained, the removal of halogens has the effect of leading to increased $\mathrm{NO}_{x}$ levels (through removal of this sink), which then decreases $\mathrm{HO}_{x}$. This leads to a confounding influence on the $\mathrm{HO}_{x}$ ratio that is due to increased $\mathrm{NO}_{x}$ chemistry rather than removal of halogens.

To investigate further, time-varying rates of production of $\mathrm{OH}$ and $\mathrm{HO}_{2}$ were determined from their primary source reactions (Fig. 10). Under our modeling conditions for the 7 days studied, when $\mathrm{O}_{3}$ is not fully depleted, $\mathrm{HO}_{x}$ is produced primarily through photolysis of $\mathrm{HONO}$ (as postulated

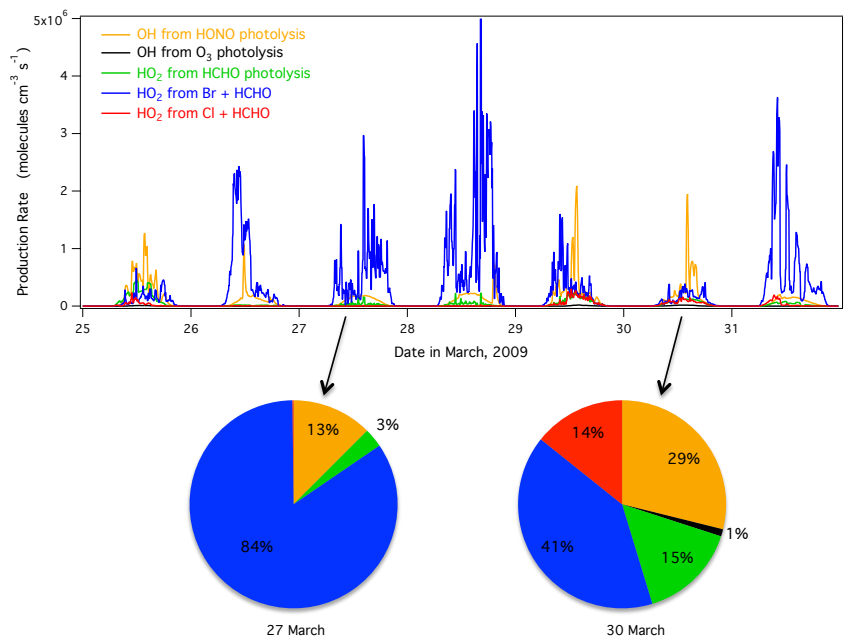

Figure 10. Comparison of $\mathrm{HO}_{x}$ production rates (in molecules $\mathrm{cm}^{-3} \mathrm{~s}^{-1}$ ) for five primary pathways: $\mathrm{OH}$ production from HONO photolysis (orange trace), $\mathrm{OH}$ production from $\mathrm{O}_{3}$ photolysis and subsequent reaction with $\mathrm{H}_{2} \mathrm{O}$ (black trace), $\mathrm{HO}_{2}$ production from $\mathrm{HCHO}$ photolysis (green trace), $\mathrm{HO}_{2}$ production from $\mathrm{Br}+\mathrm{HCHO}$ reaction (blue trace), and $\mathrm{HO}_{2}$ production from $\mathrm{Cl}+\mathrm{HCHO}$ reaction (red trace). The corresponding pie charts represent the fractional importance of each source for a select ODE day (27 March; $\mathrm{O}_{3}>5$ ppbv) and a select non-ODE day (30 March) calculated as the median value across each day. Time is expressed in Alaska Standard Time.

by Zhou et al., 2001, and Villena et al., 2011) and as a product of $\mathrm{Br}$ reaction with $\mathrm{HCHO}$ (orange and blue traces in Fig. 8, 29 and $41 \%$, respectively, for 30 March), with significant production from photolysis of $\mathrm{HCHO}(15 \%$ for $30 \mathrm{March})$ as well, confirming the importance of snowpack-emitted carbonyl compounds for the oxidation capacity of the Arctic boundary layer (Sumner and Shepson, 1999). When $\mathrm{O}_{3}$ is fully depleted, $\mathrm{HCHO}$ is the primary direct $\mathrm{HO}_{x}$ source via reaction with $\mathrm{Br}$ ( $84 \%$ for $27 \mathrm{March})$. This result explains the observation in Fig. 8a that on $\mathrm{O}_{3}$-depleted days, the " $\mathrm{Br}$ 
Only" and Base simulations give essentially identical results for $\left[\mathrm{HO}_{2}\right]$. Although these modeling results clearly indicate that $\mathrm{Br}$ oxidation is the dominant $\mathrm{HO}_{2}$ source during ODEs, the absolute value of the $\mathrm{HO}_{2}$ production from this pathway during the ozone-depleted days should be regarded as an upper limit, since, as explained previously, the uncertainty in the $\mathrm{BrO}$ measurements is high during this time and it is possible that $[\mathrm{Br}]$ is somewhat overestimated. Indeed, this is likely the reason behind the high bias of the model $\mathrm{HO}_{2}$ compared to measurements for 26-28 March (Fig. 2g).

Finally, an additional significant impact of chlorine chemistry on the oxidation capacity of the polar boundary layer (PBL) is the production of $\mathrm{RO}_{2}$ through Reaction (R13) and analogous oxidation reactions with higher order hydrocarbons. $\mathrm{RO}_{2}$ reaction with $\mathrm{BrO}$ can also lead to production of $\mathrm{HOBr}$ through Reaction (R19), which could enhance the heterogeneous production of $\mathrm{Br}$ through $\mathrm{HOBr}$ deposition and the bromine explosion mechanism.

$\mathrm{BrO}+\mathrm{R}-\mathrm{CH}_{2} \mathrm{OO} \rightarrow \mathrm{HOBr}+\mathrm{R}-\mathrm{CHOO}$

However, the presence of chlorine chemistry also decreases the $[\mathrm{BrO}] /[\mathrm{Br}]$ ratio through the $\mathrm{BrO}+\mathrm{ClO}$ reaction, so $\mathrm{Re}-$ action (R19) may affect [HOBr] most under certain chemical conditions (e.g., lower $[\mathrm{ClO}]$ ). $\left[\mathrm{RO}_{2}\right]$ (calculated as the sum of methyl through butyl forms) for simulations conducted with and without chlorine is shown in Fig. 9b. This figure illustrates that when $\mathrm{Cl}_{2}$ is high, i.e., on 25,29 , and 30 March, there is sometimes a large impact on $\mathrm{RO}_{2}$, as can by seen by comparing the "Br Only" case (blue trace) with the Base Model (black trace). Again, $\left[\mathrm{Cl}_{2}\right]$ is very low when ozone is depleted, so simulated $\left[\mathrm{RO}_{2}\right]$ during ozone-depleted days becomes nearly indistinguishable for the Base and "Br Only" scenarios, as was shown already for $\mathrm{HO}_{2}$. These results support the hypothesis that $\mathrm{Cl}$ can substantially increase the oxidation capacity of the Arctic troposphere when present at elevated mixing ratios. The levels of $\mathrm{RO}_{2}$ that are present when $\mathrm{Cl}$ is not included in the model are primarily a result of both $\mathrm{Br}$ - and $\mathrm{OH}$-oxidation chemistry; the $\mathrm{RO}_{2}$ spike on 31 March that is seen in the " $\mathrm{Br}$ Only' simulation, for example, is a result of the observed spike in $\mathrm{CH}_{3} \mathrm{CHO}$ and reaction with $\mathrm{Br}$. The impact of the increased $\mathrm{RO}_{2}$ due to $\mathrm{Cl}$ chemistry appears to be less significant as a $\mathrm{BrO}$ sink, however, as $\mathrm{CH}_{3} \mathrm{OO}$ (the dominant $\mathrm{RO}_{2}$ species) represents only a minor sink for $\mathrm{BrO}$ (Thompson et al., 2015), and $\mathrm{BrO}$ is likewise a minor sink for $\mathrm{CH}_{3} \mathrm{OO}$ in comparison to $\mathrm{HO}_{2}$ and $\mathrm{NO}$ (the median sink contribution of $\mathrm{BrO}$ to $\mathrm{CH}_{3} \mathrm{OO}$ is $0.05 \%$ ).

Based on our analysis, it appears possible that the presence of chlorine can promote the production of reactive bromine species through two distinct mechanisms: (1) oxidation chemistry due to chlorine, which under certain chemical conditions can (e.g., high $\mathrm{Cl}_{2}$, elevated $\mathrm{NO}_{x}$ ) increase $\left[\mathrm{HO}_{2}\right]$ and/or $\left[\mathrm{RO}_{2}\right]$, thereby increasing the production of $\mathrm{HOBr}$, and thus, the heterogeneous recycling of bromine, and (2) gas-phase $\mathrm{HOCl}$ can react with $\mathrm{Br}^{-}$in the aqueous phase to produce $\mathrm{BrCl}$, thus producing reactive bromine (though this pathway is not as significant for $\mathrm{Br}$ atom production as is $\mathrm{Br}_{2}$ ). Conversely, the presence of both chloride and bromide ions in the aqueous phase can lead to a competition between the production of $\mathrm{Br}_{2}, \mathrm{BrCl}$, or $\mathrm{Cl}_{2}$. When we initially ran our model with only heterogeneous production of molecular halogens, large increases in gas-phase $[\mathrm{Br}]$ were produced when aerosol chloride was decreased by 3 orders of magnitude, but no difference in $[\mathrm{Br}]$ occurred when snowpackphase chloride was decreased by an equivalent amount. The snowpack is by far the primary source of both $\mathrm{Br}$ and $\mathrm{Cl}$ atom precursors in our model, as shown in Michalowski et al. (2000); however, the greater sensitivity to the aerosol chloride loading suggests that the aerosol could be a potentially important source of $\mathrm{Cl}$ atom precursors to the atmosphere. This may likely be related to the aerosol $\mathrm{pH}$. Laboratory and field studies would be required to test this hypothesis. It is clear that the interactions between bromine and chlorine are quite complex in that chlorine chemistry can produce reactive bromine sinks (when at elevated concentrations), but the presence of chlorine can also increase $\mathrm{Br}$ atom production under certain circumstances, thereby increasing the rate of ozone depletion in an indirect fashion.

\subsection{The impact of iodine chemistry and bromine-iodine interactions}

Several modeling studies have also investigated the potential impact of iodine on ozone depletion in the troposphere (e.g., Chameides and Davis, 1980; Davis et al., 1996; Sander et al., 1997; Calvert and Lindberg, 2004b; Saiz-Lopez et al., 2008), in each case concluding that it could be very important. Iodine is potentially one of the most important species in Arctic ozone chemistry, and yet there is very little observational information. To date, no conclusive measurements of IO in the High Arctic have been achieved, though recently our group has detected substantial concentrations of $I_{2}$ in the snowpack air in Barrow with CIMS (Raso et al., 2015); thus, there is evidence that iodine chemistry is present to some extent.

As discussed in Sect. 3.2, iodine chemistry can greatly enhance the rate of ozone depletion, both through the direct reaction of I with ozone (given that ozone is the primary I atom sink, Fig. 4) and through the indirect effect of increasing available $\mathrm{Br}$ atoms through the cross-reaction of $\mathrm{IO}$ and BrO. This can be seen in Table 10, where it is shown that the $[\mathrm{BrO}] /[\mathrm{Br}]$ ratio is decreased from 7.65 in the "Br Only" simulation to 7.34 or 6.12 upon addition of low iodine or high iodine levels, respectively. In a similar fashion, iodine chemistry also shifts the $\mathrm{ClO}_{x}$ partitioning, decreasing the midday $[\mathrm{ClO}] \backslash[\mathrm{Cl}]$ for 29 March from 247 in the Base Model $(\mathrm{Br}$ and $\mathrm{Cl})$ to 244 or 230 when low or high levels are iodine are present, respectively, due to the $\mathrm{IO}+\mathrm{ClO}$ reaction and decreased $\left[\mathrm{O}_{3}\right]$. The decreased $[\mathrm{ClO}] \backslash[\mathrm{Cl}]$ ratio could then impact the radical distribution through $\mathrm{RO}_{2}$ production. The $[\mathrm{IO}] \backslash[\mathrm{I}]$ ratio is much lower than either the $\mathrm{BrO}_{x}$ or $\mathrm{ClO}_{x}$ ratio due to fast photolysis of IO and far fewer known I atom 
sinks (Vogt, 1999). Under the conditions employed here on 29 March, the $[\mathrm{IO}] \backslash[\mathrm{I}]$ ratio ranges from 1.57 to 2.60 .

Previous modeling studies have concluded that iodine chemistry can impact the partitioning of important atmospheric oxidants by decreasing the $\left[\mathrm{HO}_{2}\right] \backslash[\mathrm{OH}]$ ratio through the formation of $\mathrm{HOI}$ and subsequent photolysis. The rate constant for the $\mathrm{IO}+\mathrm{HO}_{2}$ reaction is a factor of $\sim 2.5$ and 10 faster than the analogous $\mathrm{BrO}$ and $\mathrm{ClO}$ reactions, respectively; thus, iodine has the potential to have a significant impact on the $\left[\mathrm{HO}_{2}\right] \backslash[\mathrm{OH}]$ ratio, and $\mathrm{HO}_{2}$ can represent a primary IO sink during periods of low [IO] and [BrO] (Vogt, 1999). As mentioned above and shown in Table 10 , the $\left[\mathrm{HO}_{2}\right] \backslash[\mathrm{OH}]$ ratio at mid-day on 29 March with no halogens present is 115 . The presence of bromine alone decreases this ratio to 63 , and inclusion of iodine further decreases this to 60.8 or 53.1 when at low or high levels, respectively. There of course can be wide variability in the absolute ratios depending on specific chemical conditions, such as $\mathrm{NO}_{x}$ or VOC levels. Saiz-Lopez et al. (2007b) determined a $\left[\mathrm{HO}_{2}\right] \backslash[\mathrm{OH}]$ ratio of 33 due to bromine-iodine chemistry in Antarctica.

The decrease in the $\mathrm{ClO}_{x}$ ratio effected by iodine could also impact the oxidizing capacity of the PBL. In fact, we see a slight increase in $\left[\mathrm{RO}_{2}\right]$ when iodine is present at high levels in the multiday model, with a greater increase during $\mathrm{O}_{3}$ depleted days when iodine is included (green trace, Fig. 9b). Because iodine atoms do not oxidize VOCs, the increased $\mathrm{RO}_{2}$ is likely a result of the combined effects of a shift in the $\mathrm{ClO}_{x}$ ratio toward $\mathrm{Cl}$, and a shift in the $\mathrm{HO}_{x}$ ratio toward $\mathrm{OH}$, resulting in a decrease in $\mathrm{RO}_{2}$ sinks and an increase in production. These impacts suggest an important indirect role for iodine in mediating the oxidation potential of the PBL, in the presence of the other halogens.

We note, however, that the observed $\left[\mathrm{Cl}_{2}\right]$ and $\left[\mathrm{Br}_{2}\right]$ are lower during ozone-depleted days than during non-ozonedepleted days. This suggests a surface activation mechanism controlled by $\mathrm{O}_{3}$, presumably in part through a snowpack halogen explosion mechanism, such as has been observed in laboratory studies by, e.g., Oum et al. (1998a, b) and the recent Pratt et al. (2013) study using natural Barrow snow. In our model, because the production mechanism of $\mathrm{I}_{2}$ is undefined and we have no observations with which to compare, the $\mathrm{I}_{2}$ flux is of the same magnitude each day, independent of $\left[\mathrm{O}_{3}\right]$. Therefore, during the $\mathrm{O}_{3}$-depleted days when $\left[\mathrm{Br}_{2}\right]$ and $\left[\mathrm{Cl}_{2}\right]$ are much reduced, iodine chemistry becomes dramatically more important in relative terms. However, laboratory studies have indicated that $\mathrm{I}_{2}$ can be produced via $\mathrm{O}_{3}$ oxidation of aqueous iodide (Garland and Curtis, 1981; Martino et al., 2009; Carpenter et al., 2013). If it were indeed the case that $\mathrm{I}_{2}$ is also produced through an $\mathrm{O}_{3}$-mediated activation mechanism, this would likely eliminate the large difference between depleted and non-depleted days that is seen here. More studies are required to determine the dominant mechanism and kinetics for the heterogeneous surface production of $\mathrm{I}_{2}$.
As discussed previously, the calculations in Table 10 were performed by fixing $\mathrm{NO}$ and $\mathrm{NO}_{2}$ concentrations so that we could investigate halogen impacts on $\mathrm{HO}_{x}$. However, we did examine the impact of halogen chemistry on the $\left[\mathrm{NO}_{2}\right] \backslash[\mathrm{NO}]$ ratio. This was performed simply by initiating the model with $\mathrm{NO}$ and $\mathrm{NO}_{2}$ mole ratios of 25 and $50 \mathrm{pptv}$, respectively, and then allowing them to freely evolve. For these sensitivity studies, the presence of halogen chemistry in our model increased the $\left[\mathrm{NO}_{2}\right] \backslash[\mathrm{NO}]$ ratio, primarily through Reaction (R16), though $\mathrm{Cl}$ chemistry can further increase this ratio through the production of $\mathrm{RO}_{2}$ (e.g., Reactions R13-R14). However, though bromine increases the mid-day $\mathrm{NO}_{x}$ ratio from 0.68 to 1.81 for 29 March compared to a model run conducted with no halogens, the further addition of low iodine only increases it to 1.85 . The absolute concentrations of $\left[\mathrm{NO}_{2}\right]$ and $[\mathrm{NO}]$ both decrease with the addition of halogens, but the $\left[\mathrm{NO}_{2}\right] \backslash[\mathrm{NO}]$ ratio increases because $\mathrm{XO}$ reaction with $\mathrm{NO}$ is generally faster than reaction with $\mathrm{NO}_{2}$. However, in the case of iodine, IO reaction with $\mathrm{NO}_{2}$ is faster than its reaction with $\mathrm{NO}$, and I atoms also react very efficiently with $\mathrm{NO}_{2}$. Thus, chlorine should have a greater effect on increasing the $\mathrm{NO}_{x}$ ratio than iodine. Indeed, when the model was run with bromine and chlorine (the Base scenario), the $\left[\mathrm{NO}_{2}\right] \backslash[\mathrm{NO}]$ ratio significantly increased to 8.4 .

\section{Conclusions}

The goal of this work was to investigate the interactions and impacts of halogen chemistry on ozone depletion using a model that is constrained to a unique set of observed, timevarying chemical conditions at the time of the event. With this approach, we have been able to dissect some of the important chemical pathways pertaining to ODEs, focusing on the interactions between the halogen radicals. It is clear that the interactions between bromine, chlorine, and iodine are very complex and highly dependent on the concurrent conditions of relevant species, such as $\mathrm{O}_{3}, \mathrm{HO}_{x}, \mathrm{NO}_{x}$, and the VOCs. As these species fluctuate, the partitioning of halogen species will also change, and so too will their impact on chemistry of the PBL. Thus, a full understanding of halogen chemistry requires the careful measurement of all these species (including $\mathrm{Cl}_{2}, \mathrm{Br}_{2}, \mathrm{HOBr}$, and $\mathrm{HOCl}$ ).

This work has demonstrated that bromine chemistry is clearly the dominant destruction pathway for ozone depletion episodes, but that chlorine and, especially, iodine, can contribute significantly to both the rate and timescale of ozone depletion. The new observations of high chlorine levels at Barrow potentially change the way we view ODEs. Often, observations of rapid decline in ozone at various locations have been attributed to transport because bromine chemistry alone was not enough to account for the ozone depletion rate (Hausmann and Platt, 1994; Tuckermann et al., 1997; Bottenheim et al., 2002). As we have shown, complete ozone 
depletions can occur on timescales of much less than a day, and at elevated chlorine levels, even as short as several hours. This result suggests that more of the ODEs that are observed could be the result of local-scale chemistry; however, as noted previously, our calculated timescales for ozone depletion are based upon measurements conducted within the near-surface layer, and may not necessarily reflect the overall depletion timescale through the boundary layer. While chlorine is clearly not necessary to cause ozone depletion, it can impact the rate of ozone depletion through synergistic effects of cross-reactions that enhance $\mathrm{Br}$ atom recycling. Moreover, the presence of elevated chlorine levels can impact important Arctic chemical budgets, including $\mathrm{HO}_{x}, \mathrm{NO}_{x}$, and VOCs, with implications for the oxidative capacity of the PBL. More field measurements, including over the frozen Arctic Ocean, are necessary to evaluate the ubiquity of these elevated chlorine levels. In light of these new data, it is crucial that future Arctic modeling studies take into account the activity of chlorine.

In our model, we prescribed a $\mathrm{Cl}_{2}$ mixing ratio that was varied from 25 to $400 \mathrm{pptv}$ for 29 March in sensitivity studies to investigate the impact of elevated $\mathrm{Cl}_{2}$. However, we also determined the $\mathrm{Cl}_{2}$ fluxes that would be necessary to produce the desired gas-phase $\left[\mathrm{Cl}_{2}\right]$. In our model, volumetric fluxes ranging from $5.5 \times 10^{5}$ molecules $\mathrm{cm}^{-3} \mathrm{~s}^{-1}$ to $1.45 \times 10^{6}$ molecules $\mathrm{cm}^{-3} \mathrm{~s}^{-1}$ were required. If we assume a boundary layer mixing height of $300 \mathrm{~m}$, the corresponding surface fluxes would be $1.65 \times 10^{10}-4.35 \times 10^{10}$ molecules $\mathrm{cm}^{-2} \mathrm{~s}^{-1}$. Similarly, the fluxes necessary for $\mathrm{Br}_{2}$ range from $1.0 \times 10^{3}$ to $9.0 \times 10^{6}$ molecules $\mathrm{cm}^{-3} \mathrm{~s}^{-1}$. These numbers are consistent with those used by Piot and von Glasow (2009). However, there are no $\mathrm{Br}_{2}$ or $\mathrm{Cl}_{2}$ measurements aloft of the surface in the Arctic. Utilizing the method of Guimbaud et al. (2002), the effective mixing height, $Z^{*}$, for $\mathrm{Cl}_{2}$ at mid-day is only $2.15 \mathrm{~m}$, making the calculated $\mathrm{Cl}_{2}$ fluxes $1.2 \times 10^{8}-3.1 \times 10^{8}$ molecules $\mathrm{cm}^{-2} \mathrm{~s}^{-1} ; Z^{*}$ for $\mathrm{Br}_{2}$ is $0.5 \mathrm{~m}$, leading to a calculated $\mathrm{Br}_{2}$ flux of $5.0 \times 10^{4}-$ $4.5 \times 10^{8}$ molecules $\mathrm{cm}^{-2} \mathrm{~s}^{-1}$. Therefore, field studies aimed at determining the magnitude of the surface flux of $\mathrm{Cl}_{2}$ and $\mathrm{Br}_{2}$ are warranted. This disparity also points out the importance of measurements of the vertical profiles of molecular halogens above the snowpack/sea ice surface.

Finally, we find that iodine could be the most efficient halogen for depleting ozone on a per atom basis, given our assumed fluxes. We assume very low [IO] in our model $(\leq 1 \mathrm{pptv})$, below the detection limit of the DOAS instrumentation, and yet the resulting enhancements in ozone depletion are quite significant. Higher levels of iodine would certainly have a dramatic effect on ozone chemistry, as illustrated by our simulations incorporating $0.5 \mathrm{pptv} \mathrm{I}_{2}(\sim 10 \mathrm{pptv} \mathrm{IO})$. A possible mechanism for the production of $\mathrm{I}_{2}$ in the polar regions is the activity of ice algae and phytoplankton that produce iodine compounds, which are then wicked to the surface through brine channels (Mahajan et al., 2010). It has also been suggested that the primary productivity in Antarctic ice and waters may be higher than in the Arctic (Arrigo et al., 1997; Gosselin et al., 1997; Lizotte, 2001; Mahajan et al., 2010), possibly accounting for the difference in apparent iodine activity between the two poles. However, as the ice in the Arctic continues to thin, and as more multiyear ice has been replaced by seasonal sea ice (Nghiem et al., 2012), the algae and phytoplankton productivity in the Arctic has increased (Arrigo et al., 2008, 2012). This could lead to an increase in iodine emissions in the future, and thus to a greater occurrence of springtime ODEs. The further development of analytical methods capable of measuring the very low [IO] and $\left[\mathrm{I}_{2}\right]$ potentially present in the Arctic should be a high priority for the further advancement of this research.

Acknowledgements. The modeling analysis presented herein was funded by National Science Foundation grant ARC-0732556. Partial support for CT during preparation of this manuscript was provided by the NSF Atmospheric and Geospace Sciences Postdoctoral Research Fellowship program. The authors wish to thank the organizers of the OASIS 2009 field campaign, the Barrow Arctic Science Consortium for logistics support, and all of the researchers who contributed to the campaign.

Edited by: A. Dastoor

\section{References}

Adams, J. W., Holmes, N. S., and Crowley, J. N.: Uptake and reaction of $\mathrm{HOBr}$ on frozen and dry $\mathrm{NaCl} / \mathrm{NaBr}$ surfaces between 253 and 233 K, Atmos. Chem. Phys., 2, 79-91, doi:10.5194/acp2-79-2002, 2002.

Apel, E. C., Emmons, L. K., Karl, T., Flocke, F., Hills, A. J., Madronich, S., Lee-Taylor, J., Fried, A., Weibring, P., Walega, J., Richter, D., Tie, X., Mauldin, L., Campos, T., Weinheimer, A., Knapp, D., Sive, B., Kleinman, L., Springston, S., Zaveri, R., Ortega, J., Voss, P., Blake, D., Baker, A., Warneke, C., Welsh-Bon, D., de Gouw, J., Zheng, J., Zhang, R., Rudolph, J., Junkermann, W., and Riemer, D. D.: Chemical evolution of volatile organic compounds in the outflow of the Mexico City Metropolitan area, Atmos. Chem. Phys., 10, 2353-2375, doi:10.5194/acp-10-23532010, 2010.

Aranda, A., Le Bras, G., La Verdet, G., and Poulet, G.: The BrO+ $\mathrm{CH}_{3} \mathrm{O}_{2}$ reaction: Kinetics and role in the atmospheric ozone budget, Geophys. Res. Lett., 24, 2745-2748, 1997.

Ariya, P., Jobson, B., Sander, R., Niki, H., Harris, G., Hopper, J., and Anlauf, K.: Measurements of C2-C7 hydrocarbons during the Polar Sunrise Experiment 1994: Further evidence for halogen chemistry in the troposphere, J. Geophys. Res., 103, 1316913180, doi:10.1029/98JD00284, 1998.

Arrigo, K. R., Worthen, D. L., Lizotte, M. P., Dixon, P., and Dieckmann, G.: Primary production in Antarctic sea ice, Science, 276, 394-397, 1997.

Arrigo, K. R., van Dijken, G., and Pabi, S.: Impact of a shrinking Arctic ice cover on marine primary production, Geophys. Res. Lett, 35, L19603, doi:10.1029/2008GL035028, 2008. 
Arrigo, K. R., Perovich, D. K., Pickart, R. S., Brown, Z. W., van Dijken, G. L., Lowry, K. E., Mills, M. M., Palmer, M. A., Balch, W. M., Bahr, F., Bates, N. R., Benitez-Nelson, C., Bowler, B., Brownlee, E., Ehn, J. K., Frey, K. E., Garley, R., Laney, S. R., Lubelczyk, L., Mathis, J., Matsuoka, A., Mitchell, B. G., Moore, G. W. K., Ortega-Retuerta, E., Pal, S., Polashenski, C. M., Reynolds, R. A., Schieber, B., Sosik, H. M., Stephens, M., and Swift, J. H.: Massive Phytoplankton Blooms Under Arctic Sea Ice, Science, 336, 1408, doi:10.1126/science.1215065, 2012.

Atkinson, R., Baulch, D. L., Cox, R. A., Crowley, J. N., Hampson, R. F., Hynes, R. G., Jenkin, M. E., Rossi, M. J., and Troe, J.: Evaluated kinetic and photochemical data for atmospheric chemistry: Volume $\mathrm{I}$ - gas phase reactions of $\mathrm{O}_{x}, \mathrm{HO}_{x}, \mathrm{NO}_{x}$ and $\mathrm{SO}_{x}$ species, Atmos. Chem. Phys., 4, 1461-1738, doi:10.5194/acp-41461-2004, 2004.

Barret, M., Domine, F., Houdier, S., Gallet, J. C., Weibring, P., Walega, J., Fried, A., and Richter, D.: Formaldehyde in the Alaskan Arctic snowpack: Partitioning and physical processes involved in air-snow exchanges, J. Geophys. Res., 116, D00R03, doi:10.1029/2011JD016038, 2011.

Barrie, L., Bottenheim, J., Schnell, R., Crutzen, P., and Rasmussen, R.: Ozone destruction and photochemical reactions at polar sunrise in the lower Arctic atmosphere, Nature, 334, 138-141, 1988.

Barrie, L., Staebler, R., Toom, D., Georgi, B., Den Hartog, G., Landsberger, S., and Wu, D.: Arctic aerosol size-segregated chemical observations in relation to ozone depletion during Polar Sunrise Experiment 1992, J. Geophys. Res., 99, 25439-25451, 1994.

Beckwith, R. C., Wang, T. X., and Margerum, D. W.: Equilibrium and kinetics of bromine hydrolysis, Inorg. Chem., 35, 995-1000, 1996.

Beine, H. J., Honrath, R. E., Dominé, F., Simpson, W. R., and Fuentes, J. D.: $\mathrm{NO}_{x}$ during background and ozone depletion periods at Alert: Fluxes above the snow surface, J. Geophys. Res, 107, 101029, doi:10.1029/2002JD002082, 2002.

Bottenheim, J. W. and Chan, E.: A trajectory study into the origin of spring time Arctic boundary layer ozone depletion, J. Geophysical Res., 111, D19301, doi:10.1029/2006JD007055, 2006.

Bottenheim, J. W., Barrie, L. A., Atlas, E., Heidt, L. E., Niki, H., Rasmussen, R. A., and Shepson, P. B.: Depletion of lower tropospheric ozone during Arctic spring: The Polar Sunrise Experiment 1988, J. Geophys. Res, 95, 555-518, 1990.

Bottenheim, J. W., Fuentes, J. D., Tarasick, D. W., and Anlauf, K. G.: Ozone in the Arctic lower troposphere during winter and spring 2000 (ALERT2000), Atmos. Environ., 36, 2535-2544, 2002.

Boudries, H. and Bottenheim, J.: $\mathrm{Cl}$ and $\mathrm{Br}$ atom concentrations during a surface boundary layer ozone depletion event in the Canadian high Arctic, Geophys. Res. Lett., 27, 517-520, 2000.

Calvert, J. and Lindberg, S.: A modeling study of the mechanism of the halogen-ozone-mercury homogeneous reactions in the troposphere during the polar spring, Atmos. Environ., 37, 4467-4481, 2003.

Calvert, J. G. and Lindberg, S. E.: The potential influence of iodinecontaining compounds on the chemistry of the troposphere in the polar spring. II. Mercury depletion, Atmos. Environ., 38, 51055116, 2004a.

Calvert, J. G. and Lindberg, S. E.: Potential influence of iodinecontaining compounds on the chemistry of the troposphere in the polar spring. I. Ozone depletion, Atmos. Environ., 38, 50875104, 2004b.

Carpenter, L., Sturges, W., Penkett, S., Liss, P., Alicke, B., Hebestreit, K., and Platt, U.: Short-lived alkyl iodides and bromides at Mace Head, Ireland: Links to biogenic sources and halogen oxide production, J. Geophys. Res., 104, 1679-1689, 1999.

Carpenter, L. J., MacDonald, S. M., Shaw, M. D., Kumar, R., Saunders, R. W., Parthipan, R., Wilson, J., and Plane, J. M.: Atmospheric iodine levels influenced by sea surface emissions of inorganic iodine, Nat. Geosci., 6, 108-111, 2013.

Cavender, A. E., Biesenthal, T. A., Bottenheim, J. W., and Shepson, P. B.: Volatile organic compound ratios as probes of halogen atom chemistry in the Arctic, Atmos. Chem. Phys., 8, 17371750, doi:10.5194/acp-8-1737-2008, 2008.

Chameides, W. and Davis, D.: Iodine: Its possible role in tropospheric photochemistry, J. Geophys. Res., 85, 7383-7398, 1980.

Cho, H., Shepson, P. B., Barrie, L. A., Cowin, J. P., and Zaveri, R.: NMR investigation of the quasi-brine layer in ice/brine mixtures, J. Phys. Chem. B, 106, 11226-11232, 2002.

Clyne, M. and Cruse, H.: Atomic resonance fluorescence spectrometry for the rate constants of rapid bimolecular reactions. Part 2. Reactions $\mathrm{Cl}+\mathrm{BrCl}, \mathrm{Cl}+\mathrm{Br}_{2}, \mathrm{Cl}+\mathrm{ICl}, \mathrm{Br}+\mathrm{IBr}, \mathrm{Br}+\mathrm{ICl}$, J. Chem. Soc., Faraday Trans. 2, 68, 1377-1387, 1972.

Custard, K. D., Thompson, C. R., Pratt, K. A., Shepson, P. B., Liao, J., Huey, L. G., Orlando, J. J., Weinheimer, A. J., Apel, E., Hall, S. R., Flocke, F., Mauldin, L., Hornbrook, R. S., Pöhler, D., General, S., Zielcke, J., Simpson, W. R., Platt, U., Fried, A., Weibring, P., Sive, B. C., Ullmann, K., Cantrell, C., Knapp, D. J., and Montzka, D. D.: The $\mathrm{NO}_{x}$ dependence of bromine chemistry in the Arctic atmospheric boundary layer, Atmos. Chem. Phys. Discuss., 15, 8329-8360, doi:10.5194/acpd-15-8329-2015, 2015.

Davis, D., Crawford, J., Liu, S., McKeen, S., Bandy, A., Thornton, D., Rowland, F., and Blake, D.: Potential impact of iodine on tropospheric levels of ozone and other critical oxidants, J. Geophys. Res., 101, 2135-2147, 1996.

DeMore, W. B., Sander, S., Golden, D., Hampson, R., Kurylo, M., Howard, C., Ravishankara, A., Kolb, C., Molina, M., and Jet Propulsion Lab., C. I. O. T., Pasadena.: Chemical kinetics and photochemical data for use in stratospheric modeling, Jet Propulsion Lab., California Inst. of Tech., Pasadena, CA, USA, 1997.

Donahue, N. M., Anderson, J. G., and Demerjian, K. L.: New rate constants for ten $\mathrm{OH}$ alkane reactions from 300 to $400 \mathrm{~K}$ : an assessment of accuracy, J. Phys. Chem. A, 102, 3121-3126, 1998.

Dunlea, E. J. and Ravishankara, A.: Kinetic studies of the reactions of $\mathrm{O}\left({ }^{1} D\right)$ with several atmospheric molecules, Phys. Chem. Chem. Phys., 6, 2152-2161, 2004.

Eberhard, J. and Howard, C. J.: Temperature-dependent kinetics studies of the reactions of $\mathrm{C}_{2} \mathrm{H}_{5} \mathrm{O}_{2}$ and $n-\mathrm{C}_{3} \mathrm{H}_{7} \mathrm{O}_{2}$ radicals with NO, Int. J. Chem. Kinet., 28, 731-740, 1996.

Eberhard, J., Villalta, P. W., and Howard, C. J.: Reaction of isopropyl peroxy radicals with NO over the temperature range 201401 K, J. Phys. Chem., 100, 993-997, 1996.

Fickert, S., Adams, J. W., and Crowley, J. N.: Activation of $\mathrm{Br}_{2}$ and $\mathrm{BrCl}$ via uptake of $\mathrm{HOBr}$ onto aqueous salt solutions, J. Geophys. Res., 104, 23719-23727, doi:10.1029/1999JD900359, 1999.

Fisher, J. A., Jacob, D. J., Wang, Q., Bahreini, R., Carouge, C. C., Cubison, M. J., Dibb, J. E., Diehl, T., Jimenez, J. L., and Leibensperger, E. M.: Sources, distribution, and acidity of sulfate-ammonium aerosol in the Arctic in winter-spring, Atmos. 
Environ., 45, 7301-7318, doi:10.1016/j.atmosenv.2011.08.030, 2011.

Fried, A., Sewell, S., Henry, B., Wert, B. P., and Gilpin, T.: Tunable diode laser absorption spectrometer for ground-based measurements of formaldehyde, J. Geophys. Res., 102, 6253-6266, 1997.

Frieß, U., Deutschmann, T., Gilfedder, B. S., Weller, R., and Platt, U.: Iodine monoxide in the Antarctic snowpack, Atmos. Chem. Phys., 10, 2439-2456, doi:10.5194/acp-10-2439-2010, 2010.

Ganske, J., Berko, H., and Finlayson-Pitts, B.: Absorption Cross Sections for Gaseous $\mathrm{ClNO}_{2}$ and $\mathrm{Cl}_{2}$ at $298 \mathrm{~K}$ : Potential Organic Oxidant Source in the Marine Troposphere, J. Geophys. Res., 97, 7651-7656, 1992.

Gao, S., Sjostedt, S., Sharma, S., Hall, S., Ullmann, K., and Abbatt, J.: PTR-MS observations of photo-enhanced VOC release from Arctic and mid-latitude snow, J. Geophys. Res., 117, D00R17, doi:10.1029/2011JD017152, 2012.

Garland, J. and Curtis, H.: Emission of iodine from the sea surface in the presence of ozone, J. Geophys. Res., 86, 3183-3186, 1981.

Gomez Martin, J. C., Spietz, P., and Burrows, J. P.: Spectroscopic studies of the $\mathrm{I}_{2} / \mathrm{O}_{3}$ photochemistry Part 1 : Determination of the absolute absorption cross sections of iodine oxides of atmospheric relevance, J. Photochem. Photobio. A, Chemistry, 176, 15-38, 2005.

Gong, S., Walmsley, J., Barrie, L., and Hopper, J.: Mechanisms for surface ozone depletion and recovery during polar sunrise, Atmos. Environ., 31, 969-981, 1997.

Gosselin, M., Levasseur, M., Wheeler, P. A., Horner, R. A., and Booth, B. C.: New measurements of phytoplankton and ice algal production in the Arctic Ocean, Deep Sea Research Part II: Topical Studies in Oceanography, 44, 1623-1644, 1997.

Grannas, A. M., Shepson, P. B., Guimbaud, C., Sumner, A. L., Albert, M., Simpson, W., Domine, F., Boudries, H., Bottenheim, J., and Beine, H. J.: A study of photochemical and physical processes affecting carbonyl compounds in the Arctic atmospheric boundary layer, Atmos. Environ., 36, 2733-2742, 2002.

Grannas, A. M., Jones, A. E., Dibb, J., Ammann, M., Anastasio, C., Beine, H. J., Bergin, M., Bottenheim, J., Boxe, C. S., Carver, G., Chen, G., Crawford, J. H., Dominé, F., Frey, M. M., Guzmán, M. I., Heard, D. E., Helmig, D., Hoffmann, M. R., Honrath, R. E., Huey, L. G., Hutterli, M., Jacobi, H. W., Klán, P., Lefer, B., McConnell, J., Plane, J., Sander, R., Savarino, J., Shepson, P. B., Simpson, W. R., Sodeau, J. R., von Glasow, R., Weller, R., Wolff, E. W., and Zhu, T.: An overview of snow photochemistry: evidence, mechanisms and impacts, Atmos. Chem. Phys., 7, 43294373, doi:10.5194/acp-7-4329-2007, 2007.

Guimbaud, C., Grannas, A. M., Shepson, P. B., Fuentes, J. D., Boudries, H., Bottenheim, J. W., Dominé, F., Houdier, S., Perrier, S., and Biesenthal, T. B.: Snowpack processing of acetaldehyde and acetone in the Arctic atmospheric boundary layer, Atmos. Environ., 36, 2743-2752, 2002.

Halfacre, J. W., Knepp, T. N., Shepson, P. B., Thompson, C. R., Pratt, K. A., Li, B., Peterson, P. K., Walsh, S. J., Simpson, W. R., Matrai, P. A., Bottenheim, J. W., Netcheva, S., Perovich, D. K., and Richter, A.: Temporal and spatial characteristics of ozone depletion events from measurements in the Arctic, Atmos. Chem. Phys., 14, 4875-4894, doi:10.5194/acp-14-4875-2014, 2014.
Hansen, J. C., Li, Y., Li, Z., and Francisco, J. S.: On the mechanism of the $\mathrm{BrO}+\mathrm{HBr}$ reaction, Chem. Phys. Lett., 314, 341$346,1999$.

Harris, S. J. and Kerr, J. A.: Relative rate measurements of some reactions of hydroxyl radicals with alkanes studied under atmospheric conditions, Int. J. Chem. Kinet., 20, 939-955, 1988.

Hausmann, M. and Platt, U.: Spectroscopic measurement of bromine oxide and ozone in the high Arctic during Polar Sunrise Experiment 1992, J. Geophys. Res., 99, 25399-25413, doi:10.1029/94JD01314, 1994.

Helmig, D., Ganzeveld, L., Butler, T., and Oltmans, S. J.: The role of ozone atmosphere-snow gas exchange on polar, boundary-layer tropospheric ozone - a review and sensitivity analysis, Atmos. Chem. Phys., 7, 15-30, doi:10.5194/acp-7-15-2007, 2007.

Helmig, D., Boylan, P., Johnson, B., Oltmans, S., Fairall, C., Staebler, R., Weinheimer, A., Orlando, J., Knapp, D. J., Montzka, D. D., Flocke, F., Frieß, U., Sihler, H., and Shepson, P. B.: Ozone dynamics and snow-atmosphere exchanges during ozone depletion events at Barrow, Alaska, J. Geophys. Res., 117, D20303, doi:10.1029/2012JD017531, 2012.

Hönninger, G.: Halogen Oxide Studies in the Boundary Layer by Multi Axis Differential Optical Absorption Spectroscopy and Active Longpath-DOAS, PhD, University of Heidelberg, Germany, 2002.

Honrath, R., Peterson, M., Guo, S., Dibb, J., Shepson, P., and Campbell, B.: Evidence of NOx production within or upon ice particles in the Greenland snowpack, Geophys. Res. Lett, 26, 695-698, 1999.

Hooshiyar, P. A. and Niki, H.: Rate constants for the gas phase reactions of $\mathrm{Cl}$ atoms with C2-C8 alkanes at $T=296 \pm 2 \mathrm{~K}$, Int. J Chem. Kinet., 27, 1197-1206, 1995.

Huff, A. K. and Abbatt, J. P. D.: Kinetics and product yields in the heterogeneous reactions of $\mathrm{HOBr}$ with ice surfaces containing $\mathrm{NaBr}$ and $\mathrm{NaCl}$, J. Phys. Chem. A, 106, 5279-5287, 2002.

Impey, G., Shepson, P., Hastie, D., Barrie, L., and Anlauf, K.: Measurements of photolyzable chlorine and bromine during the Polar Sunrise Experiment 1995, J. Geophys. Res., 102, 16005-16010, 1997.

Jacob, D. J.: Heterogeneous chemistry and tropospheric ozone, Atmos. Environ., 34, 2131-2159, 2000.

Jacobi, H. W., Kaleschke, L., Richter, A., Rozanov, A., and Burrows, J. P.: Observation of a fast ozone loss in the marginal ice zone of the Arctic Ocean, J. Geophys. Res., 111, D15309, doi:10.1029/2005JD006715, 2006.

Jobson, B., Niki, H., Yokouchi, Y., Bottenheim, J., Hopper, F., and Leaitch, R.: Measurements of C2-C6 hydrocarbons during the Polar Sunrise 1992 Experiment: Evidence for $\mathrm{Cl}$ atom and $\mathrm{Br}$ atom chemistry, J. Geophys. Res, 99, 25355-25368, 1994.

Jones, C. E., Hornsby, K. E., Sommariva, R., Dunk, R. M., von Glasow, R., McFiggans, G., and Carpenter, L. J.: Quantifying the contribution of marine organic gases to atmospheric iodine, Geophys. Res. Lett., 37, L18804, doi:10.1029/2010GL043990, 2010.

Kamboures, M. A., Hansen, J. C., and Francisco, J. S.: A study of the kinetics and mechanisms involved in the atmospheric degradation of bromoform by atomic chlorine, Chem. Phys. Lett., 353, 335-344, 2002.

Keil, A. and Shepson, P.: Chlorine and bromine atom ratios in the springtime Arctic troposphere as determined from measurements 
of halogenated volatile organic compounds, J. Geophys. Res, 111, D17303, doi:10.1029/2006JD007119, 2006.

King, M. D. and Simpson, W. R.: Extinction of UV radiation in Arctic snow at Alert, Canada $\left(82^{\circ} \mathrm{N}\right)$, J. Geophys. Res, 106, 499412, 2001.

Kirchner, F. and Stockwell, W. R.: Effect of peroxy radical reactions on the predicted concentrations of ozone, nitrogenous compounds, and radicals, J. Geophys. Res., 101, 21007-21022, 1996.

Knepp, T. N., Bottenheim, J., Carlsen, M., Carlson, D., Donohoue, D., Friederich, G., Matrai, P. A., Netcheva, S., Perovich, D. K., Santini, R., Shepson, P. B., Simpson, W., Valentic, T., Williams, C., and Wyss, P. J.: Development of an autonomous sea ice tethered buoy for the study of ocean-atmosphere-sea ice-snow pack interactions: the O-buoy, Atmos. Meas. Tech., 3, 249-261, doi:10.5194/amt-3-249-2010, 2010.

Krnavek, L., Simpson, W. R., Carlson, D., Domine, F., Douglas, T. A., and Sturm, M.: The chemical composition of surface snow in the Arctic: Examining marine, terrestrial, and atmospheric influences, Atmos. Environ., 50, 349-359, doi:10.1016/j.atmosenv.2011.11.033, 2011.

Kukui, A., Kirchner, U., Benter, T., and Schindler, R.: A Gaskinetic Investigation of $\mathrm{HOBr}$ Reactions with $\mathrm{CI}(2 \mathrm{P}), \mathrm{O}(3 \mathrm{P})$ and $\mathrm{OH}$ (2II). The Reaction of $\mathrm{BrCl}$ with $\mathrm{OH}$ (2II), Berichte der Bunsengesellschaft für physikalische Chemie, 100, 455-461, 1996.

Lancaster, D. G., Fried, A., Wert, B., Henry, B., and Tittel, F. K.: Difference-frequency-based tunable absorption spectrometer for detection of atmospheric formaldehyde, Appl. Optics, 39, 44364443, 2000.

Le Bras, G. and Platt, U.: A possible mechanism for combined chlorine and bromine catalyzed destruction of tropospheric ozone in the Arctic, Geophys. Res. Lett., 22, 599-602, 1995.

Lehrer, E., Hönninger, G., and Platt, U.: A one dimensional model study of the mechanism of halogen liberation and vertical transport in the polar troposphere, Atmos. Chem. Phys., 4, 24272440, doi:10.5194/acp-4-2427-2004, 2004.

Li, S.-M. and Winchester, J. W.: Resolution of ionic components of late winter Arctic aerosols, Atmos. Environ. 23, 2387-2399, 1989.

Li, X, Rohrer, F., Hofzumahaus, A., Brauers, T., Häseler, R., Bohn, B., Broch, S., Fuchs, H., Gomm, S., Holland, F., Jäger, J., Kaiser, J., Keutsch, F. N., Lohse, I., Lu, K., Tillmann, R., Wegener, R., Wolfe, G. M., Mentel, T. F., Kiendler-Scharr, A., and Wahner, A.: Missing gas-phase source of HONO inferred from zeppelin measurements in the troposphere, Science, 344, 292-296, 2014.

Liao, J., Sihler, H., Huey, L. G., Neumann, J. A., Tanner, D. J., Friess, U., Platt, U., Flocke, F. M., Orlando, J. J., Shepson, P. B., Beine, H. J., Weinheimer, A. J., Sjostedt, S. J., Nowak, J. B., Knapp, D. J., Staebler, R. M., Zheng, W., Sander, R., Hall, S. R., and Ullmann, K.: A comparison of Arctic BrO measurements by chemical ionization mass spectrometry and long-path differential optical absorption spectroscopy, J. Geophys. Res., 116, D00R02, doi:10.1029/2010JD014788, 2011.

Liao, J., Huey, L., Tanner, D., Flocke, F., Orlando, J., Neuman, J., Nowak, J., Weinheimer, A., Hall, S., Smith, J., Fried, A., Staebler, R., Wang, Y., Koo, J.-H., Cantrell, C., Weibring, P., Walega, J., Knapp, D., Shepson, P., and Stephens, C.: Observations of inorganic bromine $\left(\mathrm{HOBr}, \mathrm{BrO}\right.$, and $\left.\mathrm{Br}_{2}\right)$ speciation at Barrow, Alaska, in spring 2009, J. Geophys. Res., 117, D00R16, doi:10.1029/2011JD016641, 2012.
Liao, J., Huey, L. G., Liu, Z., Tanner, D. J., Cantrell, C. A., Orlando, J. J., Flocke, F. M., Shepson, P. B., Weinheimer, A. J., Hall, S. R., Ullmann, K., Beine, H. J., Wang, Y., Ingall, E. D., Stephens, C. R., Hornbrook, R. S., Apel, E. C., Riemer, D., Fried, A., Mauldin III, R. L., Smith, J. N., Staebler, R. M., Neuman, J. A., and Nowak, J. B.: High levels of molecular chlorine in the Arctic atmosphere, Nat. Geosci., 7, 91-94, doi:10.1038/ngeo2046, 2014.

Lightfoot, P., Cox, R., Crowley, J., Destriau, M., Hayman, G., Jenkin, M., Moortgat, G., and Zabel, F.: Organic peroxy radicals: kinetics, spectroscopy and tropospheric chemistry, Atmos. Environ., 26, 1805-1961, 1992.

Lizotte, M. P.: The contributions of sea ice algae to Antarctic marine primary production, Am. Zool., 41, 57-73, 2001.

Lurmann, F. W., Lloyd, A. C., and Atkinson, R.: A chemical mechanism for use in long-range transport/acid deposition computer modeling, J. Geophys. Res., 91, 10905-10936, 1986.

Madronich, S. and Flocke, S.: The role of solar radiation in atmospheric chemistry, in: Handbook of Environmental Chemistry, Springer-Verlag, Berlin, Heidelberg, Germany, 1-26, 1999.

Mahajan, A., Shaw, M., Oetjen, H., Hornsby, K., Carpenter, L., Kaleschke, L., Tian-Kunze, X., Lee, J., Moller, S., Edwards, P., Commane, R., Ingham, T., Heard, D. E., and Plane, J. M. C.: Evidence of reactive iodine chemistry in the Arctic boundary layer, J. Geophys. Res., 115, D20303, doi:10.1029/2009JD013665, 2010.

Mallard, W. G., Westley, F., Herron, J. T., Hampson, R. F., and Frizzel, D. H.: NIST Chemical Kinetics Database: Version 5.0, National Institute of Standards and Technology, Gaithersburg, MD, USA, 1993.

Martinez, M., Arnold, T., and Perner, D.: The role of bromine and chlorine chemistry for arctic ozone depletion events in NyÅlesund and comparison with model calculations, Ann. Geophys., 17, 941-956, 1999, http://www.ann-geophys.net/17/941/1999/.

Martino, M., Mills, G. P., Woeltjen, J., and Liss, P. S.: A new source of volatile organoiodine compounds in surface seawater, Geophys. Res.Lett., 36, L01609, doi:10.1029/2008GL036334, 2009.

McFiggans, G., Plane, J. M. C., Allan, B. J., Carpenter, L. J., Coe, H., and O'Dowd, C.: A modeling study of iodine chemistry in the marine boundary layer, J. Geophys. Res., 105, 14371-14385, 2000.

McFiggans, G., Cox, R. A., Mössinger, J. C., Allan, B. J., and Plane, J. M. C.: Active chlorine release from marine aerosols: Roles for reactive iodine and nitrogen species, J. Geophys. Res, 107, 4271, doi:10.1029/2001JD000383, 2002.

Michalowski, B. A., Francisco, J. S., Li, S. M., Barrie, L. A., Bottenheim, J. W., and Shepson, P. B.: A computer model study of multiphase chemistry in the Arctic boundary layer during polar sunrise, J. Geophys. Res., 105, 15131-15145, doi:10.1029/2000JD900004, 2000.

Nghiem, S. V., Rigor, I. G., Richter, A., Burrows, J. P., Shepson, P. B., Bottenheim, J. W., Barber, D. G., Steffen, A., Latonas, J. R., and Wang, F.: Field and satellite observations of the formation and distribution of Arctic atmospheric bromine above a rejuvenated sea ice cover, J. Geophys. Res., 117, D00S05, doi:10.1029/2011JD016268, 2012.

Nicovich, J. and Wine, P.: Kinetics of the reactions of $\mathrm{O}\left({ }^{3} \mathrm{P}\right)$ and $\mathrm{Cl}$ $\left({ }^{2} \mathrm{P}\right)$ with $\mathrm{HBr}$ and $\mathrm{Br}_{2}$, Int. J. Chem. Kinet., 22, 379-397, 1990.

Oltmans, S. J. and Komhyr, W. D.: Surface ozone distributions and variations from 1973-1984 measurements at the NOAA geo- 
physical monitoring for climatic change baseline observatories, J. Geophys. Res., 91, 5229-5236, 1986.

Oltmans, S. J., Johnson, B. J., and Harris, J. M.: Springtime boundary layer ozone depletion at Barrow, Alaska: Meteorological influence, year-to-year variation, and long-term change, J. Geophys. Res., 117, D00R18, doi:10.1029/2011jd016889, 2012.

Orlando, J. J. and Burkholder, J. B.: Identification of BrONO as the major product in the gas-phase reaction of $\mathrm{Br}$ with $\mathrm{NO}_{2}$, J. Phys. Chem. A, 104, 2048-2053, 2000.

Orlando, J. J. and Tyndall, G. S.: Rate Coefficients for the Thermal Decomposition of $\mathrm{BrONO}_{2}$ and the Heat of Formation of BrONO$_{2}$, J. Phys. Chem., 100, 19398-19405, 1997.

Oum, K., Lakin, M., DeHaan, D., Brauers, T., and FinlaysonPitts, B.: Formation of molecular chlorine from the photolysis of ozone and aqueous sea-salt particles, Science, 279, 74-76, doi:10.1126/science.279.5347.74, 1998a.

Oum, K., Lakin, M., and Finlayson-Pitts, B.: Bromine activation in the troposphere by the dark reaction of $\mathrm{O}_{3}$ with seawater ice, Geophys. Res. Lett., 25, 3923-3926, 1998b.

Piot, M. and von Glasow, R.: The potential importance of frost flowers, recycling on snow, and open leads for ozone depletion events, Atmos. Chem. Phys., 8, 2437-2467, doi:10.5194/acp-82437-2008, 2008.

Piot, M. and von Glasow, R.: Modelling the multiphase near-surface chemistry related to ozone depletions in polar spring, J. Atmos. Chem., 64, 77-105, 2009.

Platt, U. and Hönninger, G.: The role of halogen species in the troposphere, Chemosphere, 52, 325-338, 2003.

Platt, U. and Janssen, C.: Observation and role of the free radicals $\mathrm{NO}_{3}, \mathrm{ClO}, \mathrm{BrO}$ and $\mathrm{IO}$ in the troposphere, Faraday Discuss., 100, 175-198, 1995.

Pöhler, D., Vogel, L., Frieß, U., and Platt, U.: Observation of halogen species in the Amundsen Gulf, Arctic, by active long-path differential optical absorption spectroscopy, P. Natl Acad. Sci., 107, 6582-6587, doi:10.1073/pnas.0912231107, 2010.

Pratt, K. A., Custard, K. D., Shepson, P. B., Douglas, T. A., Pöhler, D., General, S., Zielcke, J., Simpson, W. R., Platt, U., and Tanner, D. J.: Photochemical production of molecular bromine in Arctic surface snowpacks, Nat. Geosci., 6, 351-356, 2013.

Raso, A. R. W., Custard, K. D., Pratt, K. A., Tanner, D. J., Huey, L. G., and Shepson, P. B.: Active molecular iodine snowpack photochemistry in the Arctic, J. Geophys. Res., submitted, 2015.

Ravishankara, A. R., Smith, G. J., and Davis, D. D.: A kinetics study of the reaction of $\mathrm{Cl}$ with $\mathrm{NO}_{2}$, Int. J. Chem. Kinet., 20, 811-814, doi:10.1002/kin.550201005, 1988.

Ridley, B., Grahek F., and Walega, J.: A small high-sensitivity, medium-response ozone detector suitable for measurements from light aircraft, J. Atmos. Oceanic Technol., 9, 142-148, 1992.

Roberts, J. M., Osthoff, H. D., Brown, S. S., and Ravishankara, A.: $\mathrm{N}_{2} \mathrm{O}_{5}$ oxidizes chloride to $\mathrm{Cl}_{2}$ in acidic atmospheric aerosol, Science, 321, 1059-1059, 2008.

Rowley, D. M., Bloss, W. J., Cox, R. A., and Jones, R. L.: Kinetics and products of the $\mathrm{IO}+\mathrm{BrO}$ reaction, J. Phys. Chem. A., 105, 7855-7864, doi:10.1021/jp004494y, 2001.

Rudolph, J., Fu, B., Thompson, A., Anlauf, K., and Bottenheim, J.: Halogen atom concentrations in the Arctic troposphere derived from hydrocarbon measurements: Impact on the budget of formaldehyde, Geophys. Res. Lett., 26, 2941-2944, 1999.
Russo, R. S., Zhou, Y., White, M. L., Mao, H., Talbot, R., and Sive, B. C.: Multi-year (2004-2008) record of nonmethane hydrocarbons and halocarbons in New England: seasonal variations and regional sources, Atmos. Chem. Phys., 10, 4909-4929, doi:10.5194/acp-10-4909-2010, 2010.

Ryerson, T. B., Williams, E. J., and Fehsenfeld, F. C.: An efficient photolysis system for fast-response $\mathrm{NO}_{2}$ measurements, J. Geophys. Res., 105, 26447-26461, 2000.

Saiz-Lopez, A. and Plane, J. M. C.: Novel iodine chemistry in the marine boundary layer, Geophys. Res. Lett., 31, L04112, doi:10.1029/2003GL019215, 2004.

Saiz-Lopez, A., Chance, K., Liu, X., and Kurosu, T. P.: First observations of iodine oxide from space, Geophys. Res. Lett., 34, L12812, doi:10.1029/2007GL030111, 2007a.

Saiz-Lopez, A., Mahajan, A. S., Salmon, R. A., Bauguitte, S. J. B., Jones, A. E., Roscoe, H. K., and Plane, J.: Boundary layer halogens in coastal Antarctica, Science, 317, 348-351, doi:10.1126/science.1141408, 2007b.

Saiz-Lopez, A., Plane, J. M. C., Mahajan, A. S., Anderson, P. S., Bauguitte, S. J.-B., Jones, A. E., Roscoe, H. K., Salmon, R. A., Bloss, W. J., Lee, J. D., and Heard, D. E.: On the vertical distribution of boundary layer halogens over coastal Antarctica: implications for $\mathrm{O}_{3}, \mathrm{HO}_{x}, \mathrm{NO}_{x}$ and the $\mathrm{Hg}$ lifetime, Atmos. Chem. Phys., 8, 887-900, doi:10.5194/acp-8-887-2008, 2008.

Saiz-Lopez, A., Plane, J. M. C., Baker, A. R., Carpenter, L. J., von Glasow, R., Gomez Martin, J. C., McFiggans, G., and Saunders, R. W.: Atmospheric Chemistry of Iodine, Chem. Rev., 112, 1773-1804, doi:10.1021/cr200029u, 2011.

Sander, R. and Crutzen, P. J.: Model study indicating halogen activation and ozone destruction in polluted air masses transported to the sea, J. Geophys. Res., 101, 9121-9138, 1996.

Sander, R., Vogt, R., Harris, G. W., and Crutzen, P. J.: Modelling the chemistry of ozone, halogen compounds, and hydrocarbons in the arctic troposphere during spring, Tellus B, 49, 522-532, 1997.

Sander, S. P., Golden, D., Kurylo, M., Moortgat, G., Wine, P., Ravishankara, A., Kolb, C., Molina, M., Finlayson-Pitts, B., and Huie, R.: Chemical kinetics and photochemical data for use in atmospheric studies evaluation number 15, JPL Publication 062, NASA Jet Propulsion Laboratory, Pasadena, California, USA, 2006.

Saunders, R. W. and Plane, J. M. C.: Formation pathways and composition of iodine oxide ultra-fine particles, Environ. Chem., 2, 299-303, 2006.

Schönhardt, A., Richter, A., Wittrock, F., Kirk, H., Oetjen, H., Roscoe, H. K., and Burrows, J. P.: Observations of iodine monoxide columns from satellite, Atmos. Chem. Phys., 8, 637-653, doi:10.5194/acp-8-637-2008, 2008.

Schweitzer, F., Mirabel, P., and George, C.: Multiphase chemistry of $\mathrm{N}_{2} \mathrm{O}_{5}, \mathrm{ClNO}_{2}$, and $\mathrm{BrNO}_{2}$, J. Phys. Chem. A, 102, 3942-3952, 1998.

Shepson, P. B., Sirju, A.-P., Hopper, J. F., Barrie, L. A., Young, V., Niki, H., and Dryfhout, H.: Sources and sinks of carbonyl compounds in the Arctic Ocean boundary layer: Polar Ice Floe Experiment, J. Geophys. Res., 101, 21081-21089, 1996.

Simpson, W. R., von Glasow, R., Riedel, K., Anderson, P., Ariya, P., Bottenheim, J., Burrows, J., Carpenter, L. J., Frieß, U., Goodsite, M. E., Heard, D., Hutterli, M., Jacobi, H.-W., Kaleschke, L., Neff, B., Plane, J., Platt, U., Richter, A., Roscoe, H., Sander, 
R., Shepson, P., Sodeau, J., Steffen, A., Wagner, T., and Wolff, E.: Halogens and their role in polar boundary-layer ozone depletion, Atmos. Chem. Phys., 7, 4375-4418, doi:10.5194/acp-74375-2007, 2007.

Sirois, A. and Barrie, L. A.: Arctic lower tropospheric aerosol trends and composition at Alert, Canada: 1980-1995, J. Geophys. Res., 104, 11599-11618, 1999

Sommariva, R., Bloss, W., and von Glasow, R.: Uncertainties in gasphase atmospheric iodine chemistry, Atmos. Environ., 57, 219232, 2012.

Stephens, C. R., Shepson, P. B., Steffen, A., Bottenheim, J. W., Liao, J., Huey, L. G., Apel, E., Weinheimer, A., Hall, S. R., and Cantrell, C.: The relative importance of chlorine and bromine radicals in the oxidation of atmospheric mercury at Barrow, Alaska, J. Geophys. Res., 117, D00R11, doi:10.1029/2011JD016649, 2012.

Sturges, W. and Barrie, L.: Chlorine, bromine and iodine in Arctic aerosols, Atmos. Environ., 22, 1179-1194, 1988.

Sumner, A., Shepson, P., Grannas, A., Bottenheim, J., Anlauf, K., Worthy, D., Schroeder, W., Steffen, A., Domine, F., and Perrier, S.: Atmospheric chemistry of formaldehyde in the Arctic troposphere at Polar Sunrise, and the influence of the snowpack, Atmos. Environ., 36, 2553-2562, 2002.

Sumner, A. L. and Shepson, P. B.: Snowpack production of formaldehyde and its effect on the Arctic troposphere, Nature, 398, 230-233, 1999.

Tackett, P., Cavender, A., Keil, A., Shepson, P., Bottenheim, J., Morin, S., Deary, J., Steffen, A., and Doerge, C.: A Study of the vertical scale of halogen chemistry in the Arctic troposphere during polar sunrise at Barrow, AK, J. Geophys. Res, 112, D07306, doi:10.1029/2006JD007785, 2007.

Tang, T. and McConnell, J.: Autocatalytic release of bromine from Arctic snow pack during polar sunrise, Geophys. Res. Lett., 23, 2633-2636, 1996.

Thomas, J. L., Stutz, J., Lefer, B., Huey, L. G., Toyota, K., Dibb, J. E., and von Glasow, R.: Modeling chemistry in and above snow at Summit, Greenland - Part 1: Model description and results, Atmos. Chem. Phys., 11, 4899-4914, doi:10.5194/acp-11-48992011, 2011.

Thomas, J. L., Dibb, J. E., Huey, L. G., Liao, J., Tanner, D., Lefer, B., von Glasow, R., and Stutz, J.: Modeling chemistry in and above snow at Summit, Greenland - Part 2: Impact of snowpack chemistry on the oxidation capacity of the boundary layer, Atmos. Chem. Phys., 12, 6537-6554, doi:10.5194/acp-12-65372012, 2012.

Thompson, C. R., Shepson, P. B., Liao, J., Huey, L. G., Cantrell, C., Flocke, F., and Orlando, J.: Bromine atom production and chain propagation during springtime Arctic ozone depletion events in Barrow, Alaska, in prep., 2015.

Troy, R. C., Kelley, M. D., Nagy, J. C., and Margerum, D. W.: Non-metal redox kinetics: Iodine monobromide reaction with iodide ion and the hydrolysis of IBr, Inorg. Chem., 30, 4838-4845, 1991.

Tsalkani, N., Mellouki, A., Poulet, G., Toupance, G., and Bras, G.: Rate constant measurement for the reactions of $\mathrm{OH}$ and $\mathrm{Cl}$ with peroxyacetyl nitrate at $298 \mathrm{~K}$, J. Atmos. Chem., 7, 409-419, 1988.
Tuckermann, M., Ackermann, R., Gölz, C., Lorenzen-Schmidt, H., Senne, T., Stutz, J., Trost, B., Unold, W., and Platt, U.: DOASobservation of halogen radical-catalysed arctic boundary layer ozone destruction during the ARCTOC-campaigns 1995 and 1996 in Ny-Ålesund, Spitsbergen, Tellus B, 49, 533-555, 1997.

Turnipseed, A. A., Gilles, M. K., Burkholder, J. B., and Ravishankara, A. R.: Kinetics of the IO radical. 1. Reaction of Io with ClO, J. Phys. Chem. A, 101, 5517-5525, doi:10.1021/jp970914g, 1997.

Tyndall, G., Orlando, J., Wallington, T., Dill, M., and Kaiser, E.: Kinetics and mechanisms of the reactions of chlorine atoms with ethane, propane, and n-butane, Int. J. Chem. Kinet., 29, 43-55, 1997.

Vakhtin, A. B., Murphy, J. E., and Leone, S. R.: Low-temperature kinetics of reactions of $\mathrm{OH}$ radical with ethene, propene, and 1butene, J. Phys. Chem. A, 107, 10055-10062, 2003.

Villena, G., Wiesen, P., Cantrell, C. A., Flocke, F., Fried, A., Hall, S. R., Hornbrook, R. S., Knapp, D., Kosciuch, E., Mauldin III, R. L., McGrath, J. A., Montzka, D., Richter, D., Ullmann, K., Walega, J., Weibring, P., Weinheimer, A., Staebler, R. M., Liao, J., Huey, L. G., and Kleffmann, J.: Nitrous acid (HONO) during polar spring in Barrow, Alaska: a net source of $\mathrm{OH}$ radicals?, J. Geophys. Res., 116, D00R07, doi:10.1029/2011JD016643, 2011.

Vogt, R.: Iodine compounds in the atmosphere, in: Reactive halogen compounds in the atmosphere, The Handbook of Environmental Chemistry, 4E, Springer, Berlin, Heidelberg, Germany, 113-128, doi:10.1007/10628761, 1999.

Vogt, R., Crutzen, P. J., and Sander, R.: A mechanism for halogen release from sea-salt aerosol in the remote marine boundary layer, Nature, 383, 327-330, doi:10.1038/383327a0, 1996.

Wallington, T. J., Skewes, L. M., Siegl, W. O., Wu, C. H., and Japar, S. M.: Gas phase reaction of $\mathrm{Cl}$ atoms with a series of oxygenated organic species at 295 K, Int. J. Chem. Kinet., 20, 867-875, 1988.

Wallington, T. J., Skewes, L. M., Siegl, W. O., and Japar, S. M.: A relative rate study of the reaction of bromine atoms with a variety of organic compounds at $295 \mathrm{~K}$, Int. J. Chem. Kinet., 21, 1069 1076, 1989.

Wang, T. X. and Margerum, D. W.: Kinetics of reversible chlorine hydrolysis: Temperature dependence and general-acid/baseassisted mechanisms, Inorg. Chem., 33, 1050-1055, 1994.

Wang, T. X., Kelley, M. D., Cooper, J. N., Beckwith, R. C., and Margerum, D. W.: Equilibrium, kinetic, and UV-spectral characteristics of aqueous bromine chloride, bromine, and chlorine species, Inorg. Chem., 33, 5872-5878, 1994.

Wang, Y. L., Nagy, J. C., and Margerum, D. W.: Kinetics of hydrolysis of iodine monochloride measured by the pulsed-acceleratedflow method, J. Amer. Chem. Soc., 111, 7838-7844, 1989.

Wine, P., Wells, J., and Nicovich, J.: Kinetics of the reactions of atomic fluorine (2P) and atomic chlorine (2P) with nitric acid, J. Phys. Chem., 92, 2223-2228, 1988.

Zhou, X., Beinel, H. J., Honrath, R. E., Fuentes, J. D., Simpson, W., Shepson, P. B., and Bottenheim, J. W.: Snowpack Photochemical Production of HONO: a major source of $\mathrm{OH}$ in the Arctic boundary layer in springtime, Geophys. Res. Lett., 28, 40874090, 2001. 